\title{
Determination of Carbohydrate-Deficient Transferrin in Human Serum by Capillary Zone Electrophoresis
}

\author{
Inauguraldissertation \\ zur \\ Erlangung der Würde eines Doktors der Philosophie \\ vorgelegt der \\ Philosophisch-Naturwissenschaftlichen Fakultät \\ der Universität Basel \\ von
}

Christian Lanz

aus Roggwil BE

Bern, 2004 
Genehmigt von der Philosophisch-Naturwissenschaftlichen Fakultät auf Antrag von

Prof. Dr. phil. nat. Wolfgang Thormann als Dissertationsleiter

Prof. Dr. pharm. Dr. med. Stephan Krähenbühl als Fakultätsverantwortlicher Prof. Dr. med. Jürgen Drewe als Korreferent

Basel, 4. Mai 2004

Prof. Dr. phil. Marcel Tanner 
Dedicated to my parents and my brother Matthias 


\section{Acknowledgements}

I would like to express my sincerest thanks to Prof. Dr. Wolfgang Thormann from the Department of Clinical Pharmacology of the University of Bern for the opportunity of working on this fascinating project in the field of instrumental analysis and clinical chemistry. I do highly appreciate his support as well as the pleasant and friendly atmosphere in his group. I also wish to address my thanks to all my fellow researchers. I should like to mention in particular my appreciation of Martina Kuhn's readiness to share her vast knowledge in the field of clinical chemistry - an invaluable contribution to my work. Furthermore, I wish to thank Dr. Francine Prost, Regula Theurillat, Jitka Caslavska, Andrea Baldacci, Dr. Michael Breadmore, Dr. Anita B. Wey and Andreas Schmid for their cooperativeness and for the countless inspiring discussions; the nurses of the hepatology outpatient clinic for their assistance in obtaining blood samples; Ursula Wanzenried and Jeannette Schiess for their warm reception at the laboratory; Prof. Dr. Franco Tagliaro, Dr. Beat Burckhardt, Dr. Stefan Russmann and Dr. Ulrich Marti for the numerous stimulating discussions. I too wish to express my gratitude to Prof. Dr. Dr. Stephan Krähenbühl from the Institute of Clinical Pharmacology and Toxicology of the University of Basel for supporting my effort to hand in the dissertation at the University of Basel as well as his constructive suggestions regarding the project. Also, I would like to thank Prof. Dr. Jürgen Drewe for accepting to issue the recommendation letter to the faculty of Natural Sciences. Moreover, I wish to thank my parents for their assistance and helpfulness and my brother, Matthias, for the numerous stimulating conversations.

This work was funded by grants from the Liver Foundation, Bern, Switzerland and the Swiss National Science Foundation. 


\section{Table of Contents}

Abbreviations $\quad$ V

A. Introduction 1

A.1. Alcohol Abuse and Carbohydrate-Deficient Transferrin 1

A.2. Capillary Electrophoresis 2

A.3. Goals of the Dissertation 3

B. Results and Discussion $\quad 5$

B.1. Evaluation and optimization of capillary zone electrophoresis with different dynamic coatings for the determination of carbohydrate-deficient transferrin in human serum (J. Chromatogr. A, 979 (2002) 43-57)

B.2. Capillary zone electrophoresis with a dynamic double coating for analysis of carbohydrate-deficient transferrin in human serum: precision performance and pattern recognition (J. Chromatogr. A, 1013 (2003) 131-147)

B.3. Capillary zone electrophoresis with a dynamic double coating for analysis of carbohydrate-deficient transferrin in human serum: impact of resolution between disialo- and trisialotransferrin on reference limits (Electrophoresis, 24 (2003) 4272-4281)

B.4. Improved capillary electrophoresis method for the determaintaion of carbohydrate-deficient transferrin in patient sera (Electrophoresis, 25 (2004) 2309-2318)

D. References 


\section{Abbreviations}

Alb

ALT

AST

CDT

CE

CIEF

CRP

CZE

DAB

EDTA

EO

EOF

GC

GGT

$\mathrm{HCl}$

HPLC

I.D.

IFCC

mAU

$\mathrm{MCV}$

$\mathrm{m}_{\mathrm{eo}}$

$\mathrm{NaCl}$

$\mathrm{NaOH}$

O.D.

pI

psi

$\mathrm{r}$

ROC

Rs

RSD

SD

SPE

Tf

$\mathrm{U}$

UV
Albumin

Alanine aminotransferase

Aspartate aminotransferase

Carbohydrate-deficient transferrin

Capillary electrophoresis

Capillary isoelectric focusing

C-reactive protein

Capillary zone electrophoresis

1,4-diaminobutane

Ethylenediamine tetra-acetic acid

Electroosmosis

Electroosmotic flow

Gas chromatography

$\gamma$-glutamyltransferase

Hydrochloric acid

High-performance liquid chromatography

Inner diameter

International federation of clinical chemistry

Milli absorbance unit

Erythrocyte mean corpuscular volume

Electroosmotic mobility

Sodium chloride

Sodium hydroxide

Outer diameter

Isoelectric point

pound per square inch

Correlation coefficient

Receiver operating characteristic

Resolution

Relative standard deviation

Standard deviation

Serum protein electrophoresis

Transferrin

Unit

Ultraviolet 


\section{A. Introduction}

\section{A.1. Alcohol Abuse and Carbohydrate-Deficient Transferrin}

Alcohol abuse and alcoholism are two widespread problems in practically all ethnic groups with major medical, social, economic and legal consequences for individuals and societies [1-4]. In 1996, $27 \%$ of men and $14 \%$ of women in Great Britain were estimated to drink more than the recommended safe limits of $168 \mathrm{~g}$ and $112 \mathrm{~g}$ pure ethanol per week for men and women, respectively. $7.5 \%$ of men and $2.1 \%$ of women were considered to be alcohol dependent [3]. In 1981, $9 \%$ of the male population and $0.5 \%$ of the females in Switzerland between 15 and 74 years of age stated to drink more than $80 \mathrm{~g}$ pure ethanol daily [5]. In 1998, the overall costs associated with alcohol consumption in Switzerland were estimated at 6.5 billion Swiss francs $[6,7]$. Because of the known ability of alcoholics to hide their addiction for a long time period and because of the importance of an early diagnosis of alcohol abuse, the search for endogenous markers for identifying high risk drinking has attracted great interest for many years. Most of the markers currently used, like alanine aminotransferase, aspartate aminotransferases, erythrocyte mean corpuscular volume, and $\gamma$-glutamyltransferase, suffer from two major drawbacks. They are either indicators of diseases in a particular organ without sufficient relation to a specific etiology, or they lack sensitivity of detecting alcohol abuse before organ damage has occurred [8]. With the description of carbohydrate-deficient transferrin (CDT) by Stibler et al. [9-11], a potent and specific endogenous marker for the detection of chronic excessive alcohol intake and relapse drinking was introduced.

CDT encompasses isoforms of the iron transporting glycoprotein transferrin (Tf, a protein with two iron binding sites) that lack one or both of the carbohydrate side chains. The two N-glycans of the Tf molecule are composed of neutral sugars and zero up to eight terminal, negatively charged sialic acid residues. Thus $\mathrm{Tf}$ isoforms differ in charge and $\mathrm{pI}$ values. CDT includes the $\mathrm{Tf}$ isoforms with zero (asialo-Tf), one (monosialo-Tf) and two (disialo-Tf) sialic acid residues. CDT is considered to be the most specific and reliable indicator of a chronic, excessive alcohol intake. However, the pathophysiologic and biochemical mechanisms responsible for the CDT elevation upon chronic alcohol intake are still not known [12-17]. 
Various analytical approaches have been developed for the qualitative and quantitative determination of CDT. Most of them are suffering from some major drawbacks. Originally, Tf isoform analysis was performed by isoelectric focusing on gels and, due to the high resolution achieved, this principle remains the reference method for qualitative $\mathrm{Tf}$ investigations. However, quantitation of $\mathrm{Tf}$ isoforms by gel isoelectric focusing is difficult to perform and too complex for routine purposes $[1,2,8$ 10,17]. The most commonly used principle for the quantitative analysis of CDT, introduced by Stibler et al. $[18,19]$, is based on the immunochemical determination of CDT. No CDT specific antibodies are available, thus requiring the separation of CDT and non-CDT Tf isoforms. This separation, achieved by anion exchange chromatography on minicolumns, is crucial, difficult and thereby prone to analytical inaccuracy $[2,17,20]$. Jeppsson et al. developed and optimized a high-performance liquid chromatography (HPLC) method which enabled the separation and quantitation of the Tf isoforms. Time consuming sample preparation and low sample throughput are the major disadvantages of this chromatographic approach [21-23].

\section{A.2. Capillary Electrophoresis}

Capillary electrophoresis (CE) is a relatively new separation technique compared to the well known chromatographic procedures, such as HPLC and gas chromatography (GC). Over the past two decades, it has become a powerful analytical tool for the separation of a large variety of compounds, including macromolecules like proteins. In capillary zone electrophoresis (CZE), charged analytes are separated in the electric field according to differences in electrophoretic mobilities [24,25]. Because of its high resolution power, considerable interest has been directed to the analysis of peptides and proteins [26,27], including the analysis of serum proteins, urinary proteins and hemoglobins in clinical laboratories [24]. The main obstacle to overcome is the strong tendency of the highly charged polymers to adsorb to the inner surface of fused silica capillaries, resulting in insufficient separation of the proteins and poor reproducibility. Several strategies have been adopted to reduce the interaction of the proteins with the capillary walls. Simple protocols consisted in the employment of electrolyte solutions at extreme $\mathrm{pH}$ values, suppressing the dissociation of the silanol groups of the capillary surface in an acidic medium, or inducing negative charged surfaces for both, the capillary wall and the proteins, in an alkaline environment. Other strategies are based on the use of buffers of high ionic strength. Alternatively, permanent capillary coatings were employed to selectively modify the surface of the capillary similarly to the derivatized capillaries used in GC. The most promising approach, however, appeared to 
be the introduction of dynamic capillary coatings or polyelectrolyte multilayers for minimizing or abolishing the direct interaction of the proteins with the fused-silica capillaries [28-36].

\section{A.3. Goals of the Dissertation}

The overall goal of this thesis was to develop and evaluate a CZE-based method for the separation and quantitation of CDT in human serum with the ultimate goal of introduction of the assay into routine analysis. To achieve these aims, several aspects had to be considered. First, a procedure minimizing the adsorption of serum proteins to the inner surface of the fused-silica capillary had to be developed. Because of the generally poor long-term performance characteristics of permanently coated capillaries, the investigation of dynamic capillary coatings formed by buffer additives was undertaken. For the determination of CDT in serum, this principle was first investigated by Landers et al. $[37,38]$ and Tagliaro et al. $[39,40]$, whose publications served as the basis for the first project of this dissertation. The goals of this study was to investigate the suitability of two amine-based coatings (1,4-diaminobutane (DAB) and N,N'-bis(3aminopropyl)1,4-butanediamine (spermine)) and the use of commercial reagents producing a dynamic double coating (CEofix-CDT kit, Analis, Namur, Belgium). In the latter approach, a bilayer is formed by noncovalent adsorption. The fused-silica capillary is first rinsed with an initiator buffer containing a polymeric polycation (such as Polybrene) that is adsorbed to the wall surface followed by introduction of the running buffer containing a polymeric polyanion (such as dextran sulfate) that is forming a second layer and thus providing the negative charge for a strong electroosmotic flow towards the cathode [35].

An important prerequisite of an accurate determination of analytes is a technique that allows the interference-free, selective determination of the compounds of interest. Thus, baseline resolution of the analytes has to be achieved in CZE employing UV detection in order to avoid the necessity of sophisticated data evaluation procedures or mass spectrometry. After selection of the dynamic coating with the best overall performance characteristics, the aim of the further investigations was therefore to improve the method, with special emphasis on the separation between disialo-Tf and trisialo-Tf (i.e. the resolution between CDT and non-CDT $\mathrm{Tf}$ isoforms) and to assess the precision performance of the assay. The interpretation of clinical laboratory data and the conclusions drawn from these data are based on the comparison of a test result with a reference interval. Thus, the elucidation of a reliable reference interval is an important 
task that has to be done before a test can be introduced into the routine arena. Therefore, the goal of the third project of this thesis was to establish the reference interval for CDT analyzed by CZE and to investigate the impact of instrumental parameters and data integration schemes on the reference limits for CDT. The last part of this dissertation was to evaluate the final, optimized assay under routine conditions via analysis of a large number of sera from patients of the hepatology outpatient clinic. Special emphasis was directed towards the identification of atypical Tf patterns obtained with rare genetic Tf variants and the investigation of abnormal non-Tf peaks in the $\gamma$-and $\beta$-regions of the electropherograms. 


\section{B. Results and Discussion}

The content of this dissertation is subject of four publications. Thus, the following chapters contain these papers that describe the evolution of the project, starting with the evaluation of different dynamic capillary coatings and ending with the application of the final method in the routine arena. 


\section{B.1. EVALUATION AND OPTIMIZATION OF CAPILLARY ZONE ELECTROPHORESIS WITH DIFFERENT DYNAMIC CAPILLARY COATINGS FOR THE DETERMINATION OF CARBOHYDRATE- DEFICIENT TRANSFERRIN IN HUMAN SERUM}

Published in: Journal of Chromatography A, 979 (2002) 43 - 57 


\title{
EVALUATION AND OPTIMIZATION OF CAPILLARY ZONE ELECTROPHORESIS WITH DIFFERENT DYNAMIC CAPILLARY COATINGS FOR THE DETERMINATION OF CARBOHYDRATE-DEFICIENT TRANSFERRIN IN HUMAN SERUM
}

\author{
Christian Lanz ${ }^{1}$, Martina Kuhn ${ }^{1}$, Federica Bortolotti ${ }^{2}$, Franco Tagliaro ${ }^{2}$ and Wolfgang \\ Thormann ${ }^{1}$ \\ ${ }^{1}$ University of Bern, Department of Clinical Pharmacology, \\ 3010 Bern, Switzerland. \\ ${ }^{2}$ University of Verona, Department of Public Medicine and Health - Unit of Forensic \\ Medicine, Verona, Italy.
}




\section{Abstract}

Serum transferrin (Tf) comprises several isoforms with up to two complex oligosaccharide chains containing 0 to 8 sialic acid residues and neutral sugars. The major glycoform, known as tetrasialo-Tf, contains 4 sialic acid residues and accounts for about $80 \%$ of whole $\mathrm{Tf}$ in human serum. Carbohydrate-deficient transferrin (CDT) encompasses isoforms that are deficient in carbohydrate chains and consequently in sialic acid residues (including asialo-, monosialo- and disialo-Tf) and is a well known marker for chronic alcohol abuse. Recently capillary zone electrophoresis (CZE) has been reported as a tool extremely effective for the simultaneous, individual, quantitative determination of CDT isoforms. Three CZE methods that feature different dynamic capillary coatings were evaluated and optimized for CDT determination in human serum of alcohol abusers and control subjects. CZE separation was performed in alkaline borate buffers after serum sample saturation with iron, electropherograms were detected at $200 \mathrm{~nm}$, data were evaluated as area \% of disialo-Tf in relation to tetrasialo-Tf and peak identification was accomplished via relative migration times to tetrasialo-Tf, immunosubtraction and enzymatic sequential cleavage of sialic acid residues. Dynamic capillary coatings with diaminobutane, spermine and a double coating produced by commercially available proprietary agents were investigated and found to be suitable for determination of CDT in human serum. For all three approaches, best results were obtained in $50 \mu \mathrm{m}$ I.D. fused-silica capillaries of $50 \mathrm{~cm}$ effective length and a capillary cartridge temperature of $20-25^{\circ} \mathrm{C}$. Using $3 \mathrm{mM}$ 1,4-diaminobutane (DAB) or $0.02 \mathrm{mM}$ spermine in a $\mathrm{pH} 8.3$ borate-based running buffer provided data of remarkable similarity with resolution of di-, tri-, tetra- and pentasialo-Tf within 15 to $18 \mathrm{~min}$. With the double coating, asialo-Tf and $\mathrm{Tf}$ isoforms with 2 to 6 sialic acid residues were baseline separated. Compared to the two amine-based procedures, the run times were found to be somewhat shorter, the detector signals higher, the applied power level significantly lower and the reproducibility better. 


\section{Introduction}

Alcohol misuse and alcohol dependence are common in many different cultures and there are no doubts that among the substances active on the central nervous system, ethanol has the heaviest medical, social and economic impact. Alcohol abuse can generally be divided in "high risk drinking" that means a regular daily intake of about $60 \mathrm{~g}$ or more of ethanol in males (about $\geq 40 \mathrm{~g}$ in females), and "alcohol dependence" which encompasses excessive drinking with consequential physical and psychological harm. Therefore, early diagnosis of alcohol abuse has become a very important topic not only in clinical medicine, but also in psychiatry, occupational, traffic and forensic medicine. In these fields, different questionnaires and biological markers like alanine aminotransferase (ALT), aspartate aminotransferases (AST), erythrocyte mean corpuscular volume (MCV), $\gamma$-glutamyltransferase (GGT), serum urate and triglycerides are currently used in assisting the effort of an objective and reliable diagnosis of alcoholism [1,2]. In the 1970s, Stibler et al. proposed carbohydrate-deficient transferrin (CDT) as a new biological marker for chronic or repeated excessive alcohol intake [35]. Transferrin (Tf), the most important iron transporting protein, is a glycoprotein with a molecular mass of about 79'570 daltons that consists of a single peptide chain of 679 amino acids. The $\mathrm{N}$-terminal (amino acids 1-336) and C-terminal (amino acids 337-679) domains are globular and encompass an iron binding site each. Tf has two N-linked carbohydrate chains at the positions 413 and 611 that are composed of a bitetraantennary carbohydrate chain containing $\mathrm{N}$-acetylglucosamine, mannose, galactose and terminal sialic acid residues [6,7]. Based on a different content of end standing sialic acid residues (zero up to eight), human serum comprises different isoforms of $\mathrm{Tf}$ $[1,7]$. At alkaline $\mathrm{pH}$, sialic acid residues are negatively charged and the $\mathrm{Tf}$ isoforms thus differ in their isoelectric points and their electrophoretic behavior. The major Tf isoform in humans consists of four sialic acid residues (tetrasialo-Tf) with an isoelectric point of 5.4 (after complete iron saturation). Following chronic alcohol ingestion of 50 $80 \mathrm{~g}$ ethanol per day over a period of one to two weeks, the amounts of some less glycosilated forms with isoelectric points of 5.7 (disialo-Tf) and 5.9 (asialo-Tf) are reported to increase $[1,2]$. The majority of these isoforms were found to comprise one and no carbohydrate chain, respectively [8,9]. Isoforms with $\mathrm{pI}$ values $\geq 5.7$, corresponding to molecules with two or less sialic acid residues, are collectively named CDT. CDT is considered to be the most specific marker available today for the detection of chronic, excessive alcohol intake [1]. 
CDT was first monitored by isoelectric focusing combined with immunofixation, zone immunoelectrophoresis or Western blotting. Later on, assays based upon ionexchange and other forms of high-performance liquid chromatography were developed $[1,2,10,11]$. Sensitivity and specificity of these procedures were reported to be good. Productivity, however, is too low for routine use. Thus, various two-step assay kits based on the removal of non-CDT isoforms by ion exchange chromatography on disposable microcolumns followed by immunochemical determination of the remaining Tf isoforms were developed. The commercially available kits differ mainly in the efficiency of the chromatographic separation step and in the immunochemical detection method (radioimmunoassay, enzyme immunoassay, immunoturbidimetric assay, etc), whereas the antisera used are in any case directed towards whole human $\mathrm{Tf}$ (no specificity for CDT). Because of insufficient resolution between isoforms with similar pI's in the microcolumn chromatographic step, the composition of the collected CDT fraction not only encompasses CDT according to the above stated definition, but may also contain an unspecified amount of trisialo Tf. Another problem is the well-known susceptibility of specific (abnormal genetic Tf variants) and nonspecific interferences of the immunometric determination. CDT immunoassays are therefore prone to analytical inaccuracy $[1,2,12-14]$.

During the last decade, a great deal of interest was focused on the determination of Tf isoforms and particularly CDT by capillary electrophoresis (CE) [15-29]. Capillary isoelectric focusing (CIEF) was found to be capable of resolving the major forms of human [15,16] and bovine [19] Tf. Due to the complexity and insufficient reproducibility of these approaches, however, CIEF could not be adopted for the determination of CDT in human serum. The use of free solution capillary zone electrophoresis (CZE) was more successful. Kilàr and Hjertén reported the resolution of the Tf isoforms with zero and up to six sialic acid residues in polyacrylamide-coated capillaries and with a $\mathrm{pH} 8.4$ borate/Tris buffer containing 0.3 mM EDTA [17]. Oda and Landers published a detailed study on the CZE separation of Tf isoforms from purified Tf of different species, including humans. Resolution of the major Tf isoforms was studied in bare fused-silica capillaries with borate and phosphate buffers containing different cationic buffer additives for column conditioning, including 1,4-diaminobutane (DAB) also referred to as putrescine or tetramethylenediamine, decamethonium and hexamethonium [18]. Employing bare fused-silica capillaries and a Tris/glutamic acid buffer at $\mathrm{pH}$ around 8.6, a CZE study of abnormal glycoforms of serum $\mathrm{Tf}$ in carbohydrate-deficient glycoprotein syndrome type I was published by Iourin et al. [20]. Prasad et al. [21] and Oda et al. [22] studied the CZE determination of CDT in DB-17 
coated capillaries using borate buffers with different alkylated celluloses as additives. Significant improvement was achieved with respect to analysis time, but the procedure required immunopurification of serum before CZE. Furthermore, Trout et al. [27] and Beisler et al. [28] used fluorocarbon-coated capillaries achieving good resolution of the Tf isoforms. However, longevity of the covalently-coated capillary was too short and the separation performance was affected by an aging process of the capillary. Independently, Tagliaro et al. published a detailed investigation of the CZE separation of human $\mathrm{Tf}$ isoforms in bare fused-silica capillaries with I.D.'s of $20-100 \mu \mathrm{m}$ combined with different buffers. Good separation within reasonable time and without complicated sample preparation was obtained with a short capillary $(30 \mathrm{~cm}$ to the detector) of $20 \mu \mathrm{m}$ I.D. [23]. Elongation of the capillary length and addition of $1.5 \mathrm{mM}$ or $3 \mathrm{mM}$ of DAB to a $100 \mathrm{mM}$ borate buffer lead to further improvements [24,25] such that the assay could be introduced in Verona (Italy) for forensic confirmation of sera that were found to be positive using CDT immunoassays. The efficacy of DAB in preventing proteins to adhere to the capillary wall was also demonstrated by Giordano et al. who investigated the effect of increasing DAB concentrations $(0-4 \mathrm{mM})$ added to a borate buffer in a $50 \mu \mathrm{m}$ I.D. bare fused-silica capillary [26]. In agreement with the work from the laboratory in Verona, good separation of the Tf isoforms was achieved in presence of $3 \mathrm{mM}$ DAB. Finally, Wuyts et al. [29] investigated the analysis of CDT with proprietary reagents that provide a dynamic double coating of the capillary produced by adsorption of a polycation prior to application of a second layer composed of a polyanion. This coating procedure is comparable to that described by Katayama et al. [30]. Unfortunately, as disialo- and trisialo Tf were not separated with the buffer used, this assay cannot be employed for the determination of CDT [31].

The main problem in CZE of proteins is the interaction of the highly charged proteins with the silanol groups of the capillary wall, resulting in a strong impairment of separation performance. Different approaches to overcome protein adsorption have been proposed, including the use of electrolyte solutions at extreme $\mathrm{pH}$ values, buffers of high ionic strength, buffer additives that dynamically coat the capillary wall and covalently coated capillaries [32,33]. Based upon the recent literature [15-29], the use of buffer additives that provide dynamic capillary coatings appears to be the most promising approach for analysis of CDT in human serum. The aim of this work was (i) to revisit and thereby optimize the use of DAB [24-26] as capillary conditioner, (ii) to test the employment of spermine (N,N'-bis(3-aminopropyl)1,4-butanediamine) as alternative buffer additive, an oligoamine that is known to be an effective quencher of protein adsorption [32,34] and that has been successfully employed for CZE analysis of 
ovalbumin glycoforms [35], and (iii) to evaluate a newly developed commercial kit that provides a dynamic double coating (CEofix CDT-kit of Analis, Namur, Belgium). The performance of the three dynamic coatings were studied in terms of resolution of $\mathrm{Tf}$ isoforms, run times, signal magnitudes and reproducibility. For optimization, capillary dimensions, buffer composition, amount of sample injected, applied power and capillary cartridge temperature were varied. The ultimate goal of this study was to find the most suitable approach for the routine arena.

\section{Experimental}

\subsection{Chemicals}

If not stated otherwise, chemicals were of analytical grade. Disodium tetraborate decahydrate and sodium hydrogen carbonate were from Merck (Darmstadt, Germany). Caffeine was obtained from Inselspital-Apotheke (Bern, Switzerland). Ferric(III)chloride hexahydrate and spermine tetrahydrochloride ( $\geq 99.5 \%$ ) were purchased from Fluka (Buchs, Switzerland). DAB (approx. $98 \%$ ), neuraminidase type $\mathrm{X}$ from clostridium perfringens (210 units/mg solid, 240 units/mg protein) and iron saturated holo-Tf (minimum $98 \%$ ) were from Sigma (St. Louis, MO, USA). Rabbit anti-human Tf antibody (titer: 2’800 mg/l) was obtained from Dako (Glostrup, Denmark). Buffers and reagents of the Analis CEofix-CDT-kit for quantitation of CDT with the Beckman Coulter P/ACE MDQ (Kit No. 10-004740) were kindly provided by Analis (Namur, Belgium). It is important to note that this kit comprises a similar but not identical buffer than that used by Wuyts et al. [29].

\subsection{Serum samples}

Patient sera were obtained from the departmental analytical laboratory where they were received for determination of CDT. Our own sera were used as sera of healthy persons. All blood samples were drawn by venipuncture and collected in native plastic tubes without additives (Monovette, white cap, Sarstedt, Sevelen, Switzerland). After clotting the blood samples were centrifuged for $10 \mathrm{~min}$ at $3500 \mathrm{rpm}$ at room temperature. The supernatant was transferred into $8.0 \mathrm{ml}$ polypropylene tubes. All sera were stored at $-20^{\circ} \mathrm{C}$ until use. 


\subsection{Sample preparation}

Sera were slowly defrosted and vortex mixed at room temperature. For the experiments with DAB and spermine, $200 \mu \mathrm{l}$ of serum was combined with $5 \mu 1$ of an aqueous $10 \mathrm{mM}$ ferric(III)chloride solution and $5 \mu \mathrm{l}$ of a $500 \mathrm{mM}$ solution of sodium hydrogen carbonate in a plastic vial. After vortex mixing for $30 \mathrm{~s}$ the sample was incubated at room temperature for $30 \mathrm{~min}$. Then, 5 or $10 \mu \mathrm{l}$ of the sample was transferred into a sample vial and, if not stated otherwise, diluted ten-fold with water. The solution was gently mixed by aspiration and release of part of the fluid with a plastic pipette. For the determination of the electroosmotic flow (EOF), an aqueous caffeine $(100 \mu \mathrm{g} / \mathrm{ml})$ solution was used to dilute the iron saturated serum. For the experiments with the Analis CDT-kit, $60 \mu \mathrm{l}$ of serum and $60 \mu \mathrm{l}$ of the ferric solution of the kit were mixed directly in the microtiter plate (Beckman Coulter, Fullerton, USA) according to the manufacturer's instructions. The sample was mixed gently by aspiration and release of part of the fluid with a pipette. No incubation time was required to saturate the serum with iron.

\subsection{Buffer preparation}

For the experiments with $\mathrm{DAB}$, borate stock solutions with concentrations of $12.5-200 \mathrm{mM}$ tetraborate and a $\mathrm{pH}$ of 8.3 (adjusted via addition of $6 \mathrm{M} \mathrm{HCl}$ ) and a 90 $\mathrm{mM}$ stock solution of DAB containing $0.05 \mathrm{mM} \mathrm{HCl}$ were prepared and kept at $4{ }^{\circ} \mathrm{C}$. The running buffers with the desired concentrations of tetraborate and DAB were prepared daily. Similarly, for the experiments with spermine, $\mathrm{pH} 8.3$ borate stock solutions (25,50 and $100 \mathrm{mM}$ tetraborate) and two stock solutions comprising 50 and 1 $\mathrm{mM}$ spermine, respectively, were prepared and stored at $4^{\circ} \mathrm{C}$. The running buffers were prepared daily. The higher concentrated stock solution of spermine was used for preparing buffers with spermine concentrations from $3.0-0.1 \mathrm{mM}$, whereas the $1 \mathrm{mM}$ stock solution was employed to obtain spermine concentrations between 0.05 and 0.01 $\mathrm{mM}$. For the experiments with the double coating, the buffers were employed as supplied in the kit. 


\subsection{Instrumentation and running conditions for the experiments with DAB and spermine}

CZE measurements were performed on a P/ACE MDQ capillary electrophoresis system (Beckman Coulter) which was equipped with fused-silica capillaries (Polymicro Technologies, Phoenix, AZ, USA) of 15, 25, 50 and $75 \mu \mathrm{m}$ I.D. and 60.2, 50.2 and 40.2 $\mathrm{cm}$ (50.0, 40.0 and $30.0 \mathrm{~cm}$ to the detector) total length. All capillaries had an O.D. of about $375 \mu \mathrm{m}$. If not stated otherwise, the temperature controls of the capillary cartridge and the sample tray were set to $25^{\circ} \mathrm{C}$ and $20^{\circ} \mathrm{C}$, respectively, samples were introduced by positive pressure and the applied voltage was $20 \mathrm{kV}$. Reversed polarity (cathode at the injection end) was used for the experiments with spermine concentrations $\geq 0.5 \mathrm{mM}$. UV detection was effected by using a $200 \mathrm{~nm}$ interference filter. Data registration, evaluation and storage were carried out with the 32 Karat Software, version 4.01 (Beckman).

New capillaries were sequentially rinsed with $1 \mathrm{M} \mathrm{NaOH}, 0.2 \mathrm{M} \mathrm{NaOH}$, water and running buffer (90 min each for capillaries of 15 and $25 \mu \mathrm{m}$ I.D., 20 - 30 min each for capillaries 50 and $75 \mu \mathrm{m}$ I.D.) by applying a pressure of $60 \mathrm{psi}$ at the inlet end. Then the capillaries were filled with running buffer and kept at room temperature for $60 \mathrm{~min}$ followed by application of $20 \mathrm{kV}$ for $30 \mathrm{~min}$. At the beginning of a day, capillaries were typically rinsed for 5-6 min with $1 \mathrm{M} \mathrm{NaOH}$ with a pressure of $60 \mathrm{psi}$ from the inlet side, followed by rinsing for $10 \mathrm{~min}$ with $0.2 \mathrm{M} \mathrm{NaOH}$ and for 12-15 min with running buffer with a pressure of 60 psi from the outlet side. The conditioning step at the beginning of the day was completed by application of $20 \mathrm{kV}$ for $10-15 \mathrm{~min}$. Between runs, capillaries were rinsed from the outlet side with $0.2 \mathrm{M} \mathrm{NaOH}$ at 60 psi for 4 min (15 and $25 \mu \mathrm{m}$ I.D.) or $2 \mathrm{~min}$ (50 and $75 \mu \mathrm{m}$ I.D.) and with running buffer at 30 to 40 psi for $6 \min (15$ and $25 \mu \mathrm{m}$ I.D.) or $3 \min (50$ and $75 \mu \mathrm{m}$ I.D.). At the end of the day, the capillaries were first washed with $1 \mathrm{M} \mathrm{NaOH}$ from the outlet side followed by rinses from the inlet side with $1 \mathrm{M} \mathrm{NaOH}, 0.2 \mathrm{M} \mathrm{NaOH}$ and water $(60 \mathrm{psi}, 10 \mathrm{~min}$ each with 15 and $25 \mu \mathrm{m}$ I.D., 5 min each with 50 and $75 \mu \mathrm{m}$ I.D.). Capillaries and their ends were kept in water during storage. 


\subsection{Instrumentation and running conditions for the experiments with the double coating}

If not stated otherwise, CZE measurements were performed according to the instructions of the manufacturer of the CEofix CDT-kit (Analis) on the P/ACE MDQ instrument (Beckman Coulter). The system was equipped with a fused-silica capillary (Analis) of $50 \mu \mathrm{m}$ I.D. and $60.2 \mathrm{~cm}(50.0 \mathrm{~cm}$ length to the detector) total length. The O.D. was $360 \mu \mathrm{m}$. The temperature controls of the cartridge and sample tray were set to $40{ }^{\circ} \mathrm{C}$ and $15{ }^{\circ} \mathrm{C}$, respectively. New capillaries were rinsed for $10 \mathrm{~min}$ with $0.2 \mathrm{M}$ $\mathrm{NaOH}$ by applying a pressure of $40 \mathrm{psi}$ at the inlet end. The same washing step was employed at the beginning of each day. At the end of a day the capillary was rinsed for $5 \mathrm{~min}$ with $0.2 \mathrm{M} \mathrm{NaOH}$ and for $5 \mathrm{~min}$ with water by applying a pressure of 60 psi at the inlet end. Capillaries were stored wet with the ends kept in water. Between runs capillaries were rinsed with $0.2 \mathrm{M} \mathrm{NaOH}$ for 1 min with a pressure of 60 psi from the inlet end and then for $1.5 \mathrm{~min}$ with $0.2 \mathrm{M} \mathrm{NaOH}$ by applying simultaneously a constant current of $80 \mu \mathrm{A}$ and a pressure of 20 psi at the inlet end. At the beginning of a new run, the capillary was rinsed from the inlet to the outlet side for $1.5 \mathrm{~min}$ with the initiator buffer and for 2 min with the run buffer of the CDT kit by applying a pressure of 15 psi. Sample was introduced by applying a vacuum of $0.3 \mathrm{psi}$ for $10 \mathrm{~s}$ at the outlet side. A voltage of $28 \mathrm{kV}$ was applied (anode at the injection end) and the current was about 26 $\mu$ A. Detection and data processing was performed as in Section 2.5. Selected experiments were performed with lower cartridge temperature and/or increased capillary length.

\subsection{Evaluation of electropherograms}

Data evaluation was based upon peak area measurements. For comparison of all approaches described in this manuscript, the amount of CDT was evaluated as area \% of disialo-Tf in relation to tetrasialo-Tf and referred to as Tf-index [25]. Resolution (Rs) was calculated with the 32 Karat Software (Beckman Coulter) using Rs $=1.18\left(\mathrm{t}_{2}\right.$ $\left.\mathrm{t}_{1}\right) /\left(\left(\mathrm{W}_{\mathrm{h} / 2}\right)_{1}+\left(\mathrm{W}_{\mathrm{h} / 2}\right)_{2}\right)$ where $\mathrm{t}_{\mathrm{i}}$ and $\left(\mathrm{W}_{\mathrm{h} / 2}\right)_{\mathrm{i}}$ represent the detection time and peak width at half height of firstly $(i=1)$ and secondly $(i=2)$ detected peak, respectively. An Rs value of $\geq 1.4$ represents baseline resolution. Peak asymmetry (tailing factor, defined as half of the peak width at $5 \%$ of peak height divided by the distance from the peak maximum to 
the leading edge of the peak at the position of 5\% peak height) and efficiency expressed in $\mathrm{plates} / \mathrm{m}$ were also determined with this software.

\subsection{Immunosubtraction and enzymatic digestion}

For immunosubtraction of $\mathrm{Tf}, 80 \mu \mathrm{l}$ of serum was vortex mixed in a plastic vial with $160 \mu \mathrm{l}$ of anti-human Tf immunoglobulins and incubated at room temperature for $1.5 \mathrm{~h}$. After centrifugation at $10^{\prime} 000 \mathrm{~g}$ for $25 \mathrm{~min}$ at $4^{\circ} \mathrm{C}$, the supernatant was collected immediately and measured without further preparation. For data comparison with untreated sample, serum was equally diluted with water after iron saturation and analyzed under the same conditions. For digestion with neuraminidase, the enzyme was reconstituted to $1 \mathrm{U} / \mathrm{ml}$ with $50 \mathrm{mM}$ sodium acetate ( $\mathrm{pH}$ 5.0, adjusted with acetic acid). $160 \mu 1$ of serum was saturated in a plastic vial with $160 \mu 1$ of the ferric solution of the CEofix CDT-kit and combined with $160 \mu 1$ of the enzyme solution. After vortex mixing, the sample was first incubated at room temperature for $5 \mathrm{~h}$ and then at $37{ }^{\circ} \mathrm{C}$ until about $72 \mathrm{~h}$. Aliquots were analyzed with the double coating method.

\subsection{CDT determination with the \%CDT TIA test}

CDT was determined with the turbidimetric immunoassay \%CDT TIA of AxisShield (distributed through Bio-Rad, Hercules, CA, USA) according to the recommendations of the kit manufacturer using the Cobas Mira Plus (Roche Diagnostics, Rotkreuz, Switzerland). A CDT value $<2.6 \%$ of total Tf was considered to be within the normal range.

\section{Results and Discussion}

\subsection{Experiments with DAB}

The basis for the experiments with DAB was the work of Tagliaro et al. [24,25] in which CDT was analyzed in $20 \mu \mathrm{m}$ I.D. capillaries employing a borate $\mathrm{pH} 8.3$ buffer containing 1.5 to $3 \mathrm{mM}$ DAB. The performance of DAB was further investigated in capillaries of different inner diameters $(15,25,50$ and $75 \mu \mathrm{m}$, Fig. 1) and by varying borate concentration, applied voltage, operational temperature and amount of sample 
injected. Peak identification of the minor isoforms was accomplished by calculating the relative migration times to tetrasialo-Tf. Iron saturated sera were diluted ten-fold with water or an aqueous caffeine solution $(100 \mu \mathrm{g} / \mathrm{ml})$. Selected electropherograms obtained for the serum of a healthy subject and using capillaries of $50 \mathrm{~cm}$ effective length are presented in Fig. 1.

Using a $25 \mu \mathrm{m}$ I.D. capillary, $2.25 \mathrm{mM}$ DAB in a $100 \mathrm{mM}$ borate buffer, a voltage of $20 \mathrm{kV}$ (current about $30 \mu \mathrm{A}$ ) and a cartridge temperature of $25{ }^{\circ} \mathrm{C}$, nice resolution of the major $\mathrm{Tf}$ isoforms, including di- and trisialo-Tf, was obtained within $18 \mathrm{~min}$, whereas pentasialo-Tf was only partially separated from tetrasialo-Tf (second graph from top in Figs. 1A and 1B, Table 1). Asialo-Tf, present in sera from alcohol abusers, was not detected (data not shown), and hexasialo-Tf could not be identified. Depending on the injection time which was varied between $13.5 \mathrm{~s}$ and $30 \mathrm{~s}(0.5 \mathrm{psi}$ pressure, plug $\leq 0.76 \%$ of capillary volume), the height of the tetrasialo-Tf peak was $2.5-7 \mathrm{mAU}$ and the signal to noise ratio for disialo-Tf (smallest peak) was $\leq 6$. The effect of cartridge temperature on the separation of $\mathrm{Tf}$ isoforms was investigated between $20{ }^{\circ} \mathrm{C}$ and $45{ }^{\circ} \mathrm{C}$ (steps of $5{ }^{\circ} \mathrm{C}$ ). The temperature has an influence on the viscosity and conductivity of the buffer system and thus the mobilities of the analytes and electroosmosis. With increased temperature, the power dissipation in the capillary (expressed in $\mathrm{W} / \mathrm{m}$, for selected values refer to Table 1) and the intracapillary temperature become higher and therefore may require lowering the voltage or decreasing the electrolyte concentration of the buffer. Detection time could be reduced from about $21 \mathrm{~min}$ at $20^{\circ} \mathrm{C}$ to about $11 \mathrm{~min}$ at $45{ }^{\circ} \mathrm{C}$ (electropherograms not shown). For the entire temperature interval, the applied power and current increased from 0.93 to $1.48 \mathrm{~W} / \mathrm{m}$ and 28 to $44 \mu \mathrm{A}$, respectively. The separation characteristics did not alter much except that the resolution between di- and trisialo-Tf was found to deteriorate as function of temperature. A similar effect on the migration times was observed by increasing the voltage from $20 \mathrm{kV}$ to $30 \mathrm{kV}$ (steps of $5 \mathrm{kV}$, temperature of $25^{\circ} \mathrm{C}$ ). The detection time could thereby be reduced from about $18 \mathrm{~min}$ to about $11.5 \mathrm{~min}$. The current increased from 30 to $48 \mu \mathrm{A}$ and the power applied changed from 1.02 to 2.38 $\mathrm{W} / \mathrm{m}$, indicating that the intracapillary temperature is increasing as well [36]. Despite of the relatively high power level associated with the application of $30 \mathrm{kV}$, the separation was found to be almost as good as that obtained with $20 \mathrm{kV}$ and $25^{\circ} \mathrm{C}$ and was clearly better than with $20 \mathrm{kV}$ and $45{ }^{\circ} \mathrm{C}$ (data not shown). No further improvement of peak resolution was obtained by varying the borate concentration up to $200 \mathrm{mM}$ and by dilution of the iron saturated sera five-fold or twenty-fold. Increased peak resolution 
between disialo- and trisialo-Tf was noted after reducing the I.D. of the capillary to 15 $\mu \mathrm{m}$ (top graphs in panels A and B of Fig. 1, Table 1). However, much smaller signals were observed and the signal-to-noise ratio for disialo- $\mathrm{Tf}$ became very low. Thus, no further work with $15 \mu \mathrm{m}$ I.D. capillaries was undertaken.

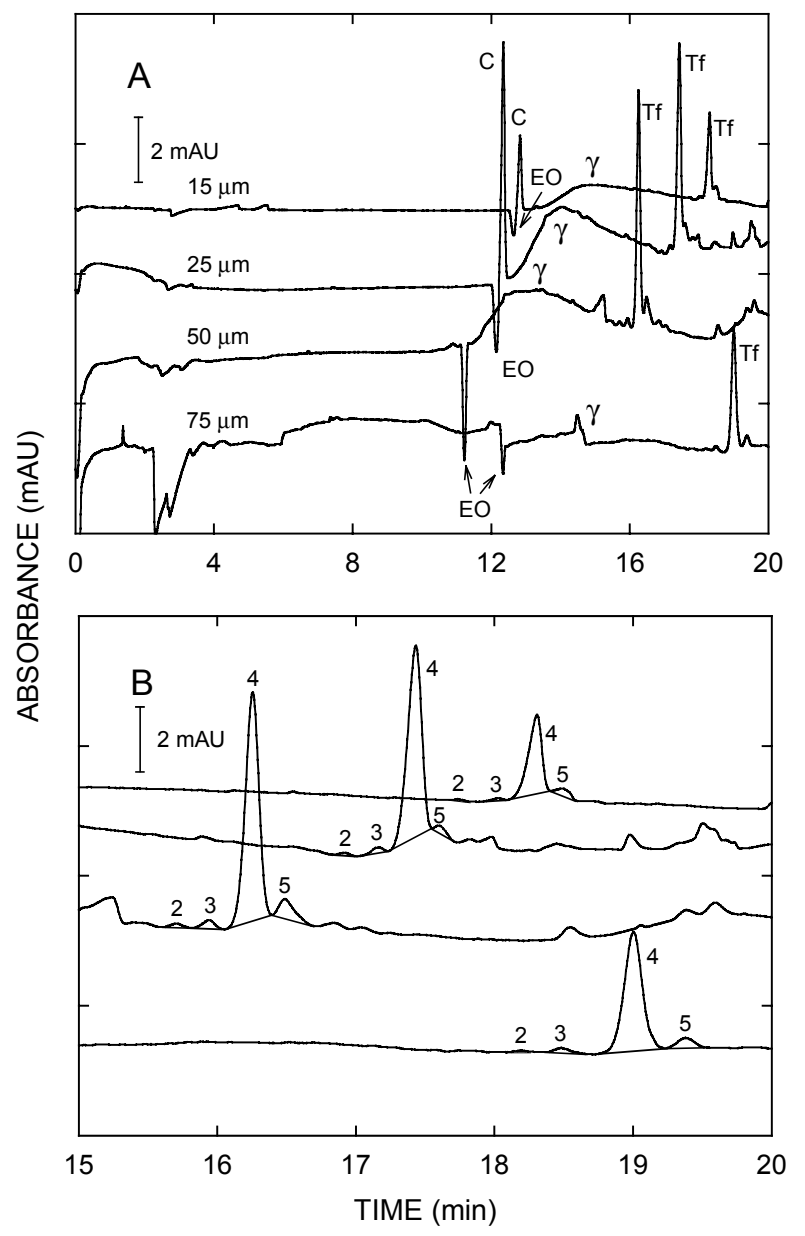

Figure 1: Optimized CZE electropherograms of a serum from a healthy individual using $\mathrm{pH} 8.3$ borate buffers with DAB in capillaries of 15, 25, 50 and $75 \mu \mathrm{m}$ I.D. (graphs from top to bottom) and $50 \mathrm{~cm}$ effective length. The entire electropherograms and the sections with the $\mathrm{Tf}$ pattern on an elongated $\mathrm{x}$-axis scale are presented in panels $\mathrm{A}$ and $\mathrm{B}$, respectively. The DAB concentrations were 2.25, 2.25, 3.0 and 3.0 $\mathrm{mM}$, respectively, and the borate concentrations were 100, 100, 50 and $25 \mathrm{mM}$, respectively. The injection times (applied pressure) were $70 \mathrm{~s}(0.5 \mathrm{psi}), 19 \mathrm{~s}(0.5 \mathrm{psi}), 4 \mathrm{~s}(0.5 \mathrm{psi})$ and $5 \mathrm{~s}(0.2 \mathrm{psi})$, respectively. The applied voltages were $20 \mathrm{kV}($ I.D. $<75 \mu \mathrm{m})$ and $18 \mathrm{kV}(\mathrm{I} . \mathrm{D}=75 \mu \mathrm{m})$ and the currents were 10.0, 30.6, 57.4 and $78.0 \mu \mathrm{A}$, respectively. The temperature of the cartridge was $25{ }^{\circ} \mathrm{C}$ in all cases. Other conditions are listed in Table 1. For the sake of clarity, electropherograms are presented with yscale offsets. Key: 2: disialo-Tf, 3: trisialo-Tf, 4: tetrasialo-Tf, 5: pentasialo-Tf, C: caffeine, $\gamma:$ gamma region, EO: electroosmosis. 

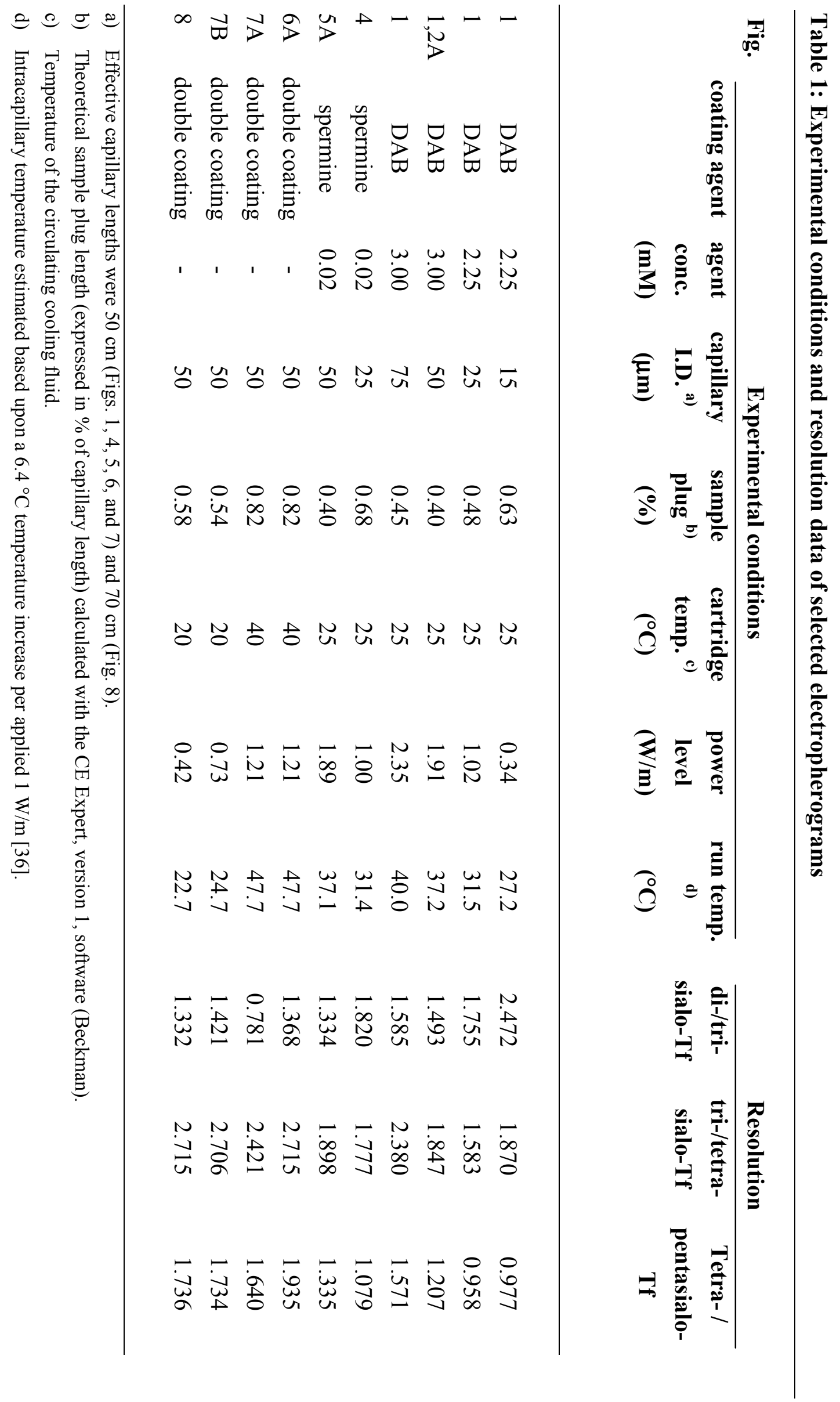
Enlargement of the capillary I.D. lead to electropherograms with increased signal magnitudes (Fig. 1) and increased operational temperatures (Table 1). Best results were obtained with a $50 \mu \mathrm{m}$ I.D capillary, an effective capillary length of $50 \mathrm{~cm}$, a $50 \mathrm{mM} \mathrm{pH}$ 8.3 borate buffer containing $3.0 \mathrm{mM} \mathrm{DAB}$, an applied voltage of $20 \mathrm{kV}$ and an operational temperature of $25{ }^{\circ} \mathrm{C}$ (second graph from bottom in Fig. 1, Fig. 2). For a pressure of $0.5 \mathrm{psi}$, the injection time was optimized to $4 \mathrm{~s}$, corresponding to a theoretical plug length of $0.40 \%$ of the capillary length. The electroosmotic mobility $\left(\mathrm{m}_{\mathrm{eo}}\right)$ and applied power levels were calculated to be $2.24 \times 10^{-4} \mathrm{~cm}^{2} / \mathrm{Vs}$ and $1.91 \mathrm{~W} / \mathrm{m}$, respectively, and the estimated intracapillary temperature was estimated to be $37.2{ }^{\circ} \mathrm{C}$. Under these conditions, all $\mathrm{Tf}$ isoforms were detected within $17 \mathrm{~min}$. Baseline separation of di- and trisialo-Tf was achieved and the resolution between tetrasialo-Tf and pentasialo-Tf was good (Table 1). The height of the tetrasialo-Tf peak was about $7 \mathrm{mAU}$ and the signal to noise ratio for disialo-Tf in the pattern of healthy individuals (Figs. 1 and 2A) was between 10 and 15. Except for peak resolution, no further improvement was obtained by enlarging the I.D. of the capillary to $75 \mu \mathrm{m}$ (bottom graphs in Fig. 1, Table 1).

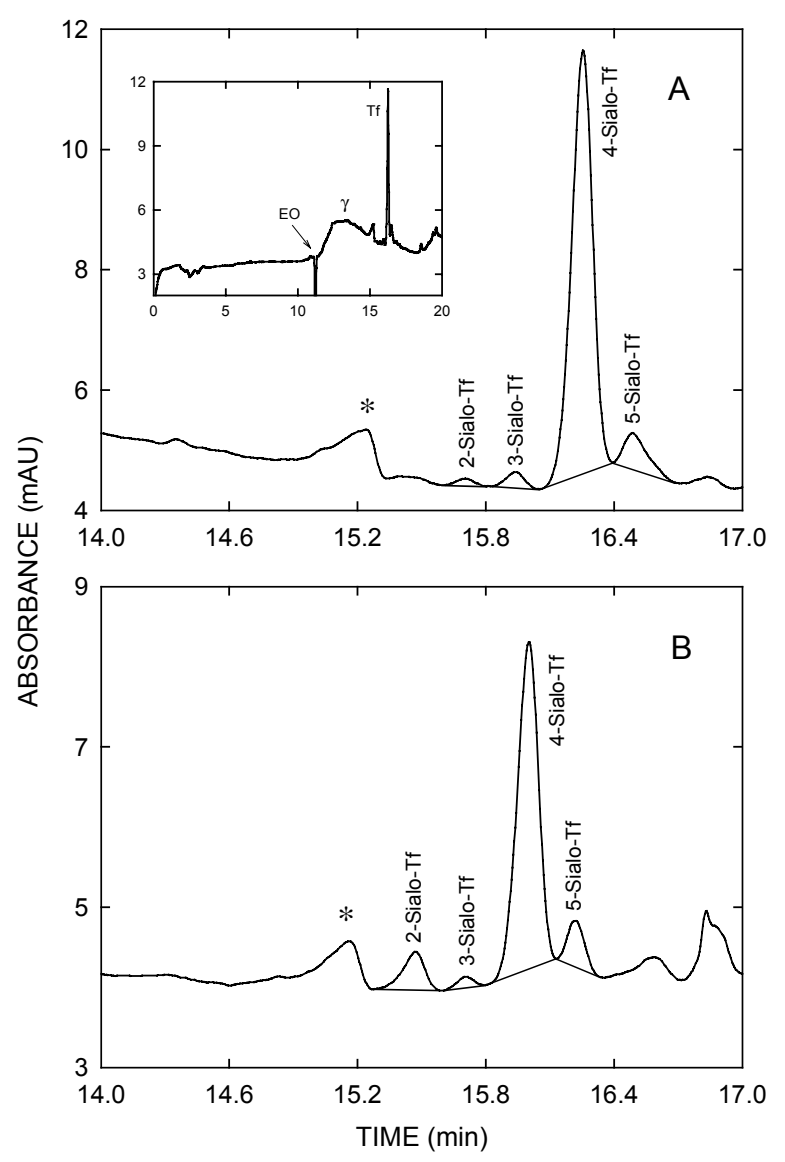

Figure 2: Electropherograms in presence of $3.0 \mathrm{mM}$ DAB obtained with (A) a serum of a healthy individual and (B) a serum of an alcohol abuser in a $50 \mu \mathrm{m}$ I.D. capillary of $50 \mathrm{~cm}$ effective length. Other experimental conditions as for the $50 \mu \mathrm{m}$ I.D. electropherogram of Fig. 1. The insert in panel A depicts the entire electropherogram. Key as for Fig. 1 
The data presented in Fig. 2 represent typical serum Tf patterns of a healthy individual (Fig. 2A) and an alcohol abuser (Fig. 2B). Data were evaluated as area \% of disialo-Tf in relation to tetrasialo-Tf and referred to as Tf-index, the normal value assessed in $20 \mu \mathrm{m}$ I.D. capillaries being $\leq 2.27 \%$ [25]. The two Tf-indices were thereby calculated to be $1.35 \%$ and $12.06 \%$, respectively. Thus, CZE with DAB as capillary conditioner was found to correctly classify the two sera. The same was found to be true for the \%CDT TIA test that revealed values of 2.0 and $6.3 \%$, respectively. It is important to note, that asialo-Tf could not be detected with the CZE assay (Fig. 2B). Furthermore, hexasialo-Tf could not be identified. The peaks marked with asterisks are of unknown origin and were present in most electropherograms (for exceptions refer to Fig. 1). Typical reproducibility data for peak efficiency, peak shape and resolution obtained via analysis of a serum of a healthy individual are summarized in Table 2, whereas corresponding RSD values for detection time, peak area and Tf-index are presented in Table 3.

\subsection{Experiments with spermine}

The effect of varying spermine concentrations on electroosmosis and the separation of $\mathrm{Tf}$ isoforms was investigated in capillaries of $25 \mu \mathrm{m}$ I.D., $50 \mathrm{~cm}$ effective length and a cartridge temperature of $25^{\circ} \mathrm{C}$. First, a solution of purified human holo-Tf $(0.5 \mathrm{mg} / \mathrm{ml}$ in water $)$ and iron saturated serum of a healthy person that was diluted with an aqueous solution of caffeine $(100 \mu \mathrm{g} / \mathrm{ml})$ were analyzed employing $100 \mathrm{mM}$ borate buffers of a pH 8.3 comprising spermine concentrations between 3.0 to $0.02 \mathrm{mM}$. Selected electropherograms are presented in Figs. 3 and 4. For monitoring of electroosmosis in absence of proteins, caffeine $(1 \mathrm{mg} / \mathrm{ml}$ in water) was injected. With spermine concentrations between 3.0 and $0.5 \mathrm{mM}$ and application of $20 \mathrm{kV}$, caffeine could not be detected within $90 \mathrm{~min}$ of current flow. This means that under these conditions the EOF was markedly reduced, abolished or even reversed. The data suggested that $m_{\mathrm{eo}}<0.28 \times 10^{-4} \mathrm{~cm}^{2} /$ Vs. Similarly, from experiments with reversed polarity, it could be concluded that $\mathrm{m}_{\mathrm{eo}}>-0.40 \times 10^{-4} \mathrm{~cm}^{2} / \mathrm{Vs}$. For detection of $\mathrm{Tf}$ the polarity had to be reversed $(-20 \mathrm{kV}$, first and second graphs from top of Fig. 3, top graph of Fig. 4). Under these conditions highly charged anions are reaching the detector first. For analysis of serum in presence of $3.0 \mathrm{mM}$ spermine, a large splitted peak representing albumin was detected at $18 \mathrm{~min}$, followed by several small peaks probably encompassing $\alpha 1$ - and $\alpha 2$ globulins and finally Tf at about 27 min (top graph of Fig. 4). No separation between disialo- and trisialo-Tf was obtained. Shortening of the capillary 
or application of a higher voltage did not lead to improved Tf isoform separation (data not shown). The same was found to be true with a $0.5 \mathrm{mM}$ spermine concentration (second graph from top in Fig. 3).

Having a spermine concentration of $0.1 \mathrm{mM}$, normal polarity (detector at the cathode) and otherwise identical experimental conditions as described above, it was possible to detect the EOF within about $30 \mathrm{~min}\left(\mathrm{~m}_{\mathrm{eo}}=0.84 \times 10^{-4} \mathrm{~cm}^{2} / \mathrm{Vs}\right)$, but $\mathrm{Tf} \mathrm{did}$ not reach the detector within $70 \mathrm{~min}$ (second graph from bottom in Fig. 3). Thus, for analysis of $\mathrm{Tf}$, electroosmosis appears to be to too weak. With 0.05 to $0.02 \mathrm{mM}$ spermine, electroosmosis was observed to increase as the spermine concentration was decreased, and almost fully resolved Tf patterns were noted. Best results were obtained with $0.02 \mathrm{mM}$ spermine $\left(\mathrm{m}_{\mathrm{eo}}=1.71 \times 10^{-4} \mathrm{~cm}^{2} / \mathrm{Vs}\right)$ and having an effective capillary length of $50 \mathrm{~cm}$ (bottom graphs in Figs. 3 and 4). With an injection time of $27 \mathrm{~s}$ (pressure: $0.5 \mathrm{psi}$ ), the observed separation characteristics were found to be similar to those obtained with $2.25 \mathrm{mM}$ DAB in a fused-silica capillary of the same length and I.D.

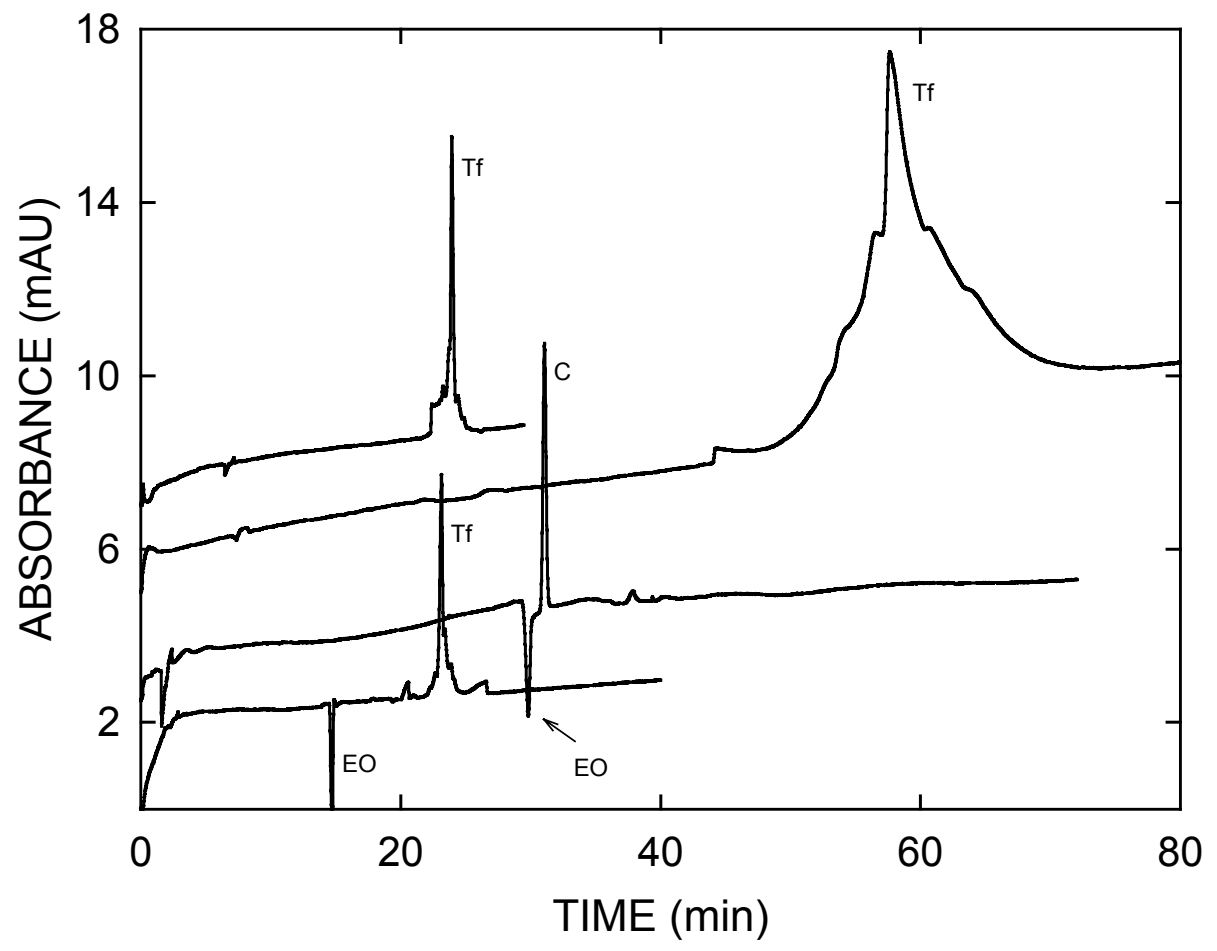

Figure 3: Electropherograms obtained with Tf standard $(0.5 \mathrm{mg} / \mathrm{ml}$ in water $)$ using $\mathrm{pH} 8.3,100 \mathrm{mM}$ borate buffers with spermine in a capillary of $25 \mu \mathrm{m}$ I.D. and $50 \mathrm{~cm}$ effective length. The spermine concentration varied between 3.0, 0.5, 0.1 and $0.02 \mathrm{mM}$ (graphs from top to bottom, respectively). The applied voltages were $-20 \mathrm{kV},-20 \mathrm{kV}, 20 \mathrm{kV}$ and $20 \mathrm{kV}$, respectively, and the currents were about $30 \mu \mathrm{A}$ in all four cases. Injection was effected for $27 \mathrm{~s}$ at $0.5 \mathrm{psi}$ and the cartridge temperature was $25{ }^{\circ} \mathrm{C}$ in all cases. For the sake of clarity, electropherograms are presented with y-scale offsets. Key as for Fig. 1. 


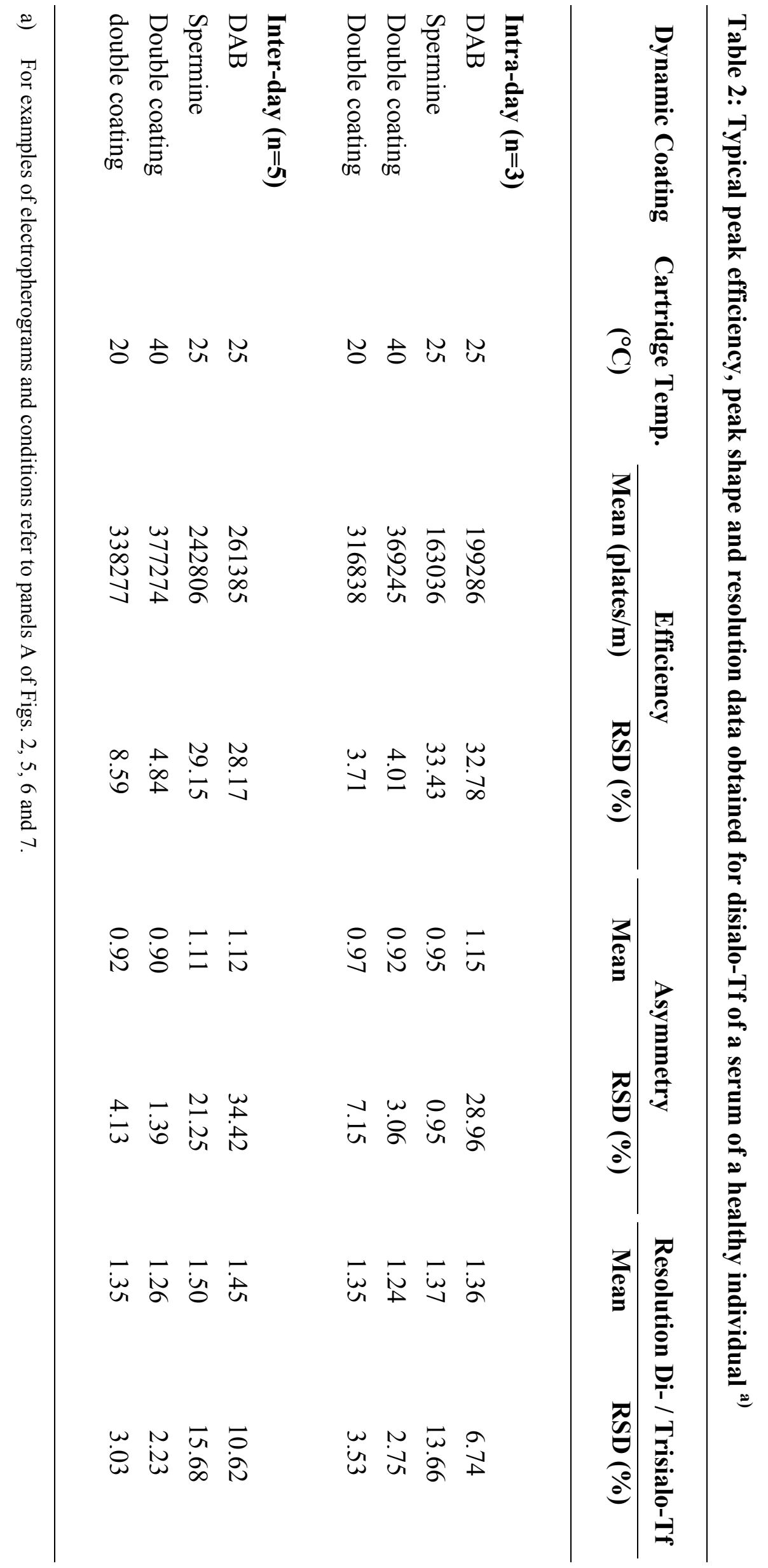




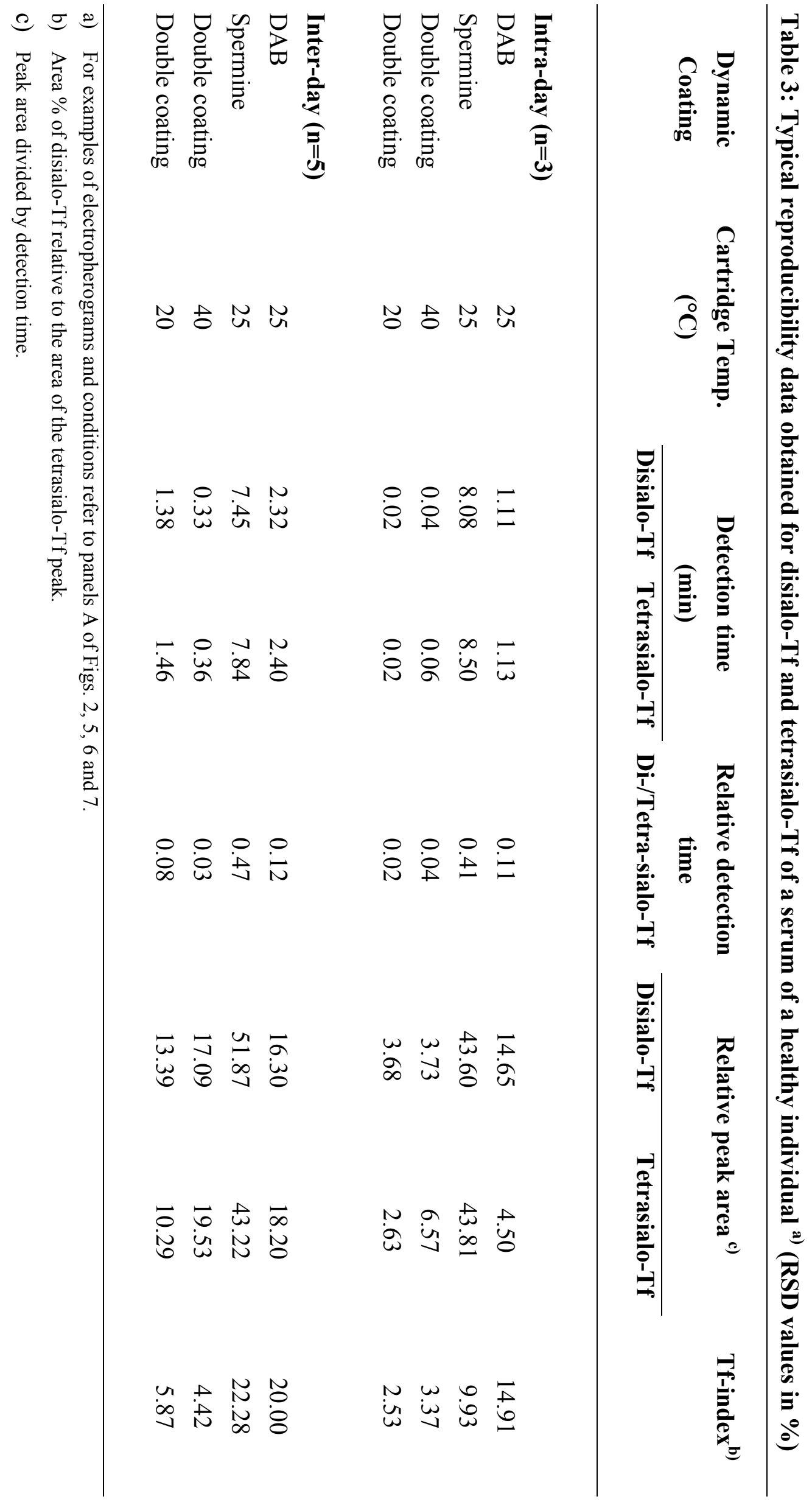


(Table 1). The analysis time, however, was somewhat longer with spermine. Baseline separation of di- and trisialo-Tf was obtained within $27 \mathrm{~min}$ and pentasialo-Tf was not fully separated from tetrasialo-Tf (Table 1). No improvements were noted with shorter capillaries (tested with $30 \mathrm{~cm}$ effective length) and with $15 \mu \mathrm{m}$ I.D. capillaries. However, increased detector signals and an overall better separation was obtained with $50 \mu \mathrm{m}$ I.D. capillaries of $50 \mathrm{~cm}$ total length (Fig. 5, Table 1). As was the case with DAB (Fig. 2), the borate concentration had to be reduced to $50 \mathrm{mM}$. With a voltage of $20 \mathrm{kV}$, the temperature control of the cartridge being set to $25{ }^{\circ} \mathrm{C}$, an injection time of $4 \mathrm{~s}$ (pressure: $0.5 \mathrm{psi}$ ) and a spermine concentration of $0.020 \mathrm{mM}$, Tf with its highly resolved isoforms reached the detector within about 16.0 to $17.5 \mathrm{~min}$ (Fig. 5). The concentration of the capillary conditioner was found to be critical. With $0.025 \mathrm{mM}$ spermine, all Tf isoforms were detected within 20-26 min, whereas with $0.01 \mathrm{mM}$

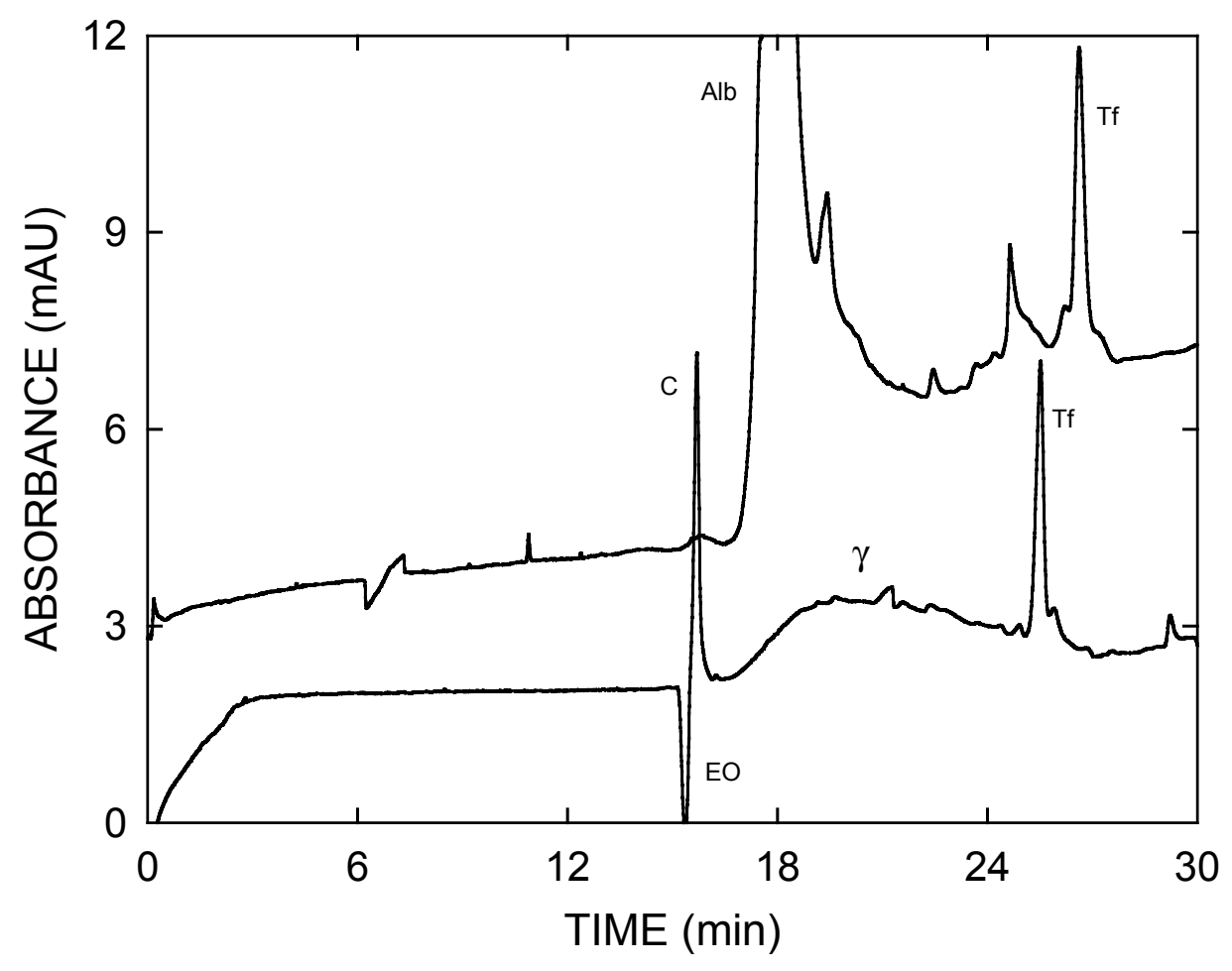

Figure 4: Electropherograms obtained with a serum of a healthy individual (10-fold diluted after iron saturation) in presence of $3.0 \mathrm{mM}$ spermine (upper graph, reversed polarity) and of $0.02 \mathrm{mM}$ spermine (lower graph, normal polarity). Other conditions as for top and bottom graphs, respectively, of Fig. 3. Alb refers to albumin. 
spermine, incompletely resolved $\mathrm{Tf}$ isomers reached the detector within about $12 \mathrm{~min}$. Furthermore, within a set of experiments, a tendency to shorter analysis times was noted using this capillary conditioning agent.

The data presented in Fig. 5 represent typical serum Tf patterns of a healthy individual (Fig. 5A) and an alcohol abuser (Fig. 5B), samples that were also analyzed with DAB (Fig. 2). The Tf-indices were calculated to be $1.34 \%$ and $9.04 \%$, respectively, indicating that the separation performance for di- to pentasialo-Tf was the same as with $3 \mathrm{mM}$ DAB (Fig.2). It is important to note, that asialo-Tf and hexasialo-Tf could not be detected as well (Fig. 5B). The peaks marked with an asterisk are of unknown origin and were present in most electropherograms. Typical reproducibility data for peak efficiency, peak shape and resolution obtained via analysis of a serum of a

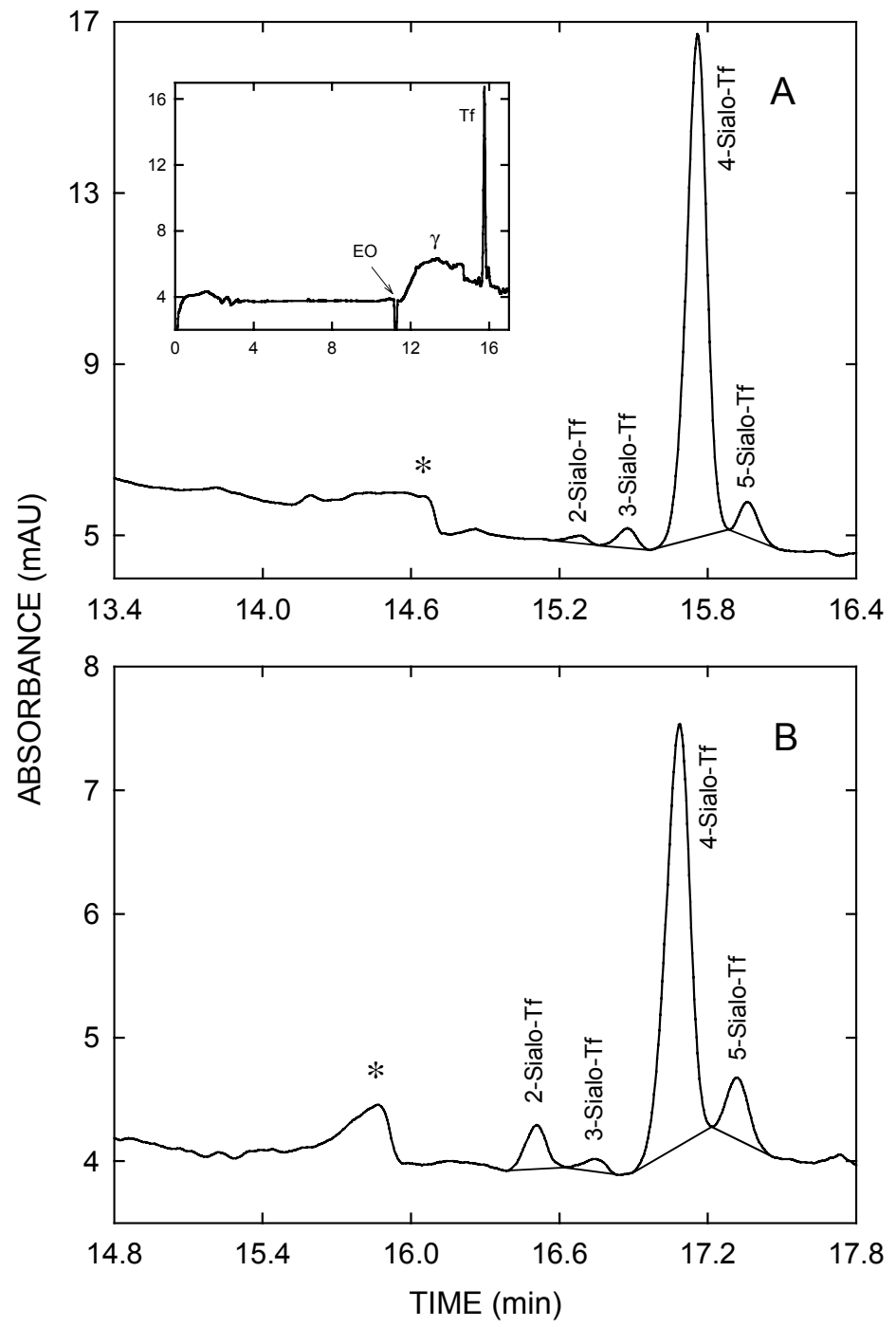

Figure 5: Electropherograms in presence of $0.02 \mathrm{mM}$ spermine obtained with (A) a serum of a healthy individual and (B) a serum of an alcohol abuser in a $50 \mu \mathrm{m}$ I.D. capillary of $50 \mathrm{~cm}$ effective length. The borate buffer concentration was $50 \mathrm{mM}$, the injection time (pressure) was $4 \mathrm{~s}$ (0.5 psi), the voltage was $20 \mathrm{kV}$ and the currents were about $57 \mu \mathrm{A}$. The temperature of the cartridge was set to $25{ }^{\circ} \mathrm{C}$. The insert in panel A depicts the entire electropherogram. Key as for Fig. 1. 
healthy individual are summarized in Table 2, whereas corresponding RSD values for peak detection time, peak area and the Tf-index are presented in Table 3. Compared to the configuration with $\mathrm{DAB}$, imprecision of detection times, relative detection time and peak areas were found to be larger. The RSD of the Tf-index, however, was comparable. Furthermore, it was interesting to find that the electroosmotic mobility and power level for the data of Fig. $5 \mathrm{~A}$ were almost identical to those of the data with $3 \mathrm{mM}$ DAB presented in Fig. 2A (2.24 x $10^{-4} \mathrm{~cm}^{2} / \mathrm{Vs}$ and $1.9 \mathrm{~W} / \mathrm{m}$, respectively).

\subsection{Experiments with dynamic double coating}

Experiments with the proprietary double coating were primarily performed according to the instructions of the manufacturer of the CEofix CDT-Kit (see section 2.6). For analysis of a serum of a healthy individual, resolution of $\mathrm{Tf}$ isoforms with 2 to 6 sialic acid residues was obtained within 7 min (Fig. 6A, Table 1). Additionally, asialoTf could be nicely detected in selected sera from alcohol abusers (Fig. 6B). The electroosmotic mobility was calculated to be $3.73 \times 10^{-4} \mathrm{~cm}^{2} / \mathrm{Vs}$, a value that is $66.5 \%$ higher compared to those characteristic for the corresponding assays with DAB (Fig. 2) and spermine (Fig. 5). The power level was significantly lower, namely $1.21 \mathrm{~W} / \mathrm{m}$ compared to $1.9 \mathrm{~W} / \mathrm{m}$. The peak height of the main peak (tetrasialo-Tf) was about 40 mAU, i.e. the signal was about $4-6$ times higher than the signal of the corresponding peak when analyzed with the assays comprising DAB or spermine as capillary conditioning agents (compare with Figs. 2 and 5).

The data presented in Fig. 6 represent typical serum Tf patterns of a healthy individual (Fig. 6A) and an alcohol abuser (Fig. 6B). The two Tf-indices were calculated to be $1.30 \%$ and $20.06 \%$, respectively. Thus, CZE with the double coating was found to correctly classify the two sera. The same was found to be true for the $\%$ CDT TIA test that revealed values of 2.0 and $13.6 \%$, respectively. It is important to note the serum of the alcohol abuser whose data are shown in Fig. 6B is not the same as was used to produce the patterns shown in Figs. $2 \mathrm{~B}$ and $5 \mathrm{~B}$. The latter serum lead to a Tf-index of $7.7 \%$ and a very small asiolo-Tf peak and was thus not depicted. Typical reproducibility data for peak efficiency, peak shape and resolution obtained via analysis of a serum of a healthy individual are summarized in Table 2, whereas corresponding RSD values for peak detection time, peak area and the Tf-index are presented in Table 3. Compared to the assays with DAB and spermine, RSD values for all parameters were found to be significantly lower. Thus, the double coating appears to abolish the protein 
wall interactions more efficiently and/or provides improved resurfacing for each analysis.

Although not visible for the data presented in Fig. 6A and with the calculated resolution value given in Table 1, resolution between di- and trisialo-Tf was typically noted to be lower compared to the cases with DAB and spermine (Table 2). For selected patient sera with high amounts of trisialo-Tf (Fig. 7B), this was found to become a problem. For analysis under normal conditions, incomplete separation of di- and trisialo-Tf was noted (Fig. 7A, Rs $=0.78$, Tf-index $=0.35 \%$ ). The amount of trisialo-Tf relative to tetrasialo-Tf was determined to be $12.5 \%$, a value that is about 2.5 -fold higher compared to the $4.9 \%$ determined for the serum of Fig. 6A. Analysis of the same serum with the cartridge temperature reduced to $20{ }^{\circ} \mathrm{C}$ (electroosmotic mobility of 2.56

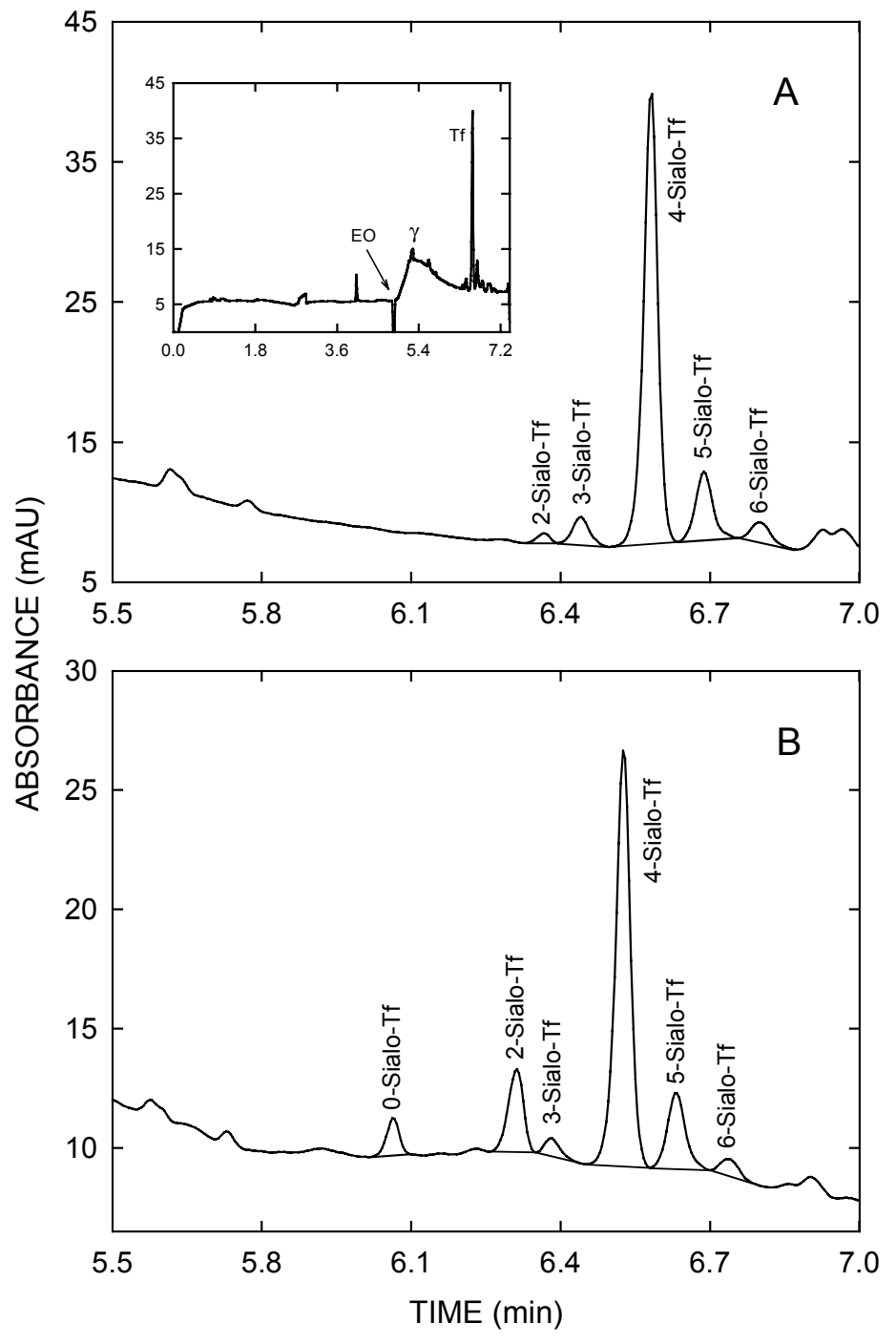

Figure 6: Electropherograms obtained with the dynamic double coating for analysis of (A) a serum of a healthy individual and (B) a serum of an alcohol abuser having a $50 \mu \mathrm{m}$ I.D. capillary of $50 \mathrm{~cm}$ effective length. The injection time was $10 \mathrm{~s}$ (0.3 psi, vacuum), the voltage was $28 \mathrm{kV}$ and the currents were $26.0 \mu \mathrm{A}$. The temperature of the cartridge was $40{ }^{\circ} \mathrm{C}$. The insert in panel $\mathrm{A}$ depicts the entire electropherogram. Key as for Fig. 1. 
$\mathrm{x} 10^{-4} \mathrm{~cm}^{2} / \mathrm{Vs}$, power level of $0.73 \mathrm{~W} / \mathrm{m}$ ) and otherwise identical conditions, revealed a run time of about $10.5 \mathrm{~min}$, a resolution between di- and trisialo-Tf of 1.42, a Tf-index of $0.75 \%$ and a relative amount of trisialo-Tf of $12.6 \%$ (Fig. 7B). Furthermore, this patient serum was also analyzed in an elongated capillary $(70 \mathrm{~cm}$ instead of $50 \mathrm{~cm}$ effective length) held at $20^{\circ} \mathrm{C}$ which resulted in a run time of about 20 min and a Tfindex of $0.82 \%$ (Fig. 8, upper graph). Using immunosubtraction as described in section 2.9, all assigned peaks were determined to represent Tf (Fig. 8, lower graph). For comparison of the electropherograms it is important to note that the immunosubtracted sample was monitored with a time shift of about $10 \mathrm{~s}$, an effect that originates from the difference of the sample matrix. Furthermore, misinterpretation of the electropherograms was prevented with incubation of serum with neuraminidase for increasing times and analysis of a range of samples with which the sequential cleavage

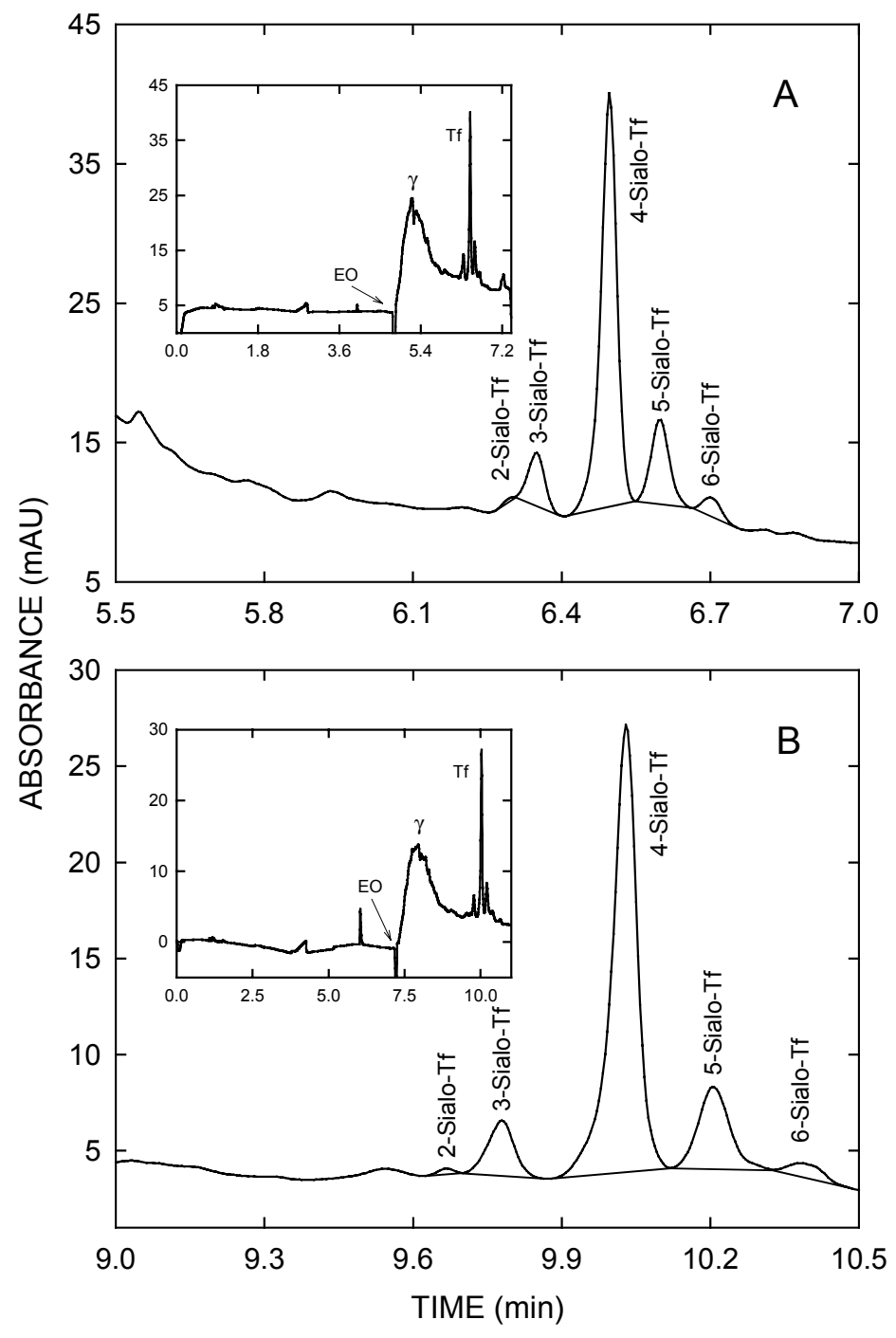

Figure 7: Electropherograms obtained with a patient serum containing a high amount of trisialo-Tf after analysis with the dynamic double coating at (A) 40 ${ }^{\circ} \mathrm{C}$ and (B) $20{ }^{\circ} \mathrm{C}$ and otherwise identical conditions as for Fig. 6 . The current for panel B was 15.7 $\mu \mathrm{A}$. The inserts depict the entire electropherograms. Key as for Fig. 1. 
of the sialic acid moieties could be visualized (data not shown). Elongation of the capillary provided no significant improvement of separation (Table 1). Thus, based upon the resolution increase (Table 2) and the excellent reproducibility (Table 3) obtained for operation at $20{ }^{\circ} \mathrm{C}$, it can be concluded that the use of the $50 \mathrm{~cm}$ capillary should be sufficient for monitoring CDT in clinical and forensic practice.

According to the CZE data presented in Figs. 7 and 8, the analyzed serum with increased trisialo-Tf was classified as having a normal Tf-index $(<2.27 \%$ [25]). However, the \%CDT TIA test revealed a CDT value of $3.6 \%$ which is considered to be positive according to the reference values proposed in the instruction manual of the test kit $(<2.6 \%)$ and used in clinical practice $(<3.0 \%$ [12]). The inaccuracy of the \%CDT turbidimetric immunoassay appears to be obvious for such cases. Thus, should a positive immunoassay test result lead to serious consequences, it should always be confirmed by a more specific method, such as CZE [25] or HPLC [12,14].

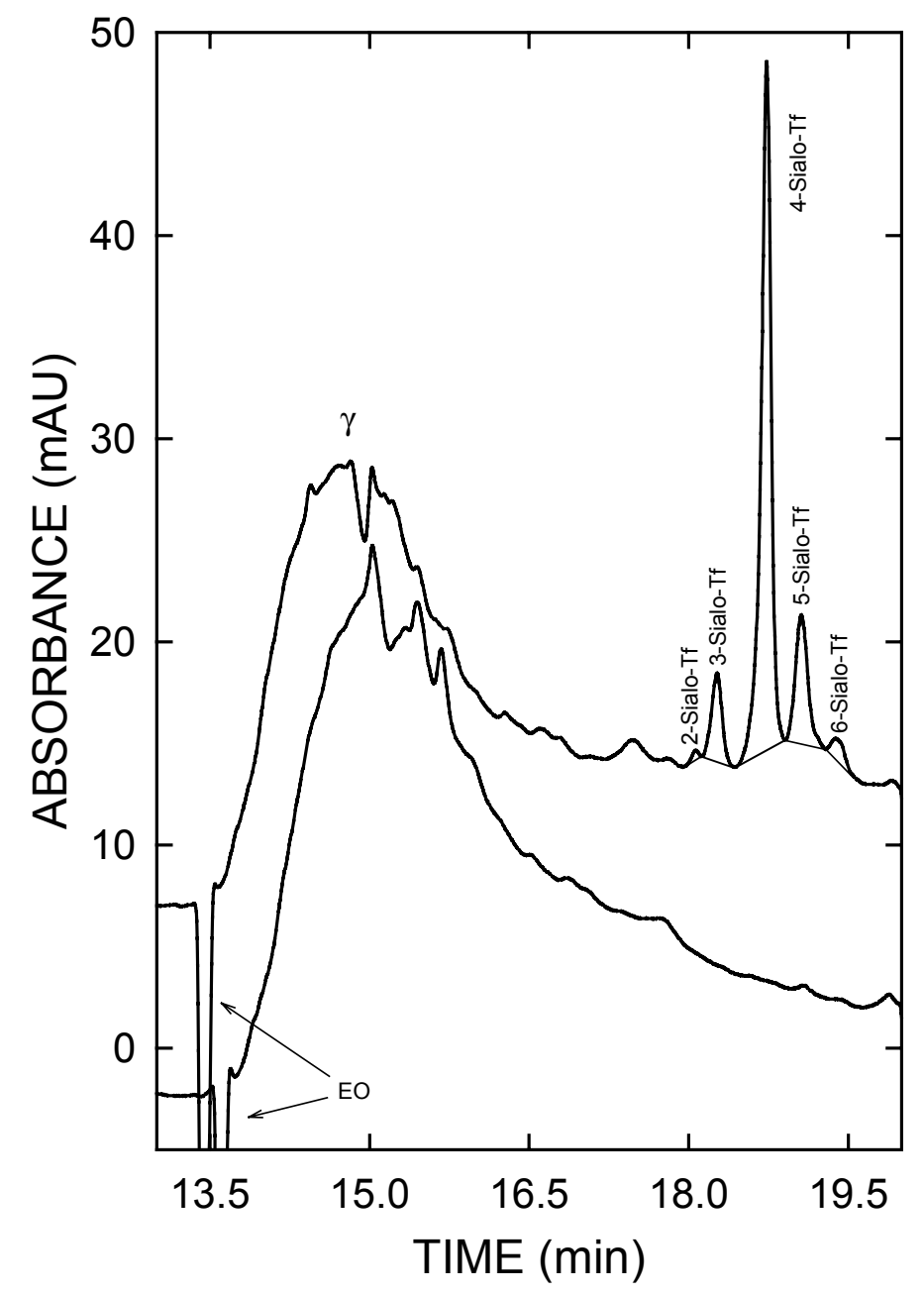

Figure 8: Electropherograms obtained with a patient serum containing a high amount of trisialo-Tf before (upper graph) and after (lower graph) immunosubtraction for analysis with the dynamic double coating in a $50 \mu \mathrm{m}$ I.D. capillary of $70 \mathrm{~cm}$ effective length and a cartridge temperature of $20{ }^{\circ} \mathrm{C}$. Injection was performed by applying a vacuum of $0.6 \mathrm{psi}$ for $10 \mathrm{~s}$. The current was $12.1 \mu \mathrm{A}$. Other conditions as for Fig. 6. For the sake of presentation, the upper graph is depicted with a $9 \mathrm{mAU}$ yaxis shift. 


\section{Conclusions}

The major problems in analyzing CDT with CZE are the interaction of the macromolecules with the capillary wall and the maintenance of the capillary surface to guarantee reproducibility. As permanently coated capillaries are expensive and were found to deteriorate with time, the use of plain capillaries together with buffer additives that provide a dynamic coating appears to be the most effective approach in terms of both analytical performance and cost. In our work with the P/ACE MDQ instrument, configurations with two amine modifiers, DAB and spermine, and a proprietary commercially available double coating were investigated and found to be suitable for determination of CDT in human serum. For all three approaches, best electropherograms in terms of $\mathrm{Tf}$ isoform resolution, detection signals and run times were obtained in $50 \mu \mathrm{m}$ I.D. capillaries of $50 \mathrm{~cm}$ effective length, application of normal polarity (electroosmosis towards the cathode) and a capillary cartridge temperature of $20-25^{\circ} \mathrm{C}$. Having $3 \mathrm{mM} \mathrm{DAB}$ or $0.02 \mathrm{mM}$ spermine in a $\mathrm{pH} 8.3$ borate based running buffer provided data of remarkable similarity. With these approaches and having an applied power of $1.9 \mathrm{~W} / \mathrm{m}$ at $20 \mathrm{kV}$, di-, tri-, tetra- and pentasialo- $\mathrm{Tf}$ can be separated and detected within 15 to $18 \mathrm{~min}$. Asialo-Tf and hexasialo-Tf, however, cannot be monitored. Data evaluated based upon area \% of disialo-Tf in relation to tetrasialo-Tf were found to be comparable and reproducible. Furthermore, the use of DAB was noted to provide better reproducibility of detection times and higher temperatures lead to reduced run times but insufficient separability in both cases. With the double coating and the buffers provided in the commercial CDT-kit, asialo-Tf and Tf isoforms with 2 to 6 sialic acid residues are monitored. The capillary is first rinsed with an initiator (containing a proprietary polycation in a malic acid/arginine buffer at $\mathrm{pH} 4.8$ ) followed by introduction of the running buffer (containing a proprietary polyanion in a Tris/boric acid buffer at $\mathrm{pH}$ 8.5) and analysis of the iron saturated sample. Using the recommended cartridge temperature of $40{ }^{\circ} \mathrm{C}$, excellent reproducibility and short run times of about 7 min were noted. Compared to the assays based upon DAB and spermine, signal magnitudes were determined to be significantly higher and the applied power level lower $(1.21 \mathrm{~W} / \mathrm{m}$ at $28 \mathrm{kV})$. The resolution of di- and trisialo-Tf, however, was incomplete with sera containing high amounts of trisialo-Tf. To obtain complete $\mathrm{Tf}$ isoform resolution for these cases, the cartridge temperature had to be lowered to $20{ }^{\circ} \mathrm{C}$ which provided run times of about $10 \mathrm{~min}$ (power level of $0.73 \mathrm{~W} / \mathrm{m}$ ) and excellent reproducibility. Based upon the evaluations reported in this paper, the use of the double coating at $20{ }^{\circ} \mathrm{C}$ together with the commercially available CEofix-CDT-kit currently appears to be the best approach for CZE analysis of CDT in a routine arena. 


\section{Acknowledgements}

The authors acknowledge the receipt of the Analis CEofix CDT kits from its manufacturer (Analis, Namur, Belgium). This work was supported by the Liver Foundation, Bern, Switzerland, the Swiss National Science Foundation and MIUR (Italian Ministry of Education, University and Research, project \# 2001065132). 


\section{References}

[1] T. Arndt, Clin. Chem. 47 (2001) 13-27.

[2] F. Tagliaro, F. Bortolotti, F. Crivellente, F. Cittadini, Forensic Sci. Rev. 12 (2000)

134-148.

[3] H. Stibler, K.G. Kjellin, J. Neurol. Sci. 30 (1976) 269-285.

[4] H. Stibler, C. Allgulander, S. Borg, K.G. Kjellin, Acta med. Scand. 204 (1978) 49-56

[5] H. Stibler, S. Borg, C. Allgulander, Acta med. Scand. 206 (1979) 275-281.

[6] P. Sillanaukee, N. Strid, J.P. Allen, R.Z. Litten, Alcohol. Clin. Exp. Res. 25 (2001) 34-40.

[7] G. de Jong, H.G. van Ejik, Electrophoresis 9 (1988) 589-598.

[8] E. Landberg, P. Påhlsson, A. Lundblad, A. Arnetorp, J.-O. Jeppsson, Biochem. Biophys. Res. Commun. 210 (1995) 267-274.

[9] T. Inoue, M. Yamauchi, K. Ohkawa, Electrophoresis 20 (1999) 452-457.

[10] J.-O. Jeppsson, H. Kristensson, C. Fimiani, Clin. Chem. 39 (1993) 2115-2120.

[11] E. Werle, G.E. Seitz, B. Kohl, W. Fiehn, H.K. Seitz, Alcohol Alcohol. 32 (1997) 71-77.

[12] A. Helander, M. Fors, B. Zakrisson, Alcohol Alcohol. 36 (2001) 406-412.

[13] M. Lipowski, L. Dibbelt, M. Seyfarth, Clin. Biochemistry, 33 (2000) 635-641.

[14] U. Turpeinen, T. Methuen, H. Alfthan, K. Laitinen, M. Salaspuro, U.-H. Stenman, Clin. Chem. 47 (2001) 1782-1787.

[15] S. Molteni, W. Thormann, J. Chromatogr. 638 (1993) 187-193.

[16] F. Kilàr, S. Hjertén, Electrophoresis 10 (1989) 23-29.

[17] F. Kilàr, S. Hjertén, J. Chromatogr. 480 (1989) 351-357.

[18] R.P. Oda, J.P. Landers, Electrophoresis 17 (1996) 431-437.

[19] L. Yang, Q. Tang, A.K. Harrata, C.S. Lee, Anal. Biochem. 243 (1996) 140-149.

[20] O. Iourin, T.S. Mattu, N. Mian, G. Keir, B. Winchester, R.A. Dwek, P.M. Rudd, Glycoconj. J. 13 (1996) 1031-1042.

[21] R. Prasad, R.L. Stout, D. Coffin, J. Smith, Electrophoresis 18 (1997) 1814-1818.

[22] R.P. Oda, R. Prasad, R.L. Stout, D. Coffin, W.P. Patton, D.L. Kraft, J.F. O’Brien, J.P. Landers, Electrophoresis 18 (1997) 1819-1826.

[23] F. Tagliaro, F. Crivellente, G. Manetto, I. Puppi, Z. Deyl, M. Marigo, Electrophoresis 19 (1998) 3033-3039. 
[24] F. Crivellente, G. Fracasso, R. Valentini, G. Manetto, A.P. Riviera, F. Tagliaro, J. Chromatogr. B 739 (2000) 81-93.

[25] F. Tagliaro, F. Bortolotti, M. Zuliani, F. Crivellente, G. Manetto, V.L. Pascali, M. Marigo, J. Cap. Elec. 6 (1999) 137-143.

[26] B.C. Giordano, M. Muza, A. Trout, J.P. Landers, J. Chromatogr. B 742 (2000) 79-89.

[27] A.L. Trout, R. Prasad, D. Coffin, A. DiMartini, T. Lane, C. Blessum, N. Khatter, J.P. Landers, Electrophoresis 21 (2000) 2376-2383.

[28] A.T. Beisler, R..H. Kelly, J.P. Landers, Anal. Biochem. 285 (2000) 143-150.

[29] B. Wuyts, J.R. Delanghe, I. Kasvosve, A. Wauters, H. Neels, J. Janssens, Clin. Chem. 47 (2001) 247-255.

[30] H. Katayama, Y. Ishihama, N. Asakawa, Anal. Chem. 70 (1998) 2254-2260.

[31] F. Tagliaro, F. Bortolotti, R.M. Dorizzi, M. Marigo, Clin. Chem. 48 (2002) 208209.

[32] P.G. Righetti, C. Gelfi, B. Verzola, L. Castelletti, Electrophoresis 22 (2001) 603611.

[33] J. Horvath, V. Dolník, Electrophoresis 22 (2001) 644-655.

[34] B. Verzola, C. Gelfi, P.G.Righetti, J. Chromatogr. A 868 (2000) 85-99.

[35] M.E. Legaz, M.M. Pedrosa, J. Chromatogr. A 719 (1996) 159-170.

[36] M.E. Lacey, A.G. Webb, J.V. Sweedler, Anal. Chem. 72 (2000) 4991-4998. 


\section{B.2. CAPILLARY ZONE ELECTROPHORESIS WITH A DYNAMIC DOUBLE COATING FOR ANALYSIS OF CARBOHYDRATE-DEFICIENT TRANSFERRIN IN HUMAN SERUM: PRECISION PERFORMANCE AND PATTERN RECOGNITION}

Published in: Journal of Chromatography A, 1013 (2003) 131 - 147 


\title{
CAPILLARY ZONE ELECTROPHORESIS WITH A DYNAMIC DOUBLE COATING FOR ANALYSIS OF CARBOHYDRATE-DEFICIENT TRANSFERRIN IN HUMAN SERUM: PRECISION PERFORMANCE AND PATTERN RECOGNITION
}

\author{
Christian Lanz ${ }^{1}$, Ulrich Marti ${ }^{2}$, Wolfgang Thormann ${ }^{1}$ \\ ${ }^{1}$ Department of Clinical Pharmacology, University of Bern, , \\ 3010 Bern, Switzerland. \\ ${ }^{2}$ Department of Clinical Chemistry, Inselspital, Bern, CH-3010 Bern, Switzerland.
}




\section{Abstract}

Capillary zone electrophoresis (CZE) with a dynamic double coating permits the simultaneous, individual, quantitative determination of transferrin (Tf) isoforms in human serum and thus carbohydrate deficient transferrin (CDT), the most specific marker available today for the detection of chronic, excessive alcohol intake. CZE of serum Tf was carefully evaluated using the P/ACE MDQ with fused-silica capillaries of $50 \mu \mathrm{m}$ I.D. and $60.2 \mathrm{~cm}$ total length, the CEofix CDT kit and the instrumental conditions recommended by the kit manufacturer. The precision performance assessed over a 20-day period according to the internationally accepted NCCLS EP5-A guidelines revealed the CZE assay as being highly reproducible with within-run and total precision being dependent on the $\mathrm{Tf}$ isoform level and RSD values ranging between 2.2 and $17.6 \%$. Inter-day RSD values for asialo-Tf were noted to be between 9.8 and $11.5 \%$ and for disialo-Tf between 3.8 and $8.6 \%$, whereas those for CDT levels of 0.87 and $4.31 \%$ of total Tf were determined to be 8.6 and $3.4 \%$, respectively. The RSD values for trisialo-Tf, tetrasialo-Tf, pentasialo-Tf and hexasialo-Tf were found to be between 0.4 and $4.1 \%$. Tf patterns are recognized and identified via detection times of $\mathrm{Tf}$ isoforms (intra-day and inter-day $\mathrm{RSD}$ values $<1.0 \%$ and $<1.7 \%$, respectively), immunosubtraction of $\mathrm{Tf}$ and enzymatic sequential cleavage of sialic acid residues. Furthermore, heterozygous $\mathrm{Tf} \mathrm{BC}$ and $\mathrm{Tf} \mathrm{CD}$ variants are assigned via spiking with a known mixture of $\mathrm{Tf}$ isoforms (e.g. the serum of a healthy $\mathrm{Tf} \mathrm{C}$ homozygote). Among the non-Tf peaks monitored, the CRP peak detected shortly before disialo-Tf was identified by immunosubtraction and peak magnitudes were found to correlate well with immunochemically determined CRP serum levels. The CZE assay with dynamic double coating could thereby be shown to be sensitive enough to determine elevated CRP levels in human serum. Furthermore, unusual peaks in the $\gamma$-region were identified by customary serum protein CZE, immunosubtraction CZE and immunofixation. 


\section{Introduction}

In the 1970s Stibler et al. observed an increase of transferrin (Tf) isoforms with isoelectric points $\geq 5.7$ in the serum and cerebrospinal fluid from patients with a chronic or repeated excessive alcohol intake [1-3]. Tf, the most important iron transporting protein, consists of 679 amino acids forming two iron binding sites and bears two Nlinked carbohydrate chains that are composed of neutral sugars and of zero up to eight end-standing, negatively charged sialic acid residues. The major $\mathrm{Tf}$ isoform, accounting for about $80 \%$ of whole $\mathrm{Tf}$ in human serum, contains four sialic acid residues and is known as tetrasialo-Tf with an isoelectric point of 5.4 [4,5]. Isoforms with pI values $\geq$ 5.7 encompass Tf molecules with two or less sialic acid residues and are therefore collectively named carbohydrate-deficient Tf (CDT). CDT is widely accepted as the most specific marker available today for the detection of chronic, excessive alcohol intake [6]. Different analytical approaches - based on isoelectric focusing, immunochemical determination of fractionated $\mathrm{Tf}$ isoforms and high performance liquid chromatography (HPLC) - have been employed for the investigation of Tf isoforms and the determination of CDT. Many of the developed methods are either time consuming or, due to possible interferences, prone to analytical inaccuracy [7,8]. During the last decade, capillary zone electrophoresis (CZE) has been extensively applied to the determination of $\mathrm{Tf}$ isoforms and thus also CDT. Among the various methods developed, CZE in dynamically coated fused-silica capillaries proved to be the most promising approach [8-16].

Dynamic capillary coatings are obtained with buffer additives that are being adsorbed onto the capillary via formation of a dynamic equilibrium. Macromolecule interactions with the capillary wall can thereby be prevented or at least minimized and the capillary is newly resurfaced for each analysis [17]. For the determination of CDT in human serum, our group recently compared three CZE methods with different dynamic capillary coatings, employing diaminobutane, spermine and a commercial double coating [9]. During that work, the double coating approach was identified as being the best. In this approach, the fused-silica capillary is first rinsed with an initiator buffer containing a polymeric polycation that is adsorbed to the wall surface followed by introduction of the running buffer containing a polymeric polyanion that is forming a second layer and thus providing the negative charge for a strong electroosmotic flow (EOF) towards the cathode. The CEofix CDT-kit of Analis (Namur, Belgium) comprises 
proprietary ionic polymers [18] that form a bilayer by noncovalent adsorption, such as the bilayers producedby Polybrene/dextran sulfate [19], poly(diallyldimethylammonium)/poly(styrene sulfonate) [20] and Polybrene/poly(vinylsulfonate) [21]. The EOF in these systems was determined to be essentially independent of $\mathrm{pH}$ and highly reproducible.

CZE of Tf in human serum allows the quantitative determination of CDT $[8,9,16]$. Moreover, it also provides information about the whole Tf pattern and other anionic serum compounds that are migrating slower than Tf and are thus detected ahead of Tf. Abnormalities in the Tf pattern [13,14] and the $\gamma$-region [15] can thereby be visualized also. There are currently major efforts being undertaken with the aim of a widespread adoption of the CZE assays for CDT into the routine arena, both for screening and confirmation in clinical and forensic analysis [8,9,14-16]. During the past year, the performance of the newly introduced commercial CZE kit for CDT (CEofix CDT-kit of Analis, Namur, Belgium) on the P/ACE MDQ capillary electrophoresis system of Beckman Coulter (Fullerton, CA, USA) was carefully evaluated using the instrumental conditions recommended by the kit manufacturer. We now wish to report (i) the precision performance data that were established according to internationally accepted NCCLS EP5-A guidelines [22], (ii) investigations of the nature of different abnormalities in the Tf pattern and the $\gamma$-region, and (iii) the identification of the $\mathrm{C}$ reactive protein (CRP) peak present in many sera in varying amounts and migrating directly in front of disialo-Tf.

\section{Experimental}

\subsection{Chemicals}

If not stated otherwise, chemicals were of analytical grade. Rabbit anti-human $\mathrm{Tf}$ antibody (titer: 2'800 mg/l) and rabbit anti-human C-reactive protein antibody (titer: 900 $\mathrm{mg} / \mathrm{l}$ ) were purchased from Dako (Glostrup, Denmark). Neuraminidase type $\mathrm{X}$ from clostridium perfringens (209 units/mg solid, 240 units/mg protein) and neuraminidase type III from vibrio cholerae ( $3 \mathrm{mg}$ protein $/ \mathrm{ml}, 3$ units $/ \mathrm{mg}$ protein) were obtained from Sigma (St. Louis, MO, USA). N-glycosidase F (100 units) was purchased from Roche Diagnostics (Rotkreuz, Switzerland). Buffers and reagents of the Analis CEofix-CDTkit for quantitation of CDT with the Beckman Coulter P/ACE MDQ (Kit No. 10004740) were kindly provided by Analis (Namur, Belgium). It is important to note that 
this kit comprises a buffer similar but not identical to that employed by Wuyts et al [14]. It is, however, believed to be the same as that now furnished for the Beckman Coulter $\mathrm{P} / \mathrm{ACE} 5500$ recently employed by Legros et al [16].

\subsection{Serum samples}

Patient sera were obtained from the departmental analytical laboratory where they were received for determination of CDT. Our own sera were used as sera of healthy persons. All blood samples, drawn by venipuncture, were collected in native plastic tubes without additives (Monovette, white cap, Sarstedt, Sevelen, Switzerland). After clotting, the blood samples were centrifuged at room temperature for $10 \mathrm{~min}$ at 2'000 g. The supernatant was transferred into $8.0 \mathrm{ml}$ polypropylene tubes. All sera were stored at $-20^{\circ} \mathrm{C}$ until use.

\subsection{Sample preparation}

Sera were slowly defrosted and vortex mixed at room temperature. According to the manufacturer's instructions, $60 \mu \mathrm{l}$ of serum and $60 \mu \mathrm{l}$ of the ferric solution of the kit (Analis) were mixed directly in the microtiter plate (Beckman Coulter, Fullerton, USA). The sample was mixed gently by aspiration and release of part of the fluid with a plastic pipette. No incubation time was required for iron saturation of the serum. For identification of $\mathrm{Tf}$ isoforms in the presence of genetic Tf variants, $30 \mu 1$ serum was combined with $30 \mu \mathrm{l}$ of a control serum of a healthy person prior to the addition of the $60 \mu \mathrm{l}$ of the ferric solution.

\subsection{Instrumentation, running conditions and data evaluation}

All CZE measurements and data evaluation were performed according to the instructions of the manufacturer of the CEofix CDT-kit (Analis). Briefly, a P/ACE MDQ capillary electrophoresis system (Beckman Coulter) that was equipped with a fused-silica capillary (Polymicro Technologies, Phoenix, AZ, USA) of $50 \mu \mathrm{m}$ I.D. and $60.2 \mathrm{~cm}(50.0 \mathrm{~cm}$ to the detector) total length was employed. The O.D. of the capillary was about $360 \mu \mathrm{m}$. For the evaluation of the precision performance capillaries with the same length and identical inner and outer diameter as described above were received from Analis. New capillaries were rinsed for 10 min with $0.2 \mathrm{M} \mathrm{NaOH}$ with a pressure of 40 psi applied from the inlet end. The same procedure was employed at the beginning 
of each day. At the end of a day the capillary was washed with $0.2 \mathrm{M} \mathrm{NaOH}$ and water ( 5 min each with 60 psi from the inlet to the outlet end). The capillary was stored wet and the ends were kept in water. Between runs the capillary was rinsed for 1 min with $0.2 \mathrm{M} \mathrm{NaOH}$ and a pressure of 60 psi from the inlet end and then with $0.2 \mathrm{M} \mathrm{NaOH}$ for 1.5 min by applying simultaneously a pressure of 20 psi at the inlet end and a constant current of $80 \mu \mathrm{A}$. At the beginning of a new run, the capillary was conditioned by applying a pressure of 15 psi from the inlet to the outlet side with the initiator buffer for $1.5 \mathrm{~min}$ followed by the run buffer of the CDT kit for $2 \mathrm{~min}$. The iron saturated sample was introduced from the microtiter plate by applying a vacuum of $0.3 \mathrm{psi}$ for $10 \mathrm{~s}$ at the outlet end. The temperature controls of the cartridge and sample tray were set to $40{ }^{\circ} \mathrm{C}$ and $15{ }^{\circ} \mathrm{C}$, respectively. The voltage applied was $28 \mathrm{kV}$ (anode at the inlet side) resulting in a current of about $26 \mu \mathrm{A}$ and a power level of about $1.21 \mathrm{~W} / \mathrm{m}$. UV detection was effected by using a $200 \mathrm{~nm}$ interference filter. Data registration, evaluation and storage were carried out with the 32 Karat Software, version 4.01 (Beckman). Data evaluation was based upon corrected peak areas of single isoforms (peak areas determined by valley-to-valley integration and divided by detection time) and are presented as area $\%$ in relation to the sum of the peak areas of all detected $\mathrm{Tf}$ isoforms. In contrast to the previous work from our laboratory [9], the amount of CDT was not evaluated as $\mathrm{Tf}$ index (area \% of disialo-Tf in relation to tetrasialo-Tf) but as area $\%$ of the sum of asialo-Tf and disialo-Tf in relation to the sum of the peak areas of all $\mathrm{Tf}$ isoforms.

\subsection{Evaluation of precision performance}

The precision performance of the Analis CEofix-CDT-kit was evaluated according to the EP5-A protocol from NCCLS [22]. A serum with a low level of CDT and one with a high amount of CDT, referred to as "sample low" and "sample high", respectively, and two corresponding controls referred to as "control low" and "control high", were analyzed over a period of twenty working days. The "sample low" was prepared from about $8 \mathrm{ml}$ serum of a healthy volunteer. The serum was homogenized and a total of $12060 \mu \mathrm{l}$ aliquots were stored in $0.5 \mathrm{ml}$ polypropylene vials at $-20{ }^{\circ} \mathrm{C}$ until use. The "control low" stemmed from another healthy person and was prepared in the same way. The "sample high" was prepared from 16 patient sera that were combined, vortex mixed, divided into 90 aliquots of $60 \mu \mathrm{l}$ each, and stored at $-20{ }^{\circ} \mathrm{C}$ until use. The "control high" was a serum pool of 4 patient samples and was stored as 45 aliquots of $60 \mu \mathrm{l}$ each. 
The evaluation was performed by one operator and started after the operator was familiar with the handling of all processes. Two separate sets of runs were analyzed on each day and the time period between the end of the first and beginning of the second set was at least two hours. Each sample in each set was analyzed in duplicates, resulting in a total of eight measurements per set or a total of 16 runs per day. For each set, the two aliquots of the "sample low", "sample high" and "control low" were prepared separately. For the "control high", one aliquot was prepared only and sample injection occurred twice from the same vial. The order of analysis was determined randomly for each set and the initiator and run buffers were changed every 100 analyses. All kits and reagents had the same lot number and capillaries from one lot were employed. Electropherograms were evaluated by valley-to-valley integration and the values of asialo-Tf and disialo-Tf were reported for all samples.

The values of the controls were reported on control charts including the mean value with two, three (warning limit) and four (out-of-control limit) standard deviations (SD). Every five days the mean value and the corresponding SD were recalculated of each chart from all acceptable data collected thus far. At day five, an additional set of runs was performed comprising twenty separately prepared aliquots of the "sample low". From these data a preliminary within-run precision was calculated according to the procedure stated in the protocol. All unexpected events and problems and their solutions were reported in detail. After collection of the data of 20 acceptable operating days, the within-run precision and the total precision of the CZE method for the determination of CDT was calculated according to the statistics described in detail in the protocol. In addition, relative standard deviations (RSD) for total precision of all four samples and controls, respectively, were calculated by dividing the SD by the corresponding mean value of the 80 measurements and multiplication with 100 .

\subsection{Immunosubtraction of Tf and CRP}

For immunosubtraction of $\mathrm{Tf}, 100$ to $400 \mu \mathrm{l}$ of serum was incubated in a plastic vial with varying relative volumes of anti-human $\mathrm{Tf}$ antibody. The smallest relative amount of anti-human Tf antibody was a quarter of the volume of the investigated serum, and the largest relative volume of antibody corresponded to four times the volume of serum. The sample was incubated at room temperature for $15 \min$ to $2 \mathrm{~h}$. Then, the incubate was centrifuged at $8000 \mathrm{~g}$ for $20 \mathrm{~min}$ at $4{ }^{\circ} \mathrm{C}$ and the supernatant was collected immediately and measured without further preparation. For data comparison 
with untreated sample, serum was equally diluted with water after iron saturation and analyzed under the same conditions. For immunosubtraction of CRP $60-200 \mu 1$ of serum was vortex mixed in a plastic vial with a volume of anti-human CRP antibody corresponding to $1 \%$ of the volume of the serum up to a volume of antibody that was twice the volume of the investigated serum. After incubation at room temperature for $0.5-2 \mathrm{~h}$, the sample was centrifuged at $8000 \mathrm{~g}$ for $20 \mathrm{~min}$ at $4{ }^{\circ} \mathrm{C}$. The supernatant was removed immediately and analyzed after saturation with an equal volume of the ferric solution of the CDT-kit (Analis). For some experiments the incubation of serum with anti-human CRP antibody was performed in the presence of the ferric solution. For investigation of the antibody solution, the reagent was diluted in water (2- to 20-fold) prior to analysis.

For sequential immunosubtraction of CRP and Tf in the same serum sample, 190 or $180 \mu \mathrm{l}$ of serum were incubated in a plastic vial with 10 or $20 \mu \mathrm{l}$, respectively, of anti-human CRP antibody at room temperature for $1 \mathrm{~h}$. Then, the precipitate was removed via centrifugation at $8000 \mathrm{~g}$ for $20 \mathrm{~min}$ at $4{ }^{\circ} \mathrm{C}$. $50 \mu \mathrm{l}$ of the collected supernatant was saturated with $50 \mu \mathrm{l}$ of the ferric solution of the kit (Analis) as described in section 2.3 and analyzed. The Tf immunosubtraction was performed with $60 \mu \mathrm{l}$ of the supernatant via addition of $120 \mu \mathrm{l}$ of the anti-human Tf antibody. The sample was incubated for $1 \mathrm{~h}$ at room temperature. After centrifugation for $20 \mathrm{~min}$ at $8000 \mathrm{~g}$ and $4{ }^{\circ} \mathrm{C}$, the supernatant was collected immediately and analyzed without further preparation.

\subsection{Digestion with neuraminidase and $\mathrm{N}$-glycosidase $\mathrm{F}$}

For digestion with neuraminidase type $\mathrm{X}$, the enzyme was reconstituted to 1 $\mathrm{U} / \mathrm{ml}$ with $50 \mathrm{mM}$ sodium acetate ( $\mathrm{pH} 5.0$, adjusted with acetic acid). $60 \mu \mathrm{l}$ of serum was saturated in a plastic vial with $60 \mu \mathrm{l}$ of the ferric solution of the CEofix CDT-kit and combined with $60 \mu \mathrm{l}$ of the enzyme solution. After vortex mixing, the sample was first incubated at $15^{\circ} \mathrm{C}$ for $20.5 \mathrm{~h}$ with the microtiter plate sitting in the garage of the $\mathrm{CE}$ instrument, then at $30^{\circ} \mathrm{C}$ for $2 \mathrm{~h}$ and then at $37^{\circ} \mathrm{C}$ for $6 \mathrm{~h}$. This sample was analyzed at various time points between 0 and $28.5 \mathrm{~h}$. Prior to the measurements at $26 \mathrm{~h}$ and 28.5 h, $90 \mu \mathrm{l}$ and $50 \mu \mathrm{l}$, respectively, of water was added to the sample. For digestion with neuraminidase type III, $360 \mu \mathrm{l}$ of serum was combined with $3 \mu \mathrm{l}$ of the purchased enzyme solution. After vortex mixing, the sample was first incubated at $37^{\circ} \mathrm{C}$ for $120 \mathrm{~h}$. 
For each analysis, a $30 \mu$ aliquot was withdrawn from the sample and combined with 30 $\mu \mathrm{l}$ of the ferric solution of the CEofix CDT-kit. For digestion with N-glycosidase F, 50 $\mu \mathrm{l}$ of serum was combined with $10 \mu \mathrm{l}$ of the reconstituted solution (100 U/100 $\mu \mathrm{l}$ water). After vortex mixing, the sample was first incubated at $37^{\circ} \mathrm{C}$ for $3.5 \mathrm{~h}$. Then, $40 \mu \mathrm{l}$ of the ferric solution was added and aliquots were analyzed at various time intervals during the following $24 \mathrm{~h}$ incubation at $37^{\circ} \mathrm{C}$.

\subsection{Routine assays for CRP, serum proteins and CDT}

CRP was determined in the routine laboratory with the CRP Tina-quant kit (Roche Diagnostics) on a Hitachi 917 Analyzer (Roche Diagnostics). CZE serum protein analyses and immunosubtraction CZE analyses were performed according to the manufacturer instructions using the Paragon CZE 2000 Clinical Capillary Electrophoresis System (Beckman, Brea, CA, USA) and the provided reagents (Beckman). Immunofixation analysis of serum proteins on agarose gels was performed with Hydrasis (Sebia, Issy-les-Moulineux, France). For other serum protein patterns obtained with these techniques refer to [23]. CDT of older sera were determined with the $\%$ CDT turbidimetric immunoassay (Bio-Rad, Hercules, CA, USA) whose CDT values < $6.0 \%$ were considered to be within the normal range. Newer sera were monitored with the \%CDT TIA turbidimetric immunoassay of Axis-Shield (Bio-Rad), an assay with a CDT cutoff value of $2.6 \%$.

\section{Results and discussion}

\subsection{Precision performance}

Analytical methods have to be investigated carefully before use in a routine arena and their capability of generating useful results has to be evaluated. They are fully described by their reliability criteria encompassing specificity, accuracy, precision and detectability, and their practicability criteria summarizing information about speed, cost, technical skill requirements and dependability. These criteria describe the performance characteristics of an analytical method [24]. In this manuscript, the precision performance, as an important part of the reliability of a method, of the commercially available CDT-kit is reported. It has been evaluated according to the NCCLS EP5-A guideline, an internationally accepted protocol that provides guidance and procedures 
for the evaluation of the precision of in vitro diagnostic devices. The variability of a device when used over a long period of time is of highest interest for the description of the precision performance. Several time-related components contribute to this total precision and can be characterized as within-run, between-run, within-day and day-today precision. The NCCLS document is designed to estimate the total precision and within-run precision [22]. Typical electropherograms for all four samples analyzed are presented in Fig. 1 and Table 1 summarizes the performance data obtained in our study. Standard deviations and the corresponding values of the degrees of freedom were calculated according to the protocol. Additionally, the mean of all data collected over the 20 day period $(n=80)$ was determined and used to calculate RSD values. Except for asialo-Tf of the "sample high", RSD values are shown to be below $10 \%$. For the withinrun precision and the total precision, RSD values were determined to be as low as 2.19 $\%$ and $3.57 \%$, respectively. For both, RSD values are becoming smaller as the isoform level is increasing. The detection limit for asialo-Tf and any other Tf isoform was estimated to be about $0.05 \%$ of total $\mathrm{Tf}$, whereas the limit of quantification was taken as $0.10 \%$ of total $\mathrm{Tf}$.

The precision performance evaluation according to the NCCLS EP5-A guideline is based upon duplicate analyses that are performed twice a day. Routine analyses are typically run once only which prompted us to evaluate the customary intra-day and inter-day precision data as well. The intra-day data correspond to the preliminary withinrun precision data obtained with the "sample low" on day five (for an electropherogram refer to Fig. 1A) and are summarized in the top line of Table 2. Corresponding data for the other $\mathrm{Tf}$ isoforms and the detection times for all isoforms are also included in Table 2. RSD values for detection times and isoform quantitation were found to be smaller than $1.0 \%$ and $5.1 \%$, respectively, values that correspond well to the preliminary data published previously [9]. Similar data were obtained for a sample with detectable asialoTf that was assessed outside the NCCLS EP5-A protocol (lower part of Table 2). Furthermore, the inter-day data presented in Table 3 are those obtained after evaluation of the results of the first aliquots of the four samples analyzed each day during the 20 day period. Again, RSD values for isoform quantitation were found to be dependent on peak size. For asialo-Tf, RSD was $<12 \%$ whereas for all other isoforms $<8.6 \%$. RSD values of detection times were determined to be $<1.7 \%$ in all cases (Table 4 ). For the quantitation of asialo-Tf and disialo-Tf, the values presented in Tables 2 and 3 were found to correspond well with those of Table 1. RSD values for CDT were determined to be between $5.07 \%$ and $8.55 \%$ for samples containing normal amounts of CDT. Corresponding values for samples with elevated CDT levels were between 1 and $4 \%$ 

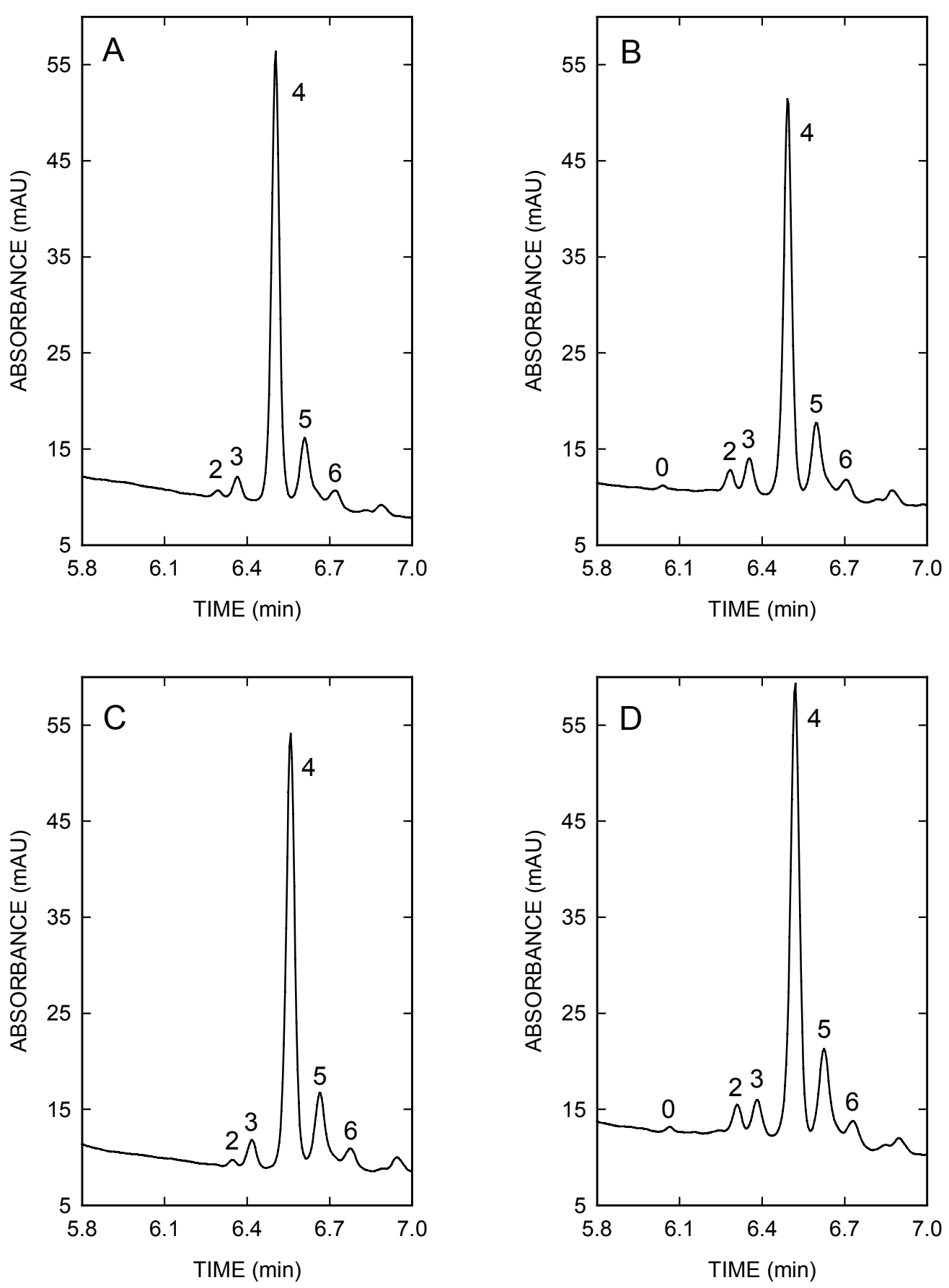

Figure 1: Typical CZE Tf patterns obtained with (A) "sample low", (B) "sample high", (C) "control low" and (D) "control high". Mean area\% values of the Tf isoforms are listed in Tables 1 and 3. Key: 0: asiaolo-Tf, 2: disialo-Tf, 3: trisialo-Tf, 4: tetrasialo-Tf, 5: pentasialo-Tf, 6: hexasialo- 


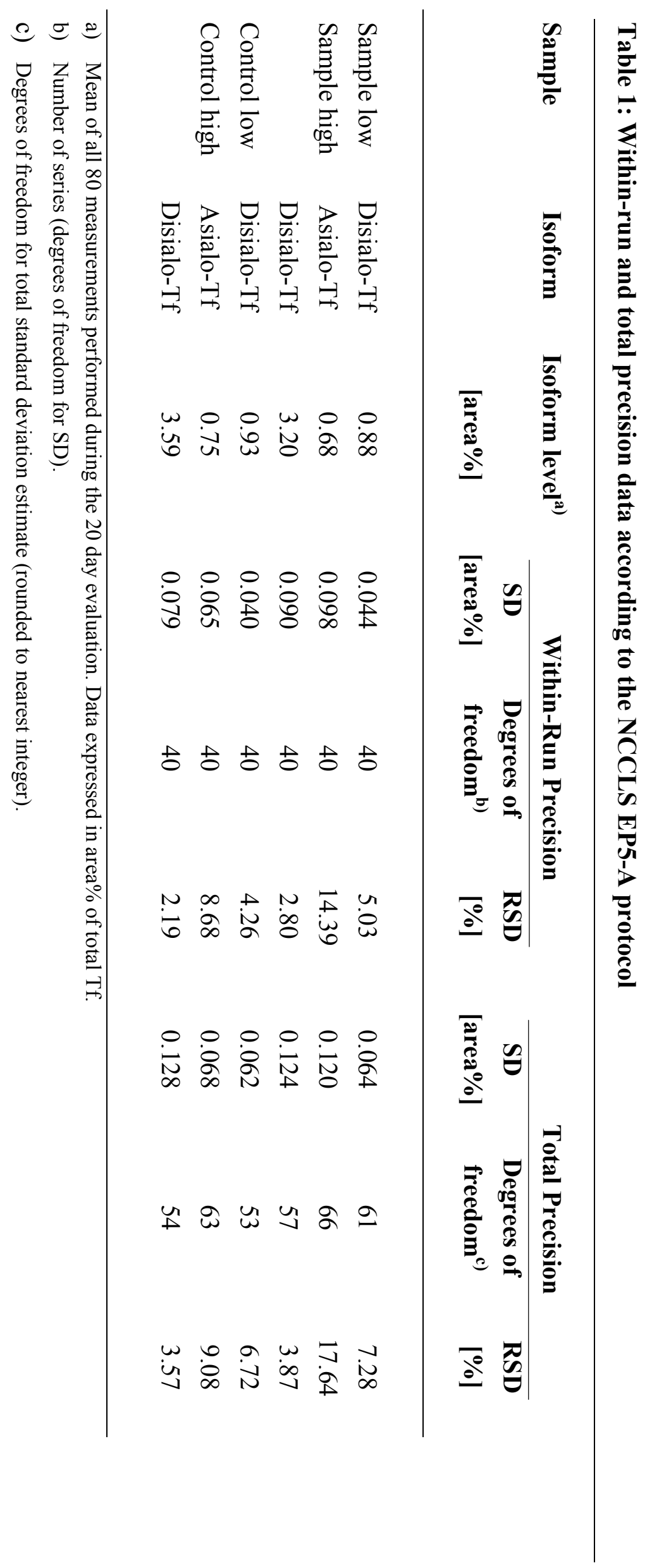




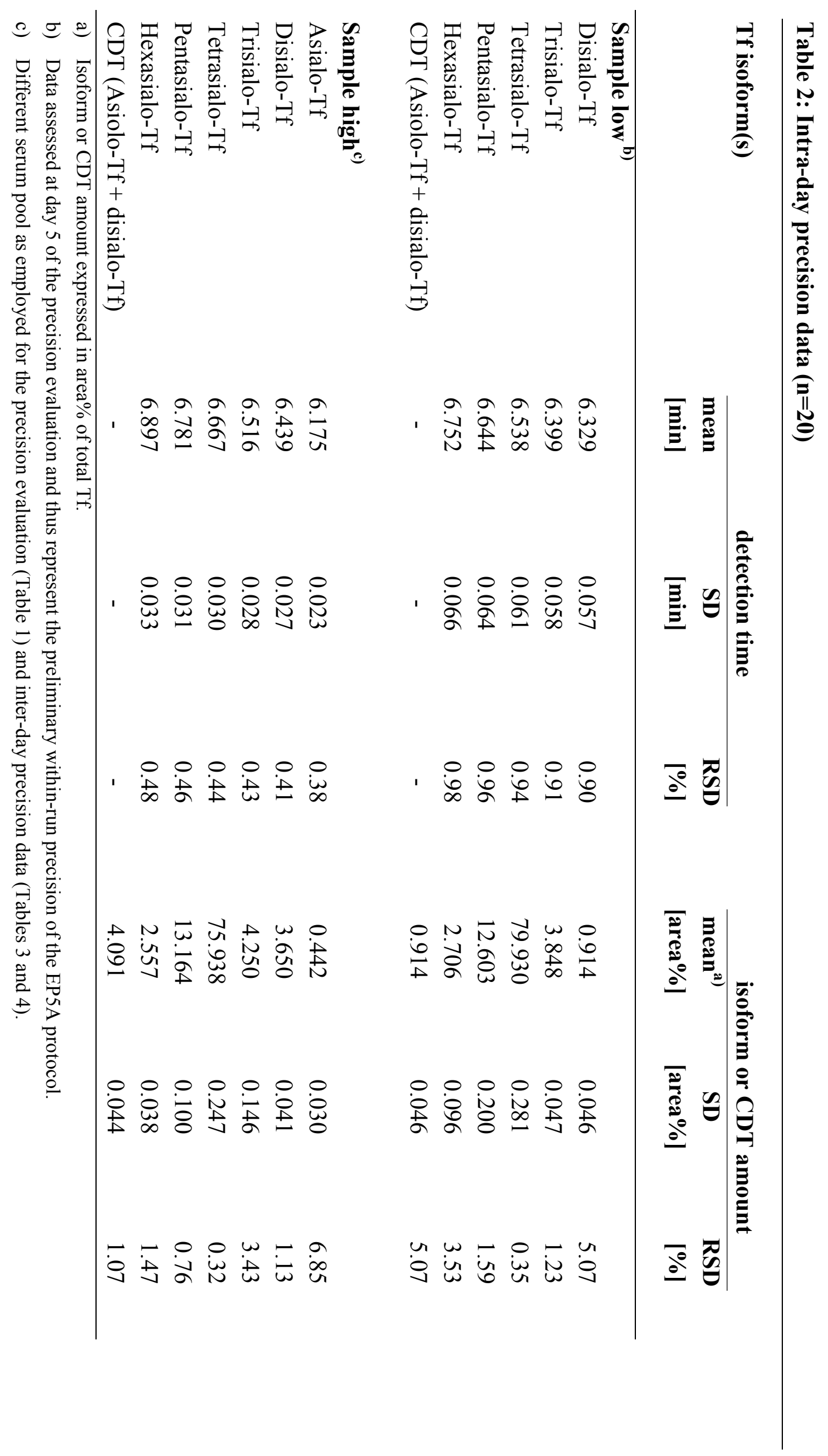




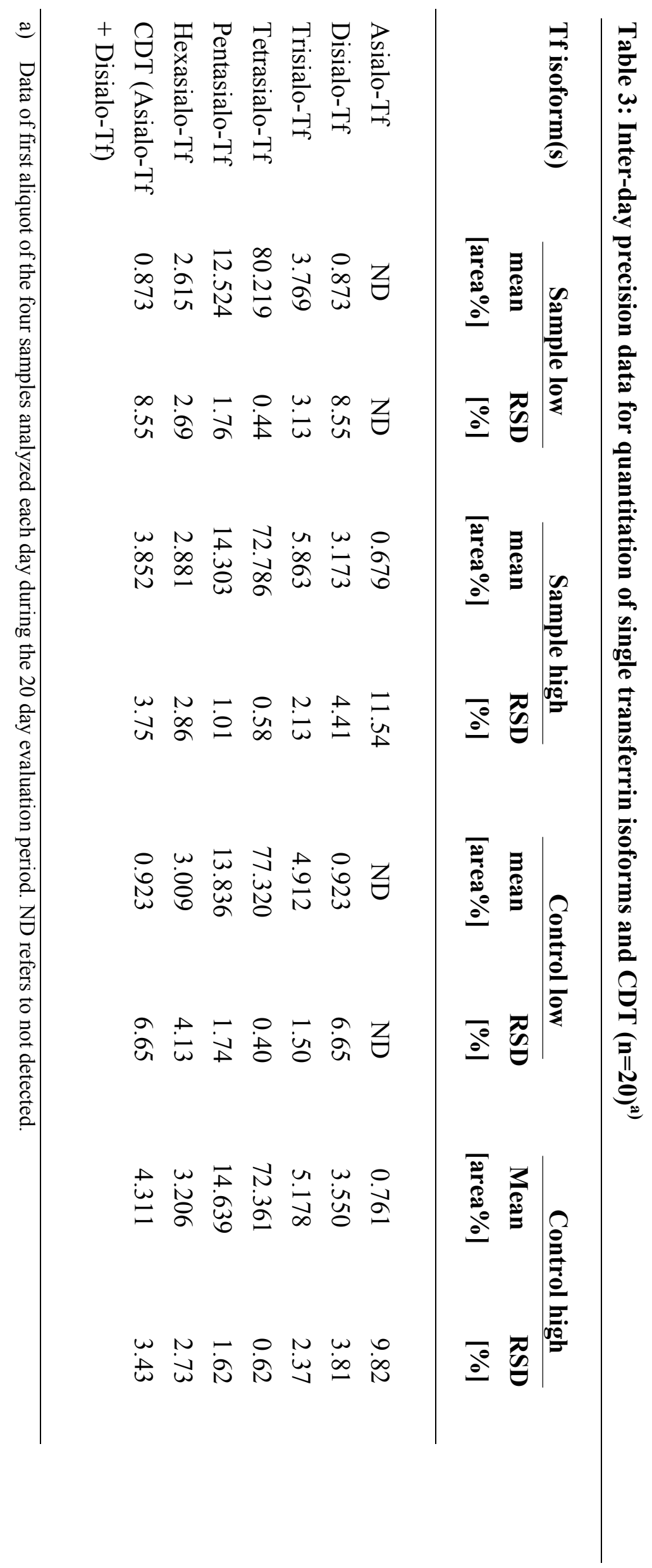




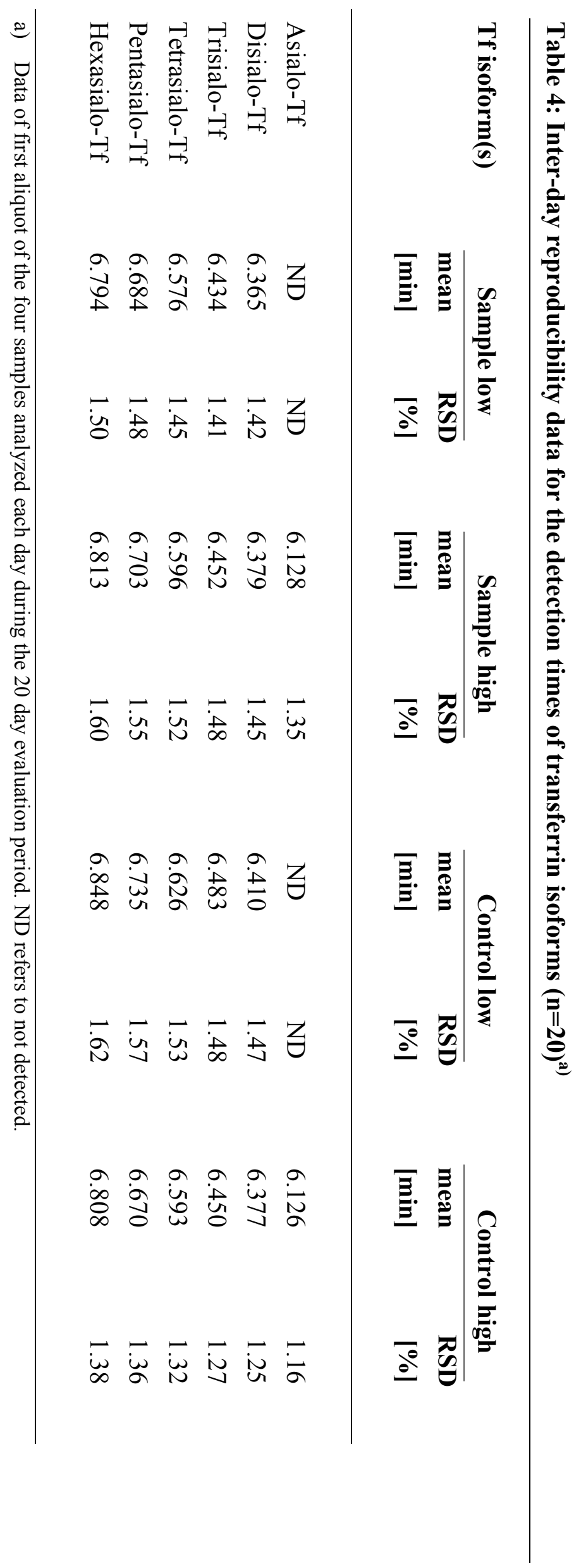


(Tables 2 and 3). These data indicate that the CE method is highly reproducible and precise.

During the execution of the precision performance study, reagents were changed on days 5,11 and 17 and the data of all 20 days were found to be acceptable. The study, i.e. the stability of the system, was surveyed with control charts that included the mean value with two, three (warning limit) and four (out-of-control limit) SDs and every five days the mean value and the corresponding SD were recalculated of each chart from all acceptable data collected thus far. The study, however, could not be carried out without unexpected events. A total of three capillaries were used. Due to sticking of the cap of the waste vial to the interface block, the capillary broke on days 3 and 9 and had to be replaced. Mechanical problems with the sample garage led to the restart of sequences, namely on days 11 (three times), 12 (once), 13 (once), 17 (twice), 18 (once), 19 (once) and 20 (twice). The garage was replaced after completion of the study. There was a failure of current during the analysis of three samples, a current leakage during the analysis of one sample and higher detection times of the $\mathrm{Tf}$ isoforms for one sample. These five samples were reanalyzed at the end of the sequence, i.e. the same day. Furthermore, isoform values were somewhat lower than usual on day 16 (the values of the two controls were between $-2 \mathrm{SD}$ and $-4 \mathrm{SD}$ ), but no intervention was necessary.

\subsection{Recognition and identification of the transferrin pattern}

Recognition and identification of the peaks in the electropherograms that originate from $\mathrm{Tf}$ isoforms can be accomplished via assessment of the relative migration times of the $\mathrm{Tf}$ isoforms (particularly in relation to tetrasialo-Tf), analysis of the sample prior and after spiking with a known mixture of $\mathrm{Tf}$ isoforms and/or prior and after immunosubtraction of $\mathrm{Tf}$, and enzymatic sequential cleavage of sialic acid residues with analysis of the whole range of samples $[9,14,16]$. Detections times of Tf isoforms were found to vary within a very small range only (Tables 2 and 4, [9,16]) and can thus typically be employed for peak assignment. Unambiguous recognition of Tf peaks was accomplished via immunosubtraction in free solution using an anti-human Tf antibody. For that purpose, the ratio of the volumes of serum and antibody solution and the incubation conditions had to be optimized. A relative amount of antibody solution that was $25 \%$ of the investigated serum was found to be insufficient for a complete removal of the Tf isoforms. Small peaks of tetrasialo- and pentasialo-Tf were detected (Fig. 2A, 
fourth electropherogram from top). The same was true employing equal amounts of antibody solution and serum (Fig. 2A, third electropherogram from top). Complete immunosubtraction of Tf isoforms could be obtained using $80 \mu \mathrm{l}$ of serum and $160 \mu \mathrm{l}$ of antibody solution (Fig. 2A, second graph from the top) whereas a further increase of the relative amount of antibody solution did not provide any advantage (Fig. 2A, top electropherogram). To the contrary, higher amounts of antibody solutions led to electropherograms that were overloaded with $\gamma$-globulins. The impact of the incubation
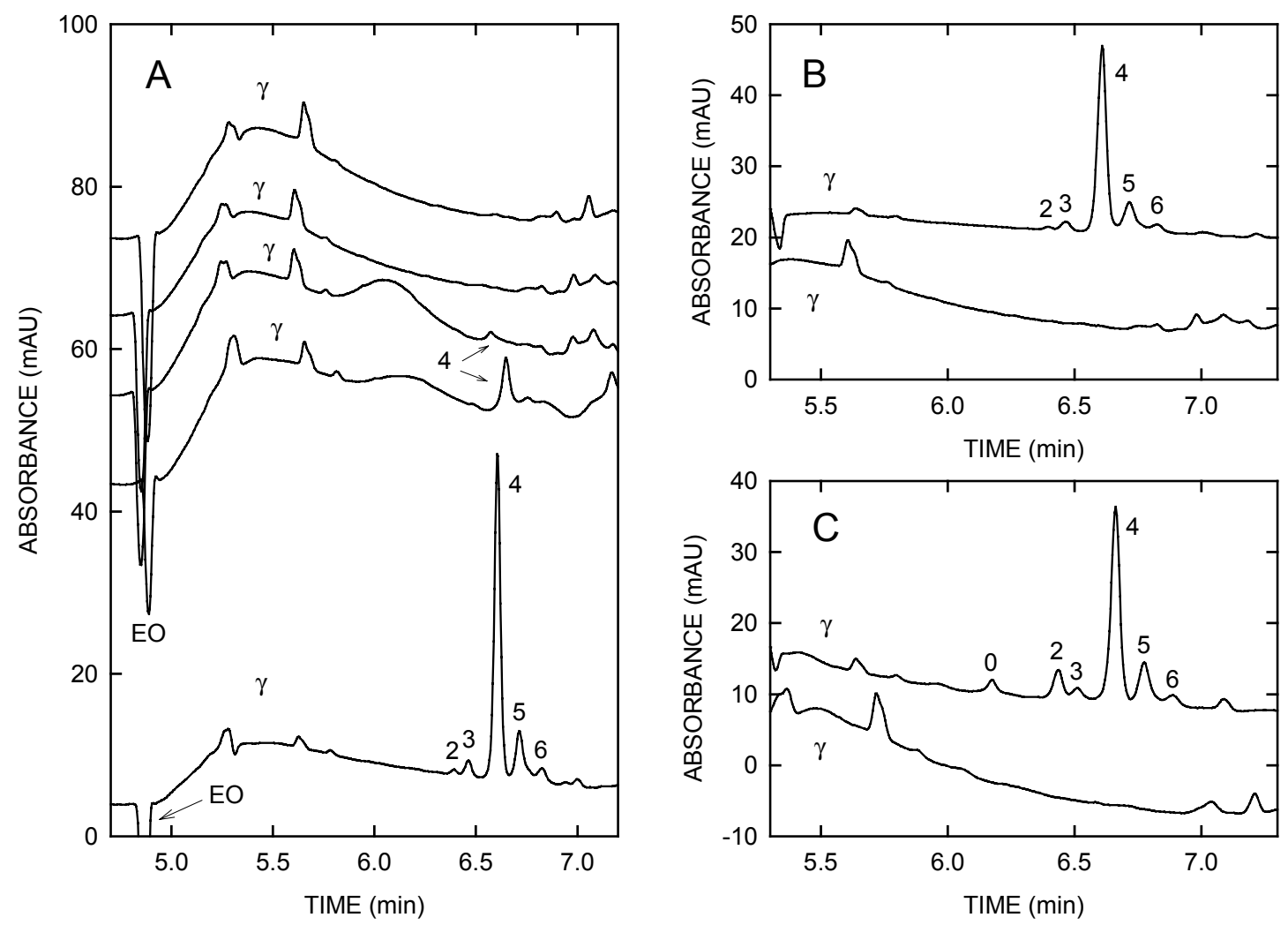

Figure 2: Immunosubtraction of Tf. A) Electropherogram obtained for a serum of a healthy individual without Tf antibody pretreatment (bottom graph) and after immunosubtraction using the serum/antibody solution proportions of $1: 4,1: 2,1: 1$ and $4: 1(\mathrm{v} / \mathrm{v}$, from top to center, electropherograms plotted with different $y$-axis offsets). $\gamma$ refers to the gamma region and EO marks the electroosmotic flow. B) Electropherogram of a three-fold diluted serum (top graph, presented with a y-axis shift of $7 \mathrm{mAU}$; serum dilution with two aliquots of ferric solution) of a healthy subject (CZE CDT value of $0.88 \%$ ) compared to the data obtained after immunosubtraction of $\mathrm{Tf}$ with the reagent volume being twice that of the serum (bottom graph). C) Electropherogram of an alcohol abuser (CZE CDT value of $13.3 \%$, presented with a $\mathrm{y}$-axis shift of $20 \mathrm{mAU}$ ) together with data obtained after immunosubtraction of $\mathrm{Tf}$ and the experimental conditions as for panel B. The numbers of all graphs refer to the detected Tf isoforms (for key see Fig. 1). 
time interval was studied with the optimized serum/antibody solution ratio. An incubation time of $1 \mathrm{~h}$ was found to be sufficient. No improvement was obtained with longer incubation times. However, an incubation of $15 \mathrm{~min}$ led to incomplete immunosubtraction (data not shown). Thus, Tf isoforms were immunosubtracted by having twice the volume of antibody solution compared to serum, an incubation at room temperature for $1 \mathrm{~h}$ and centrifugation for $20 \mathrm{~min}$ at $8000 \mathrm{~g}$ and $4{ }^{\circ} \mathrm{C}$. Fig. $2 \mathrm{~B}$ and $\mathrm{C}$ depict electropherograms obtained before and after immunosubtraction of $\mathrm{Tf}$ from the sera of a healthy subject and an alcohol abuser, respectively. The identification of the peak labeled as hexasialo-Tf was found to be somewhat difficult. In some sera the possibility of an interference in this part of the beta-region cannot be excluded (see Fig. 2B, bottom graph).

As has been discussed previously for the P/ACE $5500[14,16]$ and also briefly for the P/ACE MDQ [9], neuramidase can be employed for the enzymatic cleavage of the terminal sialic acid residues of Tf. During hydrolysis, the higher sialylated Tf isoforms are gradually disappearing with time and new peaks are arising around and before the position of asialo-Tf as well as between asialo- and disialo-Tf (Fig. 3). The transient peak marked with "a" is presumably monosialo-Tf. It is shown to gradually increase within the first $20 \mathrm{~h}$ and $24 \mathrm{~h}$ for the cases of Figs. 3A and 3B, respectively. Then this peak is decreasing and finally vanishing. Peaks " $b$ " and " $c$ " are assumed to be glycosylated and asialylated Tf isoforms. All these peaks can be immunosubtracted using an antibody against $\mathrm{Tf}$ and the patterns observed in our work are comparable to those reported by Legros et al. [16]. Asialo-Tf is not affected by the neuraminidase hydrolysis. This is nicely illustrated with the $0.33 \mathrm{~h}$ to $2.66 \mathrm{~h}$ time points of Fig. $3 \mathrm{~B}$. According to our data, asialo-Tf gradually becomes part of the growing peak " $b$ ". It is, however, not identical to the isoform of peak b. Using N-glycosidase F to cleave the glycans present in the isoforms of Tf, including those of peaks " $b$ " and "c", and thereby producing asialo-Tf did not yet provide the anticipated data. Incubation of serum resulted in small amounts of asiolo-Tf being formed only. Further efforts are required to investigate the processes leading to this final proof.

The screening of about 200 sera revealed five electropherograms with two major and a total of more than six Tf peaks (center graphs of Fig. 4), i.e. patterns that are clearly different compared to those normally observed with the sera of healthy subjects or alcohol abusers (Figs. 1, 2B and 2C). The area and heights of the two major peaks detected were found to be about half of the area and height of the tetrasialo-Tf peak of a normal serum pattern, thus supporting the hypothesis that the observed abnormal patterns belonged to genetic $\mathrm{Tf}$ variants. Human $\mathrm{Tf}$ is known to show genetic 
polymorphism caused by the substitution of one or more amino acids in the primary structure of the protein. Up to now, at least 38 genetic Tf variants could be identified and only four of these occur with a prevalence of $>1 \%$. Tf variants can be divided into three groups. Tf-C, the most common phenotype in all populations, encompasses 16 distinct subtypes with slightly different isoelectric points, Tf-C1 being that with the highest prevelance $(>95 \%)$ in Caucasians. Tf-B variants with lower pI values than Tf-C occur with lower frequencies, whereas genetic Tf-D variants having higher $\mathrm{pI}$ values are reported to be rare in Caucasians, but not in some other populations [6,25-27]. Closer inspection of the monitored electropherograms revealed the overall pattern as being a
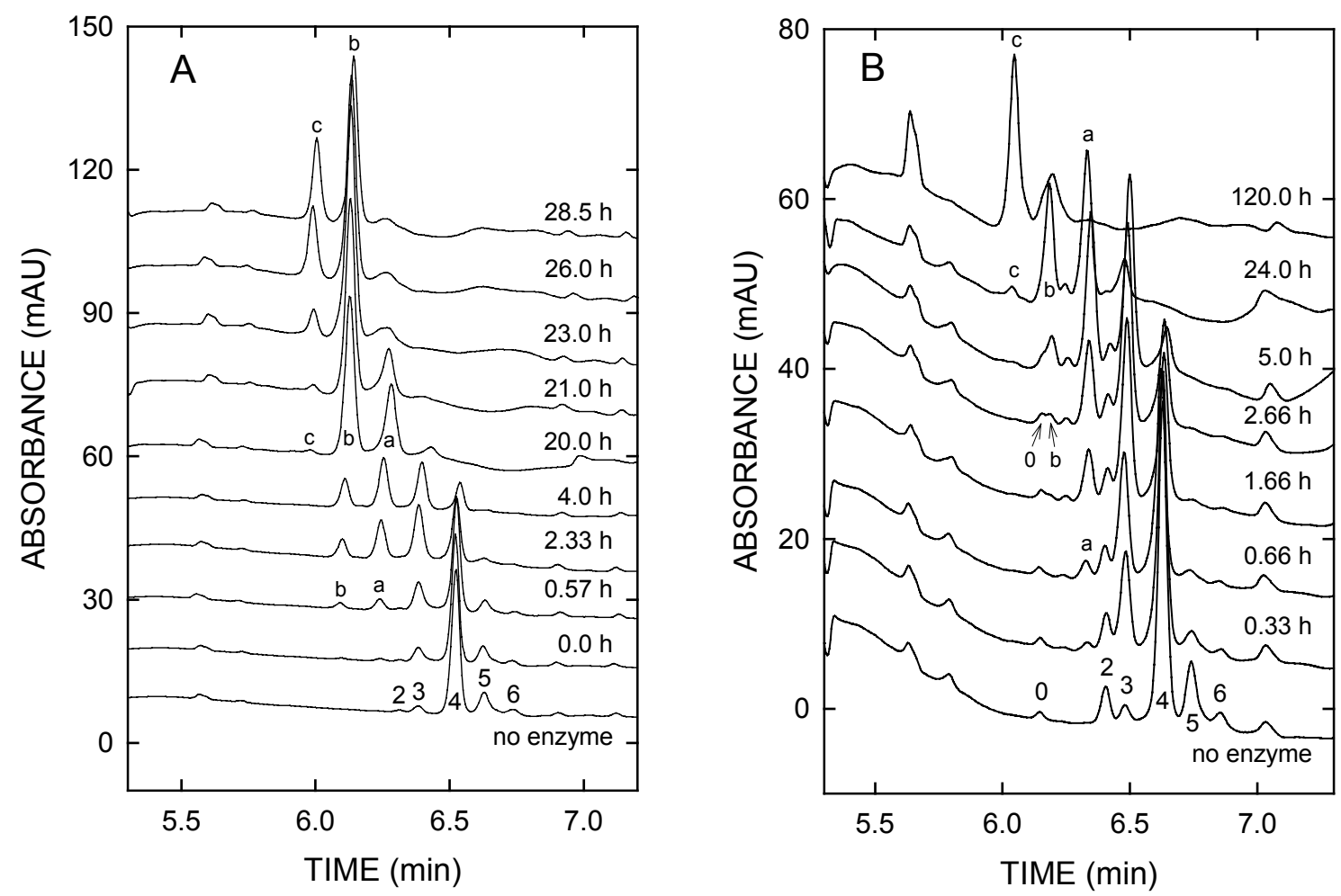

Figure 3: Electropherograms obtained after enzymatic sequential cleavage of sialic acid residues from sera of A) a healthy subject with a CZE CDT level of $0.76 \%$ and B) an alcohol abuser with a CZE CDT value of $8.91 \%$ using A) neuraminidase type $\mathrm{X}$ and B) neuraminidase type III. The bottom graphs depict the patterns obtained without enzyme using the same amount of water instead of the enzyme solution. Consecutive elecropherograms monitored at the indicated time intervals after commencement of the enzymatic incubation are presented with $y$-axis offsets of the $\mathrm{x}$-axis adjusted for equal appearance of the corresponding peaks. The numbers of the bottom graphs refer to the detected Tf isoforms (for key see Fig. 1). 
composite of two Tf patterns with an anodic time shift (shift to the right, center graph of Fig. 4A) or with a cathodic time shift (shift to the left, center graph of Fig. 4B). Immunosubtraction with the anti-human $\mathrm{Tf}$ antibody led to electropherograms with no peaks in the $\beta$-region (bottom graphs of Fig. 4) thereby proving the Tf origin of all the monitored peaks. Furthermore, in order to distinguish between the Tf isoforms normally observed and those shifted to the right or to the left, the sera were spiked with the control serum of a healthy individual and reanalyzed (top graphs of Fig. 4). B and D Tf variants could thereby be identified. In four of the five cases, the major peak of the atypical $\mathrm{Tf}$ isoforms, considered to be tetrasialo-Tf, eluted about $7 \mathrm{~s}$ after the corresponding peak of the common C-tetrasialo-Tf, thus indicating a shift to the anodic side due to an increase of the electrophoretic mobility (decrease of $\mathrm{pI}$ ) of the unexpected isoforms (Fig. 4A, top graph) and the presence of genetic Tf-B variants in these cases originating from Tf-BC heterozygote individuals. All peaks present in the
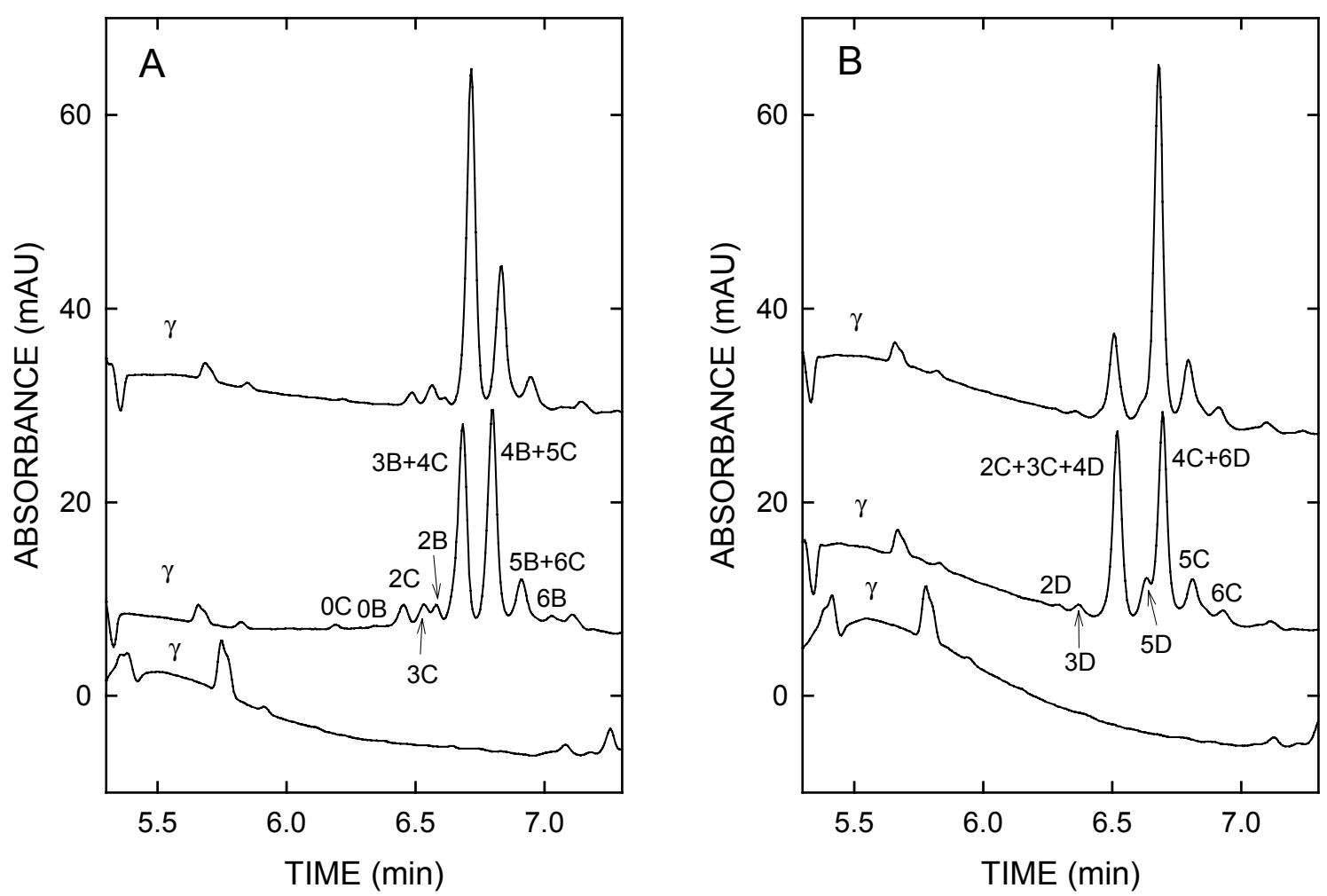

Figure 4: Electropherograms obtained for A) a heterozygous Tf-BC variant and B) a heterozygous Tf-CD variant monitored without pretreatment (center graphs), after immunosubtraction of Tf (bottom graphs) and after spiking with the serum of a healthy individual (top graphs). Center and top graphs are presented with y-axis offsets of A) $8 \mathrm{mAU}$ and $27 \mathrm{mAU}$, respectively, and B) $7 \mathrm{mAU}$ and $27 \mathrm{mAU}$, respectively. 
electropherograms could beunambiguously assigned either to a single isoform of one of the two genetic Tf alleles (B- or C-Tf variant) or to comigrating isoforms of the two Tf variants (Fig. 4A). In one case, a shift of the unusual Tf isoforms of about $10 \mathrm{~s}$ to the cathodic side was observed (top graph of Fig. 4B). Tf isoforms with shorter migration times compared to those of the common $\mathrm{Tf}-\mathrm{C}$ can be attributed to the presence of a rare genetic Tf-D variant with isoforms having higher isoelectric points. All peaks could be allocated to single or unresolved isoforms of the Tf-C and Tf-D alleles (Fig. 4B). Although the number of selected individuals in our investigation is too small and heterogeneous to report the prevalence of Tf-BC (about $2 \%$ ) and Tf-CD (about $0.5 \%$ ) heterozygotes, it can be stated that the occurrence of Tf variants is not negligible. These findings are in agreement with the literature [6,26,27] and of importance for the determination of CDT for identification of alcohol abusers.

In [27] it was shown that $\mathrm{BC}$ and $\mathrm{CD}$ heterozygotes regularly led to low and high CDT results, respectively, when analyzed by any of the commercially available immunoassays, with the consecutive possibility of incorrect classification and misinterpretation of the test result. This is also true for the two samples depicted in Figs. $4 \mathrm{~A}$ and $4 \mathrm{~B}$ for which the \%CDT TIA assay revealed CDT values of $4.6 \%$ and $2.9 \%$, respectively. These values are higher than the reference value of $2.6 \%$. Using $\mathrm{CZE}$, the elevated CDT value of the serum of Fig. 4A could be confirmed. The CZE CDT level was estimated to be $8.28 \%$, a value that was composed of the peaks of $0 \mathrm{C}$ and $0 \mathrm{~B}$ and twice the peak $2 \mathrm{C}(0 \mathrm{C}+0 \mathrm{~B}+2 \times 2 \mathrm{C})$. For the serum of Fig. $4 \mathrm{~B}$, however, the $\mathrm{CZE}$ CDT value of $0.84 \%$ (estimated to represent twice the peak value of $2 \mathrm{D}$ ) is clearly comparable to those of individuals without elevated CDT levels (Figs. 1,2,3). In analogy to HPLC [27], CZE is thereby shown to permit proper classification of the CDT patterns of $\mathrm{BC}$ and $\mathrm{CD}$ heterozygotes alike.

\subsection{Recognition and identification of non-transferrin peaks}

From the about 200 sera analyzed, selected electropherograms were found to comprise a peak of varying magnitude that was detected just prior to disialo-Tf (Fig. $5 \mathrm{~A}$ ). Immunosubtraction of $\mathrm{Tf}$ isoforms and reanalysis revealed that this peak did not belong to Tf (Fig. 5B, second graph from bottom). The manufacturer of the CEofix CDT-kit indicated that CRP could be responsible for that peak. Thus, immunosubtraction experiments with an anti-human CRP antibody were performed. In a 
first approach, $135 \mu \mathrm{l}$ serum containing a large amount of CRP $(120 \mathrm{mg} / \mathrm{l}$, top graph of Fig. 5B) was incubated with $15 \mu \mathrm{l}$ anti-CRP antibody solution for $30 \mathrm{~min}$ at room temperature. After centrifugation, $60 \mu \mathrm{l}$ supernatant was saturated with $60 \mu \mathrm{l}$ of ferric solution. Analysis of this sample revealed a decreased peak of interest and an increase of the peak of disialo-Tf (Fig. 5B, second graph from top). The cause for the latter fact could be revealed as originating from a component in the anti-CRP antibody solution that was found to comigrate with disialo-Tf. The use of a higher amount of antibody solution led to a complete removal of the CRP peak together with an increased interference with disialo-Tf. For sera with smaller amounts of CRP, $5 \%$ (v/v) antibody solution was found to provide a complete removal, whereas with $2 \%(\mathrm{v} / \mathrm{v})$ or less
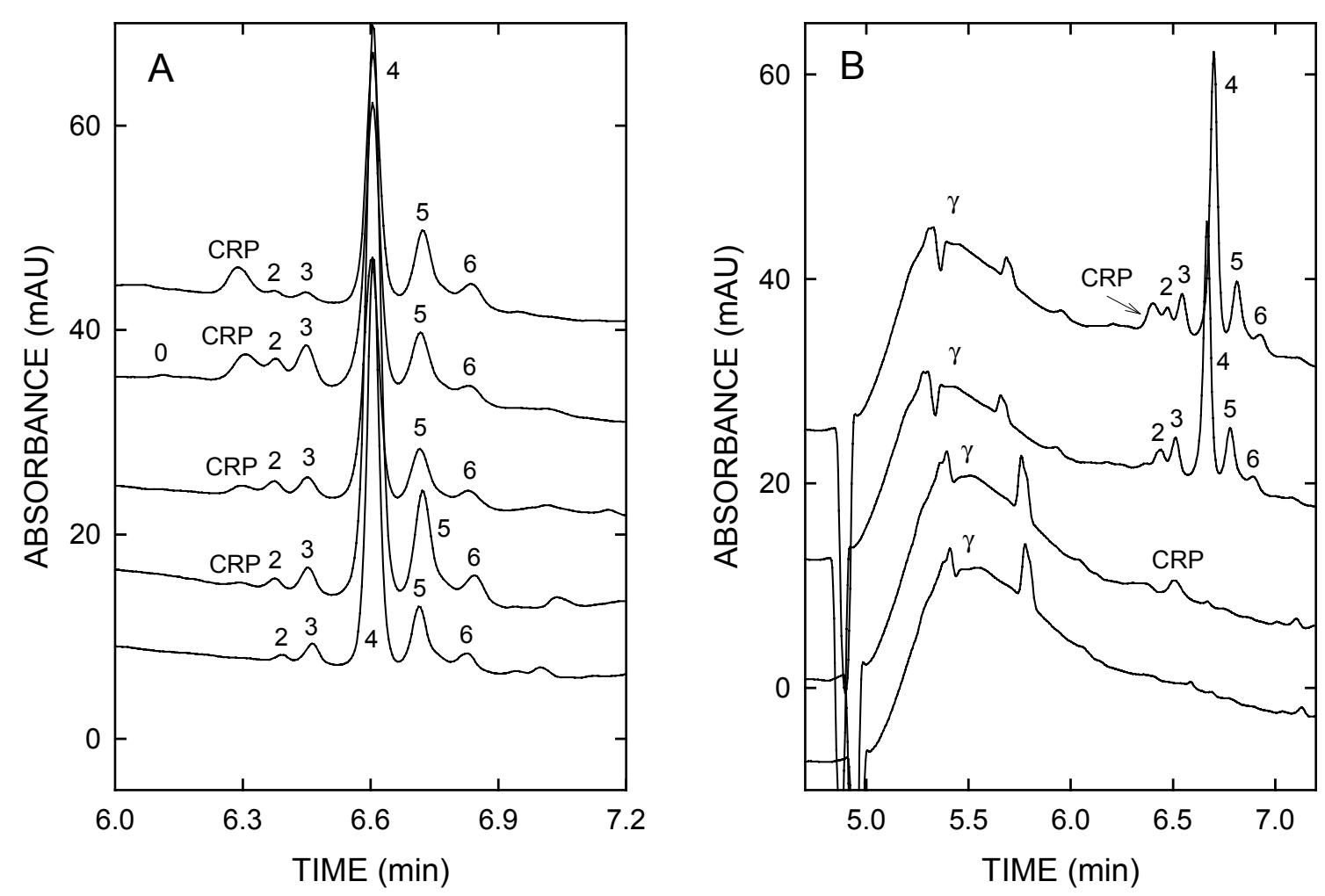

Figure 5: Electropherograms before and after immunosubtraction of CRP. A) Patterns of sera having undetectable CRP, $10 \mathrm{mg} / 1,34 \mathrm{mg} / \mathrm{l}, 120 \mathrm{mg} / \mathrm{l}$ and $141 \mathrm{mg} / \mathrm{l} \mathrm{CRP} \mathrm{(from} \mathrm{bottom} \mathrm{to} \mathrm{top,} \mathrm{respectively;} \mathrm{data}$ presented with $\mathrm{y}$-axis offsets of $\mathrm{x}$-axis adjusted for equal appearance of tetrasialo-Tf). B) Electropherograms of a patient serum containing $120 \mathrm{mg} / \mathrm{l} \mathrm{CRP}$, of the same serum after immunosubtraction of CRP using $10 \%$ antibody solution, of the same serum after immunosubtraction of Tf, and of the same serum after immunosubtraction of CRP and Tf (from top to bottom, respectively; data presented with y-axis offsets). The CRP values are those determined with the immunoassay. 
insufficient immunosubtraction of CRP was obtained. No improvement was noted withincubation times longer than $1 \mathrm{~h}$ and by incubating the serum with anti-CRP antibody in the presence of iron (data not shown). A procedure for the sequential removal of CRP and Tf isoforms in the same serum was developed. In the first step, CRP was removed employing 5 or $10 \%(\mathrm{v} / \mathrm{v})$ of the anti-human CRP antibody solution, depending on the amount of CRP present in the serum. One part of the supernatant (50 $\mu 1$ ) was saturated with an equal amount of ferric solution and analyzed (Fig. 5B, second graph from top). The other part of the supernatant $(60 \mu \mathrm{l})$ was used for immunosubtraction of $\mathrm{Tf}$ (as described in section 2.6). After sequential immunosubtraction, CRP and Tf were no longer detected (Fig. 5B, bottom graph).

The CRP peaks in the electropherograms amounted up to 5756 area units (top graph of Fig. 5A). The CRP level in the four patient sera of Fig. 5A and 14 other sera was determined using a commercial immunoassay with a detection limit of $3 \mathrm{mg} / \mathrm{l}$ (normal CRP range: $<5 \mathrm{mg} / \mathrm{l}$ ) and revealed CRP levels between 4 and $141 \mathrm{mg} / \mathrm{l}$. Regression analysis of the peak areas in the electropherograms (between 433 and 5756 area units) with the immunoassay data resulted in a linear relationship (y $=289.1+$ $43.5 \mathrm{x}$ where $\mathrm{x}$ represents the immunoassay CRP data) with the correlation coefficient $r$ being 0.962. Furthermore, for four sera whose CRP levels could not be determined with the immunoassay (CRP $<3 \mathrm{mg} / \mathrm{l}$ ), small peaks (peak areas: 176-831) were detected in the electropherograms. Thus, the CZE assay appears to be sensitive enough to determine elevated CRP levels in human serum. Based on our current experience, CRP levels up to about $70 \mathrm{mg} / \mathrm{l}$ were found not to interfere with CDT determination. With higher values, resolution between CRP and disialo-Tf was noted to become $<1.24$ and thus somewhat hampered the exact determination of disialo-Tf. Nevertheless, for the data presented in Fig. 5A, classification by CZE revealed data that were in agreement with those assessed by the immunoassays. Elevated CDT values were noted for the sera containing $34 \mathrm{mg} / \mathrm{l}$ and $120 \mathrm{mg} / \mathrm{l} \mathrm{CRP} \mathrm{(CZE} \mathrm{CDT} \mathrm{values} \mathrm{of} 2.36 \%$ and $2.06 \%$, respectively, and \%CDT values with the older immunoassay of $9.1 \%$ and $8.0 \%$, respectively) and normal CDT values were obtained for the sera with $10 \mathrm{mg} / \mathrm{l}$ and $141 \mathrm{mg} / \mathrm{l} \mathrm{CRP} \mathrm{(CZE} \mathrm{CDT} \mathrm{values} \mathrm{of}$ $1.03 \%$ and $0.50 \%$, respectively, and \%CDT values with the newer immunoassay of 1.4 $\%$ and $1.9 \%$, respectively).

Four patient sera analyzed with the CZE assay revealed an abnormal pattern in the $\gamma$-region in that a large peak (15 to $35 \mathrm{mAU}$ ) was detected in the time window between about 5.2 to $6.0 \mathrm{~min}$ (Fig. 6). In one case, the unexpected peak was interfering 

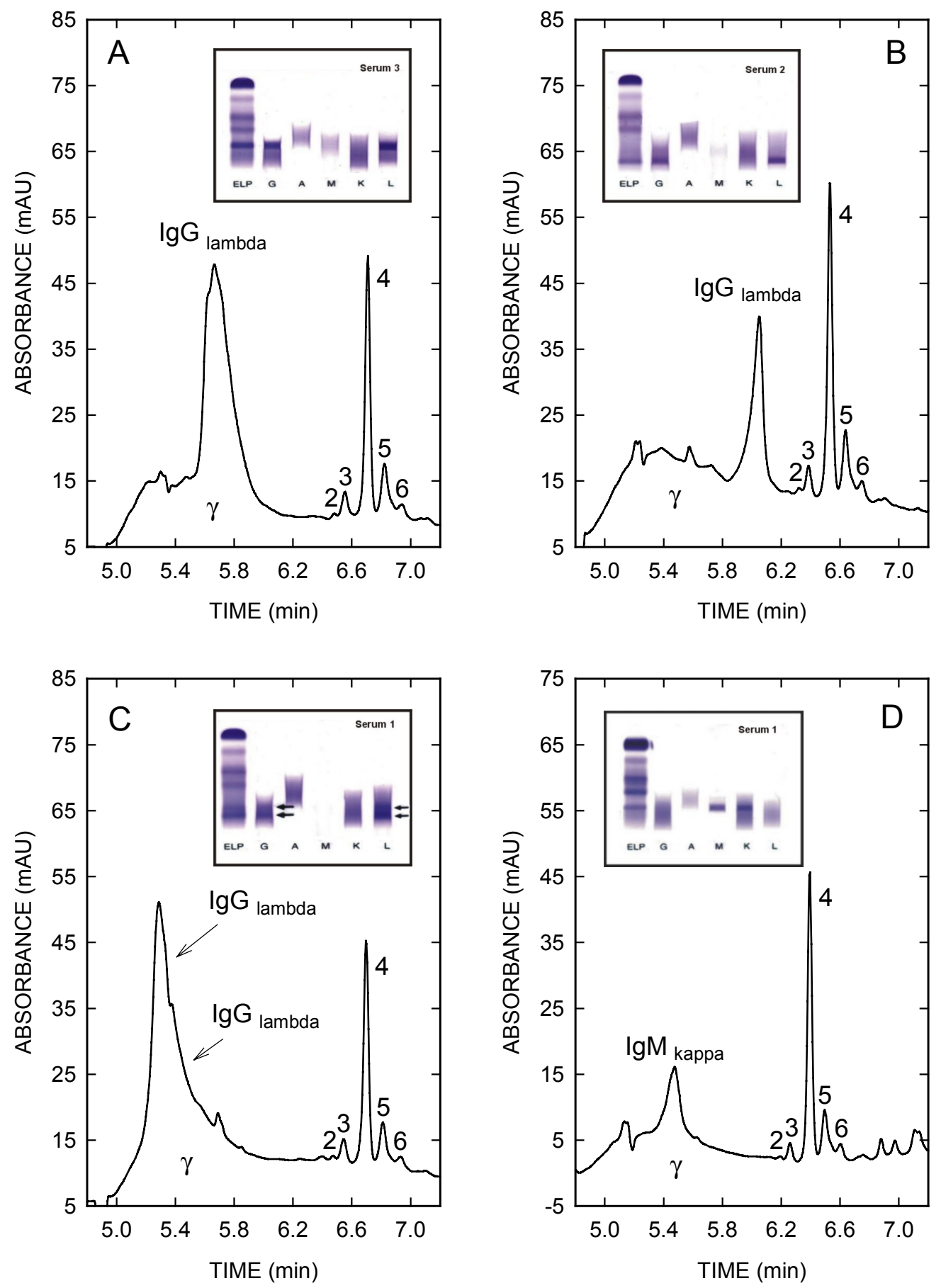

Figure 6: Electropherograms with large, unusual peaks in the $\gamma$-region. The insets depict the immunofixation data. 

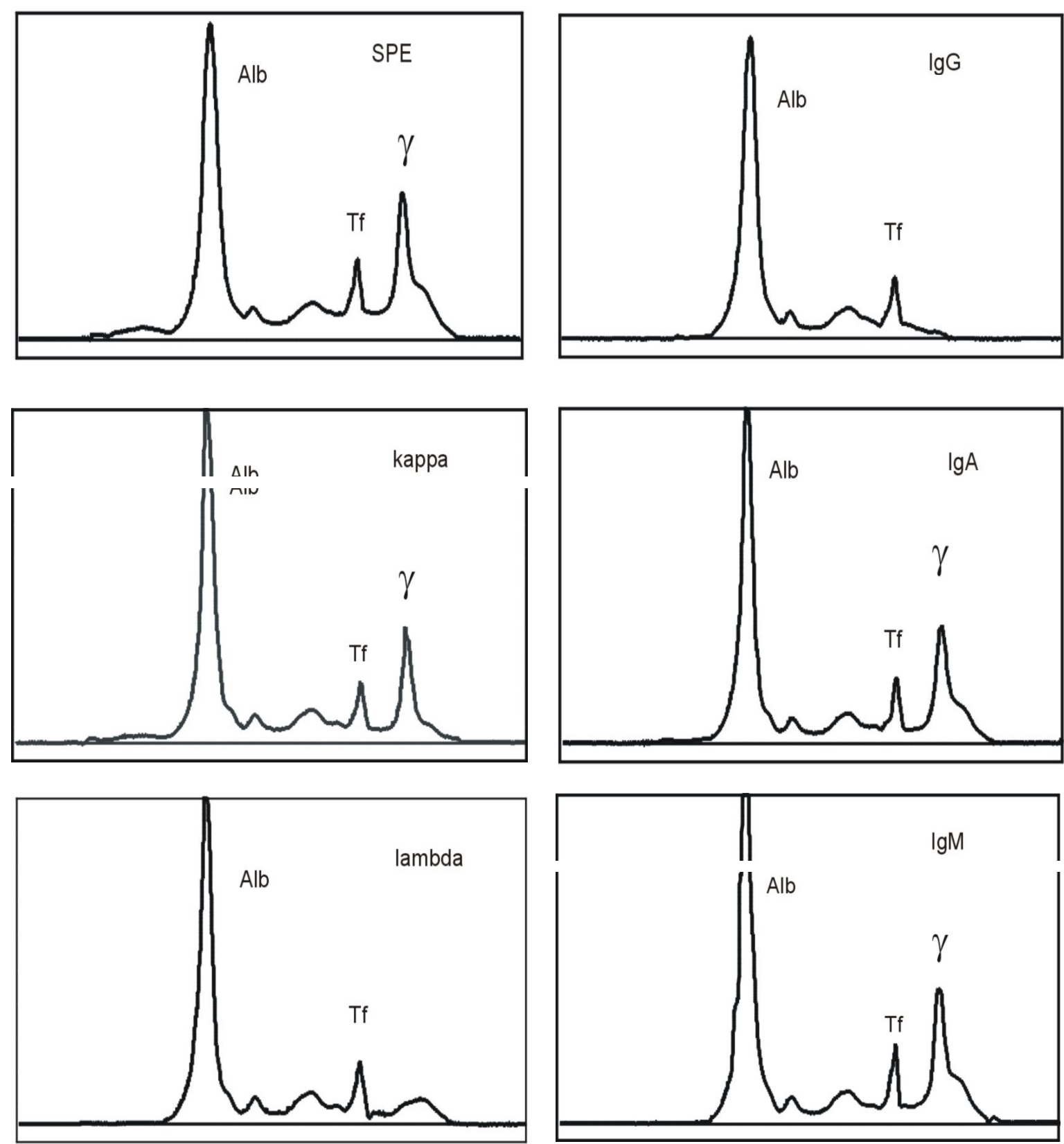

Figure 7: CZE electropherograms for the serum of Fig. 6A without immunosubtraction (SPE trace, upper left panel) and with immunosubtraction prior to analysis (type of subtraction is mentioned in upper right corner of each panel). Alb, Tf and $\gamma$ refer to albumin, transferrin and $\gamma$-region, respectively. 
with a possible asialo-Tf (Fig. 6B). In another case, a broad peak encompassing the whole region of $\gamma$-globulins from 4.9 to $5.6 \mathrm{~min}$ was noted (Fig. 6C). Serum protein electrophoresis (Fig. 7) and immunofixation (see insets in Fig. 6) was performed in all cases for further investigation of the nature and the origin of the observed peaks. In two cases, the abnormal serum pattern could be attributed to a monoclonal gammopathie of an immunoglobulin of the type IgG. Subtype specification revealed IgG lambda for both (Figs. 6A, 6B and Fig. 7). The broad double peak in the serum of Fig. 6C could be identified as a biclonal gammopathie of two distinct immunoglobulins of the type IgG lambda (see small arrows in the inset), whereas the serum whose electropherogram is depicted in Fig. 6D was found to represent a monoclonal gammopathie of an immunoglobulin of the type IgM kappa. For the four sera, CZE is predicting a normal CDT value $(0.63 \%, 0.51 \%, 0.67 \%$ and $0.52 \%$ for the data of panels $A$ to $\mathrm{D}$, respectively). Furthermore, a fair number of sera stemming from patients treated at the departmental hepatology outpatient clinic were found to contain a broad peak comigrating with the Tf isoforms whose width was larger than the entire Tf pattern and whose height is between 5 and $15 \mathrm{mAU}$. The origin of this abnormality and its influence on the CDT determination by CZE is currently under investigation and will be the subject of a forthcoming communication.

\section{Conclusions}

CZE with the dynamic CEofix double coating performed in the P/ACE MDQ permits the simultaneous, individual, quantitative determination of $\mathrm{Tf}$ isoforms in human serum and thus CDT. The precision performance assessed over a 20-day period according to the internationally accepted NCCLS EP5-A guidelines revealed the assay as being highly reproducible with within-run and total precision being dependent on the Tf isoform level and RSD values ranging between 2.2 and $14.4 \%$ (within-run precision) and 3.6 and $17.6 \%$ (total precision). Inter-day RSD values for asialo-Tf were noted to be between 9.8 and $11.5 \%$ and for disialo-Tf between 3.8 and $8.6 \%$, whereas those for CDT levels of 0.88 and $4.31 \%$ of total Tf were determined to be 8.6 and $3.4 \%$, respectively. RSD values for the isoforms with higher abundance, trisialo-Tf, tetrasialoTf, pentasialo-Tf and hexasialo-Tf, were found to be between 0.4 and $4.1 \%$. Tf patterns are recognized via detection times of $\mathrm{Tf}$ isoforms (RSD $<1.7 \%$ ) and isoform distribution, immunosubtraction and enzymatic sequential cleavage of sialic acid residues. Furthermore, heterozygous $\mathrm{Tf}-\mathrm{BC}$ and $\mathrm{Tf}-\mathrm{CD}$ variants are assigned via spiking with a known mixture of $\mathrm{Tf}$ isoforms (e.g. the serum of a healthy $\mathrm{Tf}-\mathrm{C}$ homozygote). 
Among the non-Tf peaks monitored, the CRP peak detected shortly before disialo-Tf could be identified by immunosubtraction and CRP peak magnitudes were found to correlate well with immunochemically determined CRP serum levels. The CZE assay with dynamic double coating could thereby be shown to be sensitive enough to determine elevated CRP levels in human serum. Customary serum protein CZE, immunosubtraction CZE and immunofixation analyses can be employed for the identification of unusual peaks in the $\gamma$-region. Based on our work described in this paper and previously [9], as well as that of other laboratories [16], it is now clear that CZE with a dynamic double coating can reliably be employed for the determination of CDT in human serum. This simple, rapid and highly reproducible CZE assay is an attractive alternative to HPLC and can be employed for both, screening and confirmation analysis. In analogy to HPLC, it has a high sensitivity (few false negatives) and a high specificity (few false positives) [16]. It is important to note that all data presented here were performed and evaluated according to the recommendations of the manufacturer of the CEofix CDT kit. Under these conditions, the separation of diand trisialo-Tf is typically but not always $>1.2$ [9]. Thus, baseline resolution between these two peaks was not always obtained and baseline integration with mathematical deconvolution would possibly be a more appropriate data evaluation procedure. Alternatively, modifications of the electrophoretic configurations will have to be studied. Furthermore, before adoption to the routine arena, the reference limit or cutoff value for CDT will have to be established. Both of these aspects are currently being investigated in our laboratory.

\section{Acknowledgements}

The authors acknowledge the receipt of the Analis CEofix CDT kits from its manufacturer (Analis, Namur, Belgium). This work was supported by the Liver Foundation, Bern, Switzerland and the Swiss National Science Foundation. 


\section{References}

[1] H. Stibler, K.G. Kjellin, J. Neurol. Sci. 30 (1976) 269-285.

[2] H. Stibler, C. Allgulander, S. Borg, K.G. Kjellin, Acta med. Scand. 204 (1978) 49-56.

[3] H. Stibler, S. Borg, C. Allgulander, Acta med. Scand. 206 (1979) 275-281.

[4] G. de Jong, H.G. van Eijk, Electrophoresis 9 (1988) 589-598.

[5] H.G. van Eijk, W.L. van Noort, G. de Jong, J.F. Koster, Clin. Chim. Acta 165 (1987) 141-145.

[6] T. Arndt, Clin. Chem. 47 (2001) 13-27.

[7] J.-O. Jeppsson, H. Kristensson, C. Fimiani, Clin. Chem. 39 (1993) 2115-2120.

[8] F. Tagliaro, F. Bortolotti, F. Crivellente, F. Cittadini, Forensic Sci. Rev. 12 (2000)

134-148.

[9] C. Lanz, M. Kuhn, F. Bortolotti, F. Tagliaro, W. Thormann, J. Chromatogr. A 979 (2002) 43-57.

[10] F. Crivellente, G. Fracasso, R. Valentini, G. Manetto, A.P. Riviera, F. Tagliaro, J. Chromatogr. B 739 (2000) 81-93.

[11] F. Tagliaro, F. Bortolotti, M. Zuliani, F. Crivellente, G. Manetto, V.L. Pascali, M. Marigo, J. Cap. Elec. 6 (1999) 137-143.

[12] R.P. Oda, J.P. Landers, Electrophoresis 17 (1996) 431-437.

[13] R.P. Oda, R. Prasad, R.L. Stout, D. Coffin, W.P. Patton, D.L. Kraft, J.F. O’Brien, J.P. Landers, Electrophoresis 18 (1997) 1819-1826.

[14] B. Wuyts, J.R. Delanghe, I. Kasvosve, A. Wauters, H. Neels, J. Janssens, Clin Chem. 47 (2001) 247-255.

[15] B.C. Giordano, M. Muza, A. Trout, J.P. Landers, J. Chromatogr. B 742 (2000) 79-89.

[16] F.J. Legros, V. Nuyens, E. Minet, P. Emonts, K. Zouaoui Boudjeltia, A. Courbe, J.-L. Ruelle, J. Colicis, F. de L'Escaille, J.-P. Henry, Clin. Chem. 48 (2002) 2177-2186.

[17] P.G. Righetti, C. Gelfi, B. Verzola, L. Castelletti, Electrophoresis 22 (2001) 603-611.

[18] J. Janssens, R. Chevigne, P. Louis, United States Patent No. 5,611,903, March $18,1997$.

[19] H. Katayama, Y. Ishihama, N. Asakawa, Anal. Chem. 70 (1998) 2254-2260.

[20] T.W. Graul, J.B. Schlenoff, Anal. Chem. 71 (1999) 4007-4013.

[21] L. Bendahl, S.H. Hansen, B. Gammelgaard, Electrophoresis 22 (2001) 25652573. 
[22] J.W. Kennedy, R.N. Carey, R.B. Coolen, C.C. Garber, H.T. Lee, J.B. Levine, I.M. Osberg, Evaluation of Precision Performance of Clinical Chemistry Devices; Approved Guideline. NCCLS document EP5-A, NCCLS, Wayne, PA, USA, 1999.

[23] W. Thormann, A.B. Wey, I.S. Lurie, H. Gerber, C. Byland, N. Malik, M. Hochmeister, C. Gehrig, Electrophoresis 20 (1999) 3203-3236.

[24] N.-E. Saris (Ed.), J. Clin. Chem. Clin. Biochem. 18 (1980) 69-77.

[25] M.I. Kamboh, R.E. Ferrell, Hum. Hered. 37 (1987) 65-81.

[26] R.E. Gaensslen, S.C. Bell, H.C. Lee, J. Forensic Sci. 32 (1987) 1754-1774.

[27] A. Helander, G. Eriksson, H. Stibler, J.-O. Jeppsson, Clin. Chem. 47 (2001) 1225-1233. 
B.3. CAPILLARY ZONE ELECTROPHORESIS WITH A DYNAMIC DOUBLE COATING FOR ANALYSIS OF CARBOHYDRATE-DEFICIENT TRANSFERRIN IN HUMAN SERUM: IMPACT OF RESOLUTION BETWEEN DISIALO- AND TRISIALOTRANSFERRIN ON REFERENCE LIMITS

Published in: Electrophoresis, 24 (2003) 4272 - 4281 


\title{
CAPILLARY ZONE ELECTROPHORESIS WITH A DYNAMIC DOUBLE COATING FOR ANALYSIS OF CARBOHYDRATE-DEFICIENT TRANSFERRIN IN HUMAN SERUM: IMPACT OF RESOLUTION BETWEEN DISIALO- AND TRISIALOTRANSFERRIN ON REFERENCE LIMITS
}

\author{
Christian Lanz and Wolfgang Thormann \\ Department of Clinical Pharmacology, University of Bern, \\ 3010 Bern, Switzerland.
}




\begin{abstract}
Capillary electrophoresis with a dynamic double coating formed by charged polymeric reagents represents a very effective tool for the separation of iron-saturated transferrin (Tf) isoforms and thus the determination of carbohydrate-deficient transferrin (CDT) in human serum. The resolution between di- and trisialo-Tf is dependent on the applied voltage and capillary temperature. With a $50 \mu \mathrm{m}$ I.D. capillary of about $60 \mathrm{~cm}$ total length mounted into the P/ACE MDQ, $28 \mathrm{kV}$ and $40{ }^{\circ} \mathrm{C}$, the resolution of the two $\mathrm{Tf}$ isoforms is shown to be between 1.0 and 1.4, whereas with reduced voltage and/or temperature, increased resolution at the expense of elongated run times is observed. Best data with complete resolution $\left(\mathrm{Rs} \geq 1.4\right.$ ) are obtained at $20 \mathrm{kV}$ and $30{ }^{\circ} \mathrm{C}$. For the determination of CDT in serum, incomplete separation of di- and trisialo-Tf is demonstrated to have an impact on the reference limits. Analysis of the sera of 54 healthy individuals with no or moderate alcohol consumption and using valley-to-valley peak integration, the upper (lower) reference limits for CDT in relation to total transferrin at the two power levels are $1.33(0.52)$ and $1.57(0.81) \%$, respectively, representing intervals that are significantly different $(\mathrm{P}<0.001)$. Furthermore, the reference intervals are shown to be strongly dependent on the peak integration approach used. Valley-to-valley peak integration should only be employed for conditions with complete resolution between disialo- and trisialo-Tf.
\end{abstract}




\section{Introduction}

Transferrin (Tf), the most important iron transporting protein, is a glycoprotein comprising several isoforms due to a microheterogeneity of the two carbohydrate side chains. The two N-glycans are composed of neutral sugars, forming di-, tri-, and tetraantennary structures, and zero up to eight terminal, negatively charged sialic acid residues [1]. The major $\mathrm{Tf}$ isoform, having an isoelectric point (pI) after complete saturation with iron of 5.4 and accounting for about $80 \%$ of the whole $\mathrm{Tf}$ in serum, contains four sialic acid residues and is thus referred to as tetrasialo-Tf [2]. In the 1970s Stibler et al. reported an increase of less glycosylated and therefore less sialylated Tf isoforms with $\mathrm{pI}$ values $\geq 5.7$ in the serum and cerebrospinal fluid of patients with a chronic, repeated alcohol misuse [3-5]. These isoforms encompass asialo-, monosialoand disialo-Tf and are collectively referred to as carbohydrate-deficient $\mathrm{Tf}$ (CDT). CDT is currently considered as the most specific marker for detecting a chronic, excessive alcohol intake [1]. During the past twenty years, different analytical principles for the determination of CDT have been developed, including isoelectric focusing, immunochemical determinations after anion exchange chromatography, high performance liquid chromatography (HPLC) and capillary electrophoresis (CE) $[2,6]$. $\mathrm{CE}$ in fused-silica capillaries employing dynamic capillary coatings for the prevention of proteins from adsorbing to the inner surface of the capillary turned out to be a very effective analytical tool for the separation of the major $\mathrm{Tf}$ isoforms and thus the determination of CDT [2,7-16].

Recently, two amine-based dynamic capillary coatings and a dynamic double coating that is based upon proprietary commercial reagents (CEofix-CDT kit supplied by Analis, Namur, Belgium) were compared and, for the determination of CDT, the latter approach was determined to provide the best reproducibility and highest detector responses of all three methods [15]. To produce the bilayer of the double coating, the fused-silica capillary is first rinsed with an initiator solution containing a polymeric polycation (such as Polybrene) that is adsorbed to the wall surface followed by introduction of the running buffer with a polymeric polyanion (such as poly(vinylsulfonate) that is forming a second layer and thus providing the negative charge for a strong electroosmotic flow towards the cathode. Although complete baseline resolution was not always obtained for all CDT isoforms using the CEofixCDT reagents, the outstanding precision performance of this commercial kit was shown in a comprehensive study that was based upon the NCCLS EP5-A protocol [16] and the high clinical sensitivity and specificity of this method was demonstrated by Legros et al. 
using receiver operating characteristic (ROC) plots [13,14]. The bottleneck of the CEofix-CDT test was identified as being the separation between disialo- and trisialo-Tf [15], but no systematic study about the separation of these two isoforms has been published. Furthermore, except for the estimation of the upper reference limit in the recent work of Legros et al. [14], no careful investigation of the reference limits for CDT has been reported. Thus, efforts were undertaken in our laboratory to improve the resolution of the two isoforms using the CEofix-CDT reagents and to investigate the impact of their resolution and of different peak integration schemes on the reference limits for CDT. In this paper, we wish to report our optimization strategy that includes a reduction of both the applied voltage and the temperature of the cooling liquid surrounding the capillary. Employing a reference sample group that was selected according to the guidelines of the NCCLS protocol C28-A2 and the recommendations of the international federation of clinical chemistry (IFCC) [17-23], the reference limits of CDT in human serum for analysis with the CEofix-CDT kit are established and demonstrated to be dependent on instrumental and peak integration conditions.

\section{Materials and Methods}

\subsection{Reagents, samples and sample preparation}

Buffers and reagents of the CEofix-CDT kit for the quantitative determination of CDT with the Beckman Coulter P/ACE MDQ (Kit No. 10-004740) were kindly provided by Analis (Namur, Belgium). The initiator solution comprises a $\mathrm{pH} 4.8$ malic acid/arginine buffer with the polycation and the running buffer is a $\mathrm{pH} 8.5 \mathrm{Tris} / \mathrm{boric}$ acid buffer that contains the polyanion. This kit comprises the same running buffer as that employed by Legros et al. and furnished for the Beckman Coulter P/ACE 5500 $[13,14]$. It is important to note, however, that the buffer is not identical to that employed by Wuyts et al. [10]. Patient sera were obtained from the departmental analytical laboratory where they were received for determination of CDT. Blood samples $(5 \mathrm{~mL})$ of 64 University employees were drawn by a trained nurse or a physician of the hepatology outpatient clinic. They were collected by venipuncture using native plastic tubes without additives (Monovette, white cap, Sarstedt, Sevelen, Switzerland) and centrifuged after clotting at 2'000 g for $10 \mathrm{~min}$. The supernatant was stored in $8.0 \mathrm{~mL}$ polypropylene tubes and CDT was monitored directly after centrifugation or after storage at $-20{ }^{\circ} \mathrm{C}$. Frozen samples and control sera were defrosted in a water bath at 37 ${ }^{\circ} \mathrm{C}$ for $15 \mathrm{~min}$. All samples were vortex mixed. Then, $60 \mu \mathrm{L}$ of serum or control serum and $60 \mu \mathrm{L}$ of the ferric solution of the kit (Analis) were combined and vortex mixed in a $0.5 \mathrm{~mL}$ polypropylene vial, and the vial was put into the corresponding sample tray of 
the P/ACE MDQ (Beckman Coulter, Fullerton, USA). No incubation time for the saturation of the sample with iron was required.

\subsection{Instrumentation, data evaluation and running conditions}

CZE analyses were performed on a P/ACE MDQ capillary electrophoresis system (Beckman Coulter), which was equipped with a fused-silica capillary (Polymicro Technologies, Phoenix, AZ, USA) of $50 \mu \mathrm{m}$ I.D. and $60.2 \mathrm{~cm}(50.0 \mathrm{~cm}$ to the detector $)$ total length. In contrast to the previous publications from our group, sample injection was not effected from microtiter plates $[15,16]$, but $0.5 \mathrm{~mL}$ polypropylene vials with the corresponding sample tray were used. Samples were introduced by negative pressure of $0.3 \mathrm{psi}(1 \mathrm{psi}=6894.76 \mathrm{~Pa})$ for $8.0-12.5 \mathrm{~s}$, resulting in calculated plug lengths between 0.64 and $0.82 \%$ of the effective capillary length. The temperature control of the capillary cartridge was varied between 20 and $40{ }^{\circ} \mathrm{C}$ and the temperature of the sample tray was set to $15^{\circ} \mathrm{C}$. The applied voltage was 20 or $28 \mathrm{kV}$. A $200 \mathrm{~nm}$ interference filter was used for UV detection.

Data registration and evaluation were carried out with the 32 Karat software, version 4.01 (Beckman). If not stated otherwise, peak areas were determined by valleyto-valley integration and the data were evaluated on the basis of corrected peak areas (peak area divided by detection time). The amount of single Tf isoforms and CDT (sum of asialo- and disialo-Tf) were expressed as area \% in relation to the sum of the corrected peak areas of all detected Tf isoforms. Resolution (Rs) between two peaks was calculated according to $\mathrm{Rs}=1.18\left(\mathrm{t}_{2}-\mathrm{t}_{1}\right) /\left(\mathrm{W}_{0.5(1)}+\mathrm{W}_{0.5(2)}\right)$ where $\mathrm{t}_{\mathrm{i}}$ and $\mathrm{W}_{0.5(\mathrm{i})}$ represent detection time and peak width at half height of the firstly $(i=1)$ and secondly $(i=2)$ detected peaks, respectively. An Rs value $\geq 1.40$ represents baseline separation. For calculation of the theoretical plug length, the CE Expert software, version 1.0 (Beckman) was employed.

At the beginning of each day, the capillary was rinsed with $0.2 \mathrm{M} \mathrm{NaOH}$ for 5 min with a pressure of 20 psi applied from the inlet end, followed by $0.2 \mathrm{M} \mathrm{NaOH}$ by applying simultaneously a pressure of 20 psi from the inlet to the outlet end and a constant current of $80 \mu \mathrm{A}$ for $10 \mathrm{~min}$. The same procedure was employed for the conditioning of new capillaries. At the end of a day the capillary was rinsed with $0.2 \mathrm{M}$ $\mathrm{NaOH}$ and water for 5 min each with a pressure of 60 psi applied from the inlet end. Capillaries were stored wet with the ends kept in water. Between runs the capillary was first rinsed with $0.2 \mathrm{M} \mathrm{NaOH}$ from the inlet to the outlet end with a pressure of $60 \mathrm{psi}$ for 1 min followed by $0.2 \mathrm{M} \mathrm{NaOH}$ by simultaneous application of a pressure of $20 \mathrm{psi}$ at the inlet end and a constant current of $80 \mu \mathrm{A}$ for $1.5 \mathrm{~min}$. At the beginning of a new 
run, the capillary was conditioned by applying a pressure of 15 psi from the inlet side first with the initiator buffer for $1.5 \mathrm{~min}$ and then with the run buffer of the CDT kit for 2 min.

\subsection{Study design for establishment of reference limits}

All participants had to fill out a detailed questionnaire and had to sign an informed consent confirming that they had received and understood the provided information and correctly answered to the questions. The questionnaire comprised general questions about ethnic background, gender, age, body mass, height, last blood donation, smoking habits, sport and the time point of the last meal before blood withdrawal. Other questions dealt with the health (personal feeling, time point and nature of an illness during the three preceding days, chronic or inherited diseases, increased blood fat levels and elevated blood pressure), intake of drugs, hormones and vitamins, and the following of a special diet. Women were asked about pregnancy and lactation. All volunteers had to declare their drinking habits for the last six weeks prior to blood withdrawal. Complete abstainers and people drinking on average less or more than $20 \mathrm{~g}$ pure ethanol daily could thereby be distinguished. Furthermore, all participants had to declare whether they wanted to be informed or not in the case of the detection of an abnormality in their serum pattern. Blood samples and the corresponding envelopes containing the questionnaire were blinded by the nurse. The key was given to the physician supervising the investigation. Participants that were considered to be healthy according to the information reported in the questionnaire were included. Smokers, persons taking drugs and those who reported an elevated blood pressure or increased blood fat levels were not a priori excluded. Exclusion criteria were diagnosis of chronic and systemic illnesses (disorders of the kidney, the liver, the heart, the lung, diabetes mellitus and other metabolic disorders, anemia), recent illnesses, subjective indisposition, inherited diseases, pregnancy and lactation. Volunteers reporting a drinking habit of more than $20 \mathrm{~g}$ pure ethanol per day were also excluded.

\subsection{Statistical treatment}

Statistical analyses for the establishment of the CDT reference limits were performed using the SPSS for windows student version software, version 10.0.5 (SPSS, Chicago, Illinois, USA). Input groups were compared with the Wilcoxon signed rank test or the Mann-Whitney rank sum test and each set of data was subjected to the Kolmogorov-Smirnov normality test. Boxplots were determined with SigmaStat for 
Windows version 1.0 (Jandel, Corte Madera, CA, USA) and graphical presentations were made with SigmaPlot for Windows version 4.00 (SPSS).

\section{Results and Discussion}

\subsection{Impact of instrumental conditions on transferrin isoform separation and precision}

In a previous publication it was shown that employing the CEofix-CDT kit according to the manufacturer's instructions resulted in a good but not baseline separation of di- and trisialo-Tf. For sera of healthy individuals and alcohol abusers, the resolution between these two peaks was noted to be typically $<1.3$ (Fig. 1A). Lowering the temperature with otherwise identical conditions led to a better separation between the two isoforms [15]. Thus, the effect of different combinations of temperature and voltage on run time and the resolution of di- and trisialo-Tf was investigated. To exclude an impact of the initial sample plug length on the separation, the injection time was varied between 8 and $12.5 \mathrm{~s}$ to obtain a theoretical plug length of about $0.66 \%$ of the effective capillary length in all cases (Table 1). It is important to note that the injected sample volume was somewhat smaller than that introduced using the instrumental conditions proposed by the manufacturer of the reagent kit $(10 \mathrm{~s}$ injection resulting at $40{ }^{\circ} \mathrm{C}$ in a theoretical plug length of $0.82 \%$ of the effective capillary volume). Table 1 summarizes the performance data obtained in our investigation. For each instrumental setting, three measurements with separate sample preparation were performed using the serum of an individual with a trisialo-Tf content of about $8 \%$ (Fig. 1, top graphs). Typical trisialo-Tf values were found to range between 3.5 and $6.5 \%$ $[16,24]$. Thus, the trisialo-Tf content of the chosen sample is higher than normal and does somewhat hamper the separation of disialo- and trisialo-Tf. According to Helander et al. it can be classified as a sample with a high trisialo-Tf level $(>7 \%$ of total transferrin, frequency of about $1 \%$ ) [24].

The shortest detection times and thus the shortest total time of analysis (17.0 min) was achieved with $28 \mathrm{kV}$ and a capillary cartridge temperature of $40 \mathrm{C}^{\circ}$. These conditions led to the highest power level $(1.14 \mathrm{~W} / \mathrm{m})$ and the worst separation between disialo- and trisialo-Tf, with the resolution being 1.267 (95\% confidence interval: 1.230 - 1.304). Increasing the migration time by lowering the temperature and / or the voltage provided an increase of peak resolution between di- and trisialo-Tf and resulted in an augmentation of the value of disialo-Tf (Table 1). The positive impact on peak separation by reducing the power level was less pronounced compared to that noted by 


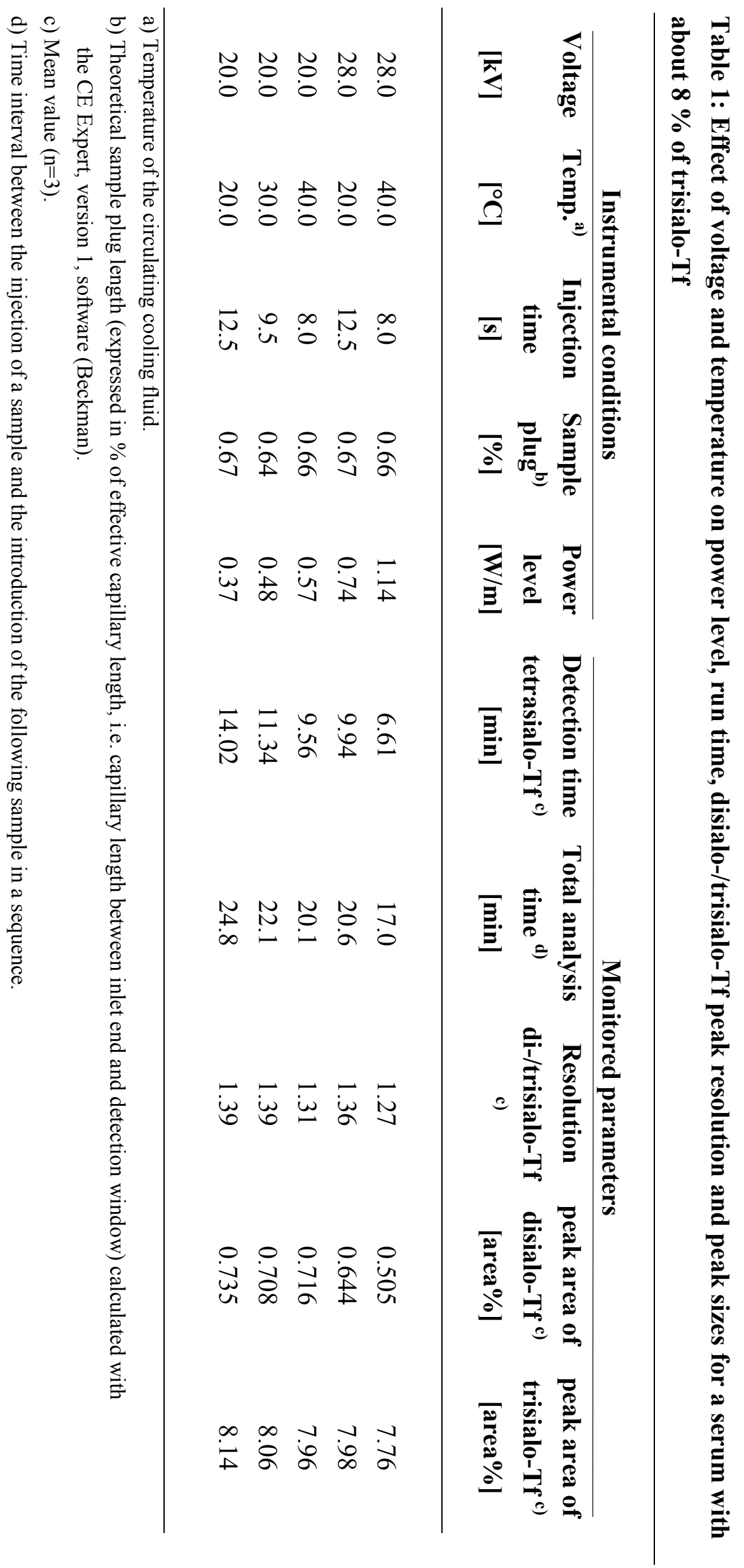


increasing the migration times, thus indicating that the quality of the separation of disialo-Tf is essentially dependent on the time the analyte is migrating in the capillary bfore reaching the detection window. Best results in terms of separation between disialo- and trisialo-Tf were obtained with $20 \mathrm{kV}$ and the temperature control of the capillary cartridge set to $30^{\circ} \mathrm{C}$ (power level of $0.48 \mathrm{~W} / \mathrm{m}$ ). The resolution between the two isoforms was determined to be 1.391 (95\% confidence interval: $1.314-1.468$ ). With this configuration baseline resolution of all $\mathrm{Tf}$ isoforms was essentially achieved within $12.1 \mathrm{~min}$ (Fig. 1B). Compared with the data obtained at $28 \mathrm{kV}$ and $40{ }^{\circ} \mathrm{C}$ (Fig. 1A), the amount of disialo-Tf detected was $40.2 \%$ higher (Table 1). Further decrease of
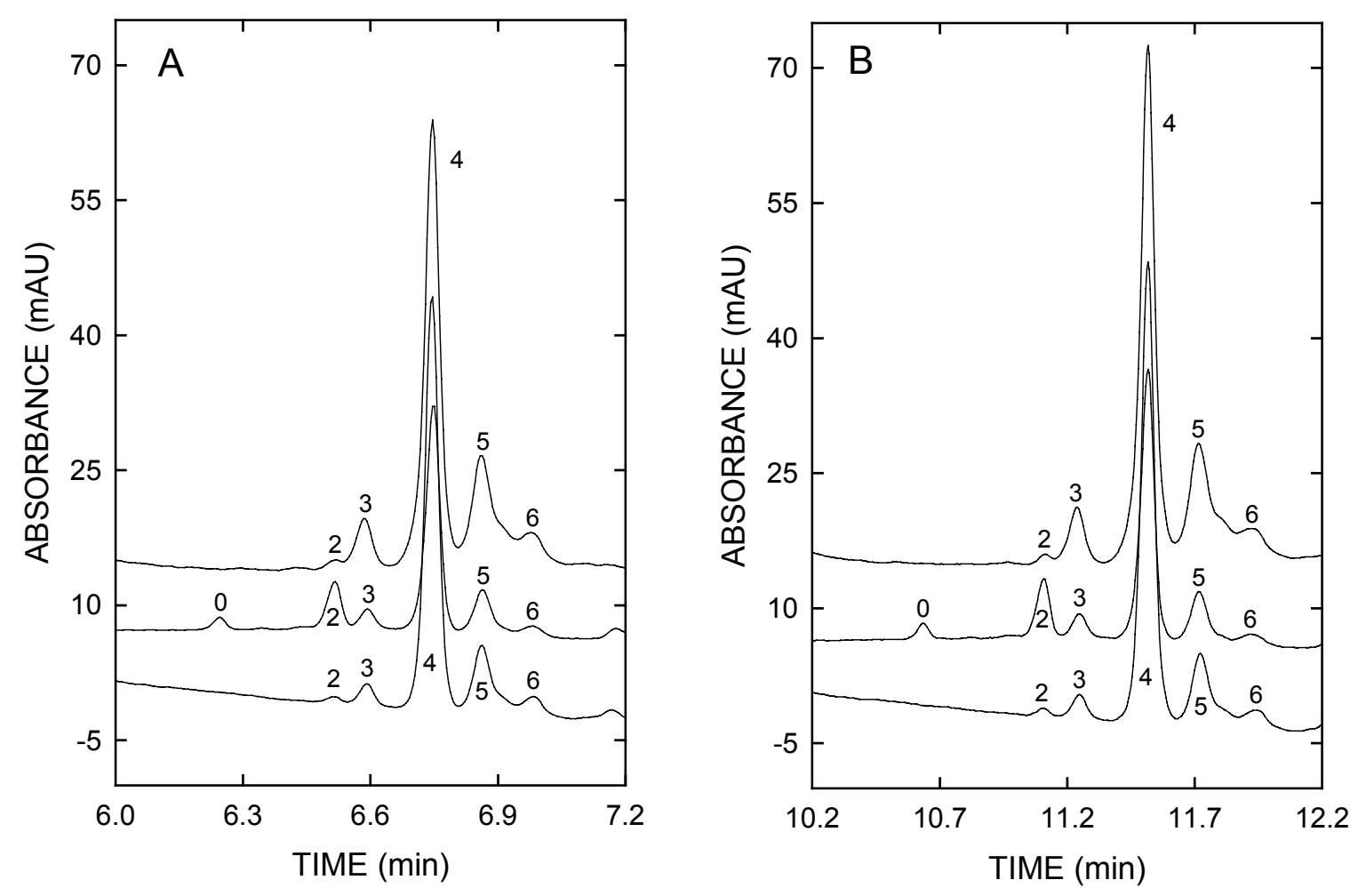

Figure 1: Typical CZE Tf patterns obtained with a sample containing a low amount of CDT (bottom graphs), a patient sample with a CDT level above the reference limit (center graphs) and a serum sample with a normal CDT value and about $8 \%$ trisialo-Tf (top graphs), respectively. CZE analysis were performed with an applied voltage of (A) 28.0 and (B) $20.0 \mathrm{kV}$, a capillary cartridge temperature of (A) 40.0 and (B) $30.0{ }^{\circ} \mathrm{C}$, and sample injection at 0.3 psi for (A) $8.0 \mathrm{~s}$ and (B) $9.5 \mathrm{~s}$. The CDT values from bottom to top were determined to be (A) $0.90 \%, 15.61 \%$ and $0.48 \%$, respectively, and (B) $1.08 \%$, $15.83 \%$ and $0.65 \%$, respectively. The electropherograms are presented with $\mathrm{y}$-axis offsets and the $\mathrm{x}$-axis of the second and third electropherograms were adjusted for equal appearance of tetrasialo-Tf. Key: 0: asialo-Tf, 2: disialo-Tf, 3: trisialo-Tf, 4: tetrasialo-Tf, 5: pentasialo-Tf, 6: hexasialo-Tf. 
the capillary temperature to $20{ }^{\circ} \mathrm{C}$ did not lead to a further improvement of the separation between disialo- and trisialo-Tf.

To evaluate the precision performance at the two power levels, customary interday and intra-day precision data were assessed with sera containing normal and elevated amounts of CDT (Table 2). For these experiments, the trisialo-Tf levels were between 4.3 and $5.6 \%$ and the sample injection time was $10 \mathrm{~s}$ as is recommended by the manufacturer of the reagent kit. For all samples analyzed, the resolution between di- and trisialo-Tf and thus the accuracy of CDT determination could thereby be shown to become significantly improved with operation at reduced voltage and temperature. Complete resolution of di- and trisialo-Tf was noted only for the lower power level which is in agreement with the data of Table 1. Furthermore, for the determination of CDT at reduced power, the RSD data are shown to become less than half compared to those obtained under the conditions recommended by the manufacturer. The modified instrumental conditions led to a highly reproducible and stable method with a baseline resolution between di- and trisialo-Tf that is required for the accurate determination of CDT. This approach with an injection time interval of $9 \mathrm{~s}$ is referred to as optimized method in the remainder of this manuscript. Accordingly, the standard method refers to the conditions at $28 \mathrm{kV}, 40{ }^{\circ} \mathrm{C}$ and an injection time of $10 \mathrm{~s}$.

\subsection{Description of the reference sample group}

Clinical chemistry data are intended to assist a diagnosis or to monitor the effectiveness of a treatment [25] and are compared with a reference interval or a reference limit derived from the statistic distribution of the reference values of a reference sample group. Based on the internationally accepted guidelines provided in the protocol NCCLS C28-A2 and the recommendations of the international federation of clinical chemistry [17-23], it was intended to elucidate health-associated reference limits for CDT. After careful evaluation of the information reported by the 64 volunteers, seven samples had to be excluded. Three volunteers reported a drinking habit with more than $20 \mathrm{~g}$ pure ethanol per day, one participant was pregnant and three persons stated to have diseases falling under the exclusion criteria. After measurement of the sera of the remaining individuals, three additional persons had to be excluded. Two samples revealed the presence of genetic Tf-B variants from Tf-BC heterozygote individuals $[10,16,24]$ and one serum contained a much higher CDT level with detectable asialo-Tf than all other samples of the reference sample group and had thus to be excluded after testing for outliers according to the NCCLS protocol [17]. Finally, the data of 54 persons could be included into the statistical calculations for the 


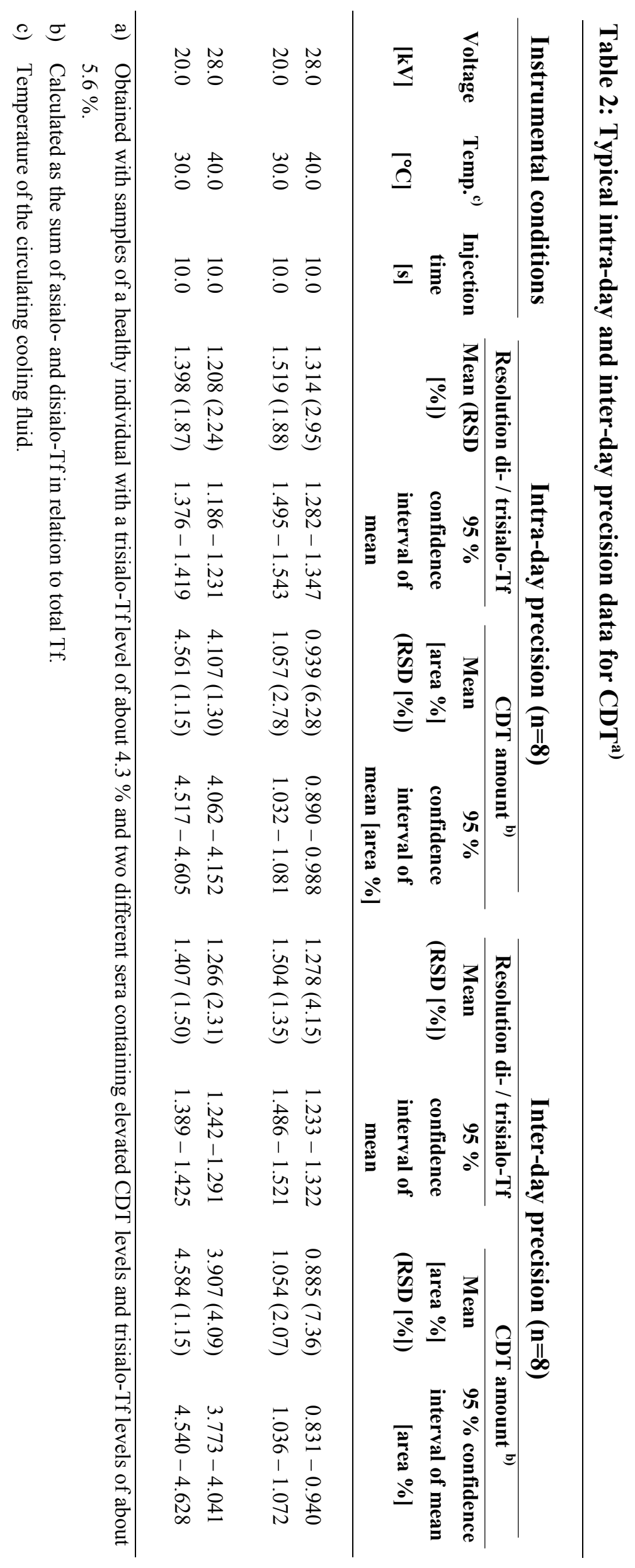


determination of the reference limits of CDT. 53 volunteers were Caucasians and one was Asian. The 18 males and 36 females had a median age of 34.5 years (range: $22-60$ years) and a median body mass index of $22.5 \mathrm{~kg} / \mathrm{m}^{2}$ (range: $17.2-26.8 \mathrm{~kg} / \mathrm{m}^{2}$ ). 6 of 54 persons reported to be smokers, and $68.5 \%$ of the volunteers (37 people) were regularly exercising with a median of $3.0 \mathrm{~h}$ per week (range: $1-20 \mathrm{~h}$ per week) and a median intensity of 5.0 (range from 2 to 10) on a scale from 1 to 10 . Blood was withdrawn in a median $2.5 \mathrm{~h}$ (mean $3.9 \mathrm{~h}$, range $0.5-17 \mathrm{~h}$ ) after the last meal or intake of beverages other than water and all participants reported to feel healthy on the day of blood withdrawal. One participant was included with elevated cholesterol, but nobody indicated having high blood pressure. 13 persons stated to be under long term treatment with drugs, with 10 women taking hormones. Other drugs declared were antihistamines, preparations of ginseng and valerian. Additionally, intake of drugs in the last three days before blood withdrawal was reported by seven volunteers. Drugs taken encompassed aspirin, ibuprofen, paracetamol, antiphlogistic eye drops, a combination of antibiotics, proton pump blockers and melatonin. Vitamins were taken by six people, and one vegetarian was participating in the study. $18.5 \%$ (10 persons) of the volunteers were abstainers and all others reported an alcohol intake of less than $20 \mathrm{~g}$ pure ethanol per day.

\subsection{Determination of the reference limits of CDT}

The reference interval is usually calculated as the central interval of reference values and is bracketed by two reference limit values at defined percentiles, thus covering a defined percentage of the values observed in the reference sample group and predicted for the reference population [17]. In the case of CDT only the upper reference limit is important for assisting the diagnosis of an elevated alcohol intake. The reference limit values were calculated by non-parametric methods from the $2.5^{\text {th }}$ and $97.5^{\text {th }}$ percentiles of the disialo-Tf reference values of the 54 reference samples (asialo-Tf was not detected in these samples) and are thus independent of the underlying distribution. It was shown above that the instrumental conditions have an influence on the value of disialo-Tf and thus CDT. It was therefore considered to be useful to measure the reference samples at the two different power levels, i.e. the original instrumental conditions proposed by the manufacturer of the kit $\left(28 \mathrm{kV}, 40{ }^{\circ} \mathrm{C}, 10 \mathrm{~s}\right.$ sample injection) and with the optimized method at reduced power $\left(20 \mathrm{kV}, 30{ }^{\circ} \mathrm{C}\right.$ and $9 \mathrm{~s}$ sample injection). The obtained disialo-Tf in relation to total $\mathrm{Tf}$ data of all 54 samples are presented in Fig. 2 and Table 3 and are referred to as \% CDT. According to the Wilcoxon signed rank test, the two groups of input data were determined to be 
statistically different $(\mathrm{P}<0.001)$ and were found to represent normal distributions $(\mathrm{P}=$ 0.159 and $\mathrm{P}=0.084$ for the conditions with higher and lower power, respectively). Linear regression analysis of the two sets of data revealed a linear relationship $(r=$ $0.904)$ with a correlation equation of $\mathrm{y}=0.95 \mathrm{x}+0.30$ where $\mathrm{x}$ and $\mathrm{y}$ refer to the CDT values obtained with the standard and optimized methods, respectively. Under $28 \mathrm{kV}$ and $40{ }^{\circ} \mathrm{C}$, the lower and upper reference limits were determined to be 0.52 and $1.33 \%$, respectively. For operation at $20 \mathrm{kV}$ and $30{ }^{\circ} \mathrm{C}$, the corresponding values were calculated to be 0.81 and $1.57 \%$, respectively. Not surprisingly, these values are similar to those obtained with the mean \pm 2 to $3 \mathrm{SD}$ (Table 3 ). It is important to add that the storage of the sera at $-20{ }^{\circ} \mathrm{C}$ and reanalysis within one month resulted in indistinguishable data $(\mathrm{P}=0.114)$. For our small set of samples and without consideration of other aspects (e.g. age and gender distributions), comparison of the values of the teetotalers with those of the moderate drinkers using the Mann-Whitney

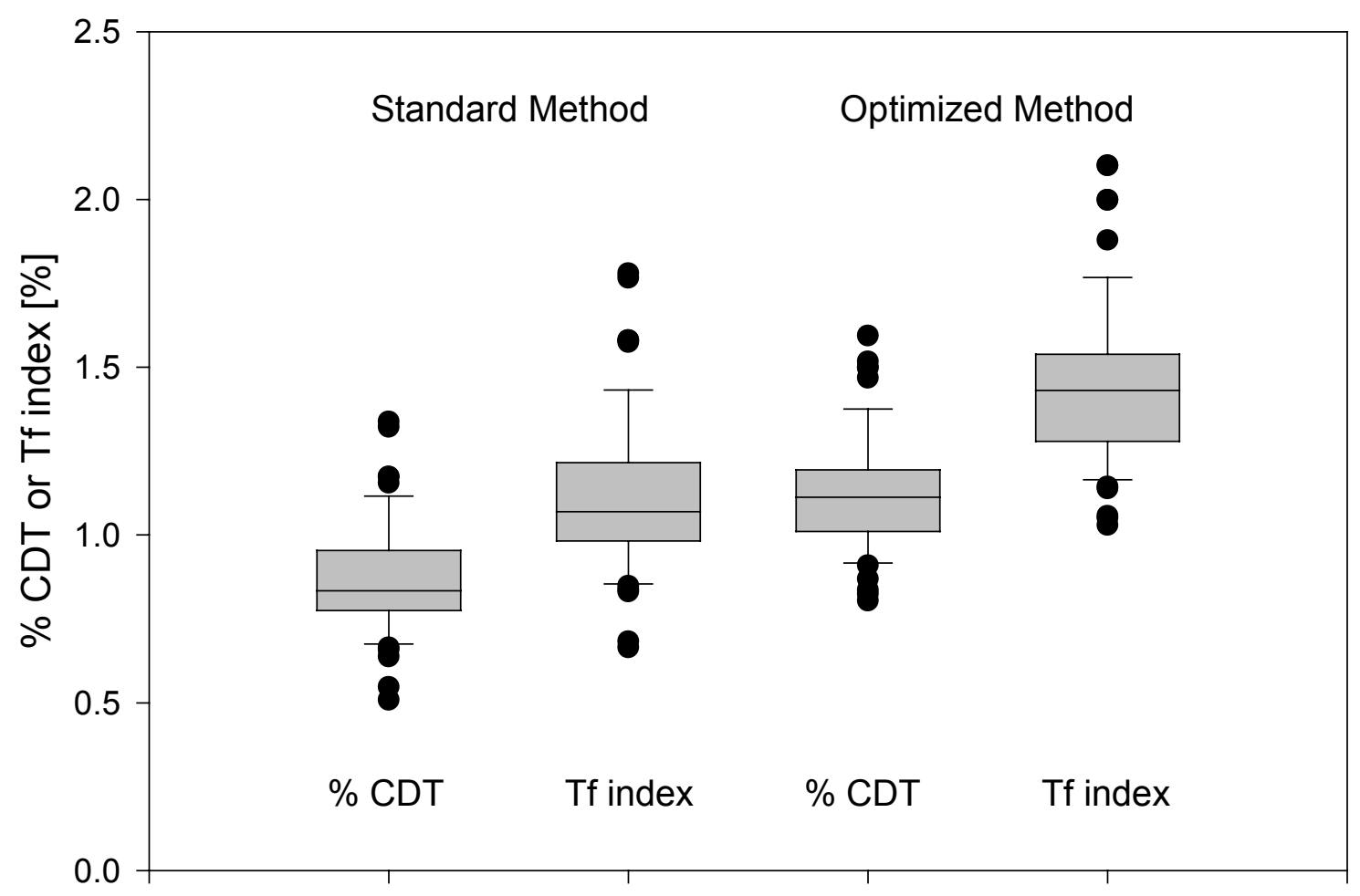

Figure 2: Box plots of the CDT data obtained for the 54 reference individuals using the standard conditions $\left(28.0 \mathrm{kV}, 40.0^{\circ} \mathrm{C}, 10 \mathrm{~s}\right.$ injection) and the optimized method $\left(20 \mathrm{kV}, 30{ }^{\circ} \mathrm{C}, 9.0 \mathrm{~s}\right)$. "\% CDT" refers to area $\%$ of disialo-Tf in relation to total $\mathrm{Tf}$ and "Tf index" refers to area \% of disialo-Tf in relation to tetrasialo-Tf. The box represents the interquartile range with the median. The whiskers extend to the $10^{\text {th }}$ and $90^{\text {th }}$ percentiles. 


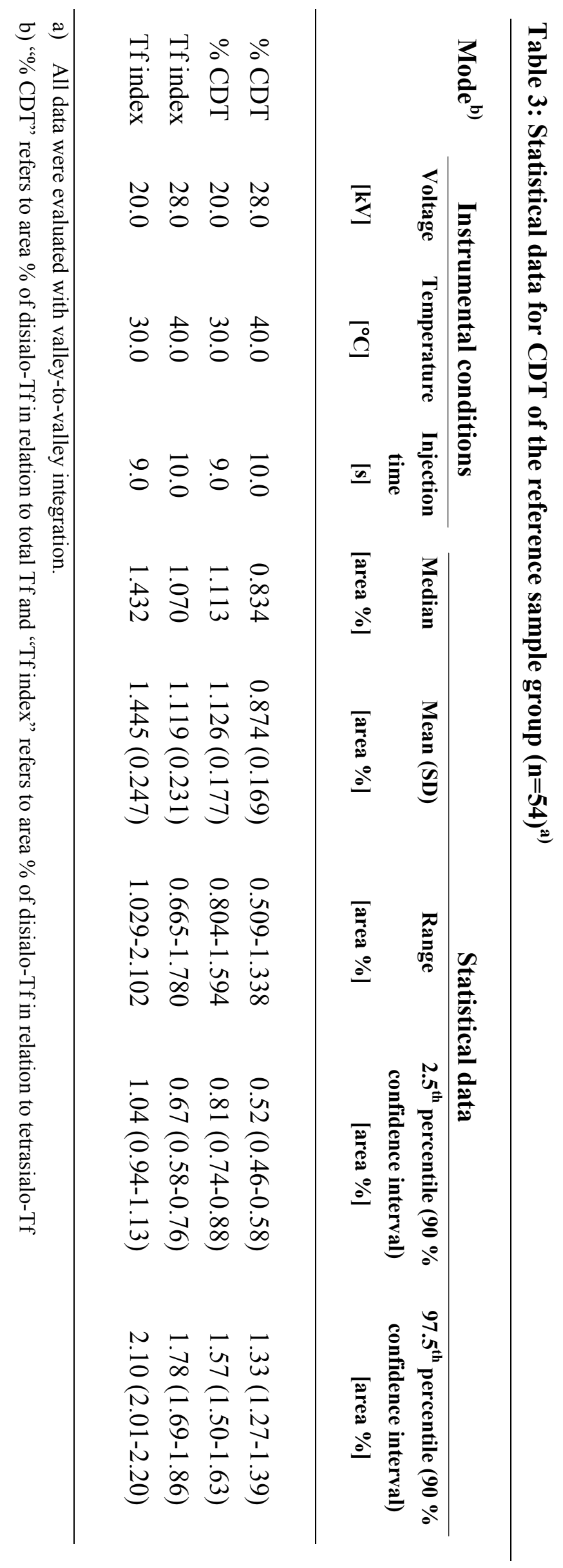


rank sum test did not provide a statistical difference $(\mathrm{P}=0.680)$. The same was found to be true for the comparison of the values of the females with those of the males $(\mathrm{P}=0.251)$. Using valley-to-valley peak integration as suggested by the manufacturer of the reagents, these data clearly indicate that the reference limits for CDT are dependent on the instrumental settings. Incomplete separation of di- and trisialo-Tf results in values that are lower compared to those when the two isoforms are completely resolved. The same was found to be true for the $\mathrm{Tf}$ index $[8,15]$, i.e. data evaluation based upon area $\%$ of disialo-Tf in relation to tetrasialo-Tf (Fig. 2, Table 3).

Based on our data produced under different instrumental conditions, the upper reference limit for \%CDT is shown to vary between 1.33 and $1.57 \%$, values that are in agreement with data produced by HPLC for which upper reference limits are reported to be between 0.8 and $1.7 \%$ [6,24,26]. Furthermore, corresponding values for the Tf index were found to be between 1.78 and $2.10 \%$ with the latter value being close to the 2.27 $\%$ reported by Tagliaro et al. [8] for a different CE-based assay. The recently reported $1.2 \%$ limit that was established on the P/ACE 5500 [14], an instrument that features the same liquid capillary cooling as the P/ACE MDQ employed in our laboratory, with the same reagents, indicates an insufficient resolution of di- and trisialo-Tf under the conditions used in that work. It is important to realize that the adoption of this reference limit could lead to misinterpretation of data. E.g. for a set of 235 patient samples analyzed in our laboratory with the method at reduced power, $27.2 \%$ of the patient samples revealed CDT values between 1.21 and $1.60 \%$. With a reference limit of 1.60 $\%$, these data are considered to be negative, whereas using the $1.2 \%$ reference limit, they would be classified as being positive. Similarly, $8.9 \%$ of the CDT values were between 1.41 and $1.60 \%$.

\subsection{Impact of peak integration on CDT value}

The data discussed thus far were all generated using valley-to-valley integration of all $\mathrm{Tf}$ isoform peaks. For elucidation of the impact of various peak integration schemes on the CDT reference limits, the data of the 54 reference samples were analyzed by four different approaches that were available with the 32 Karat software of the P/ACE MDQ, namely valley-to-valley, baseline, extended baseline and a combination of baseline (for disialo- and trisialo-Tf only) and valley-to-valley integration (Fig. 3, Table 4). A sophisticated software for accurate determination of the parameters of unresolved peaks, such as the PeakFit program of Jandel, was not 
available. The data presented in Fig. 3 were obtained with a reference sample that also contained a small peak of C-reactive protein (CRP), a peak that was detected in front of

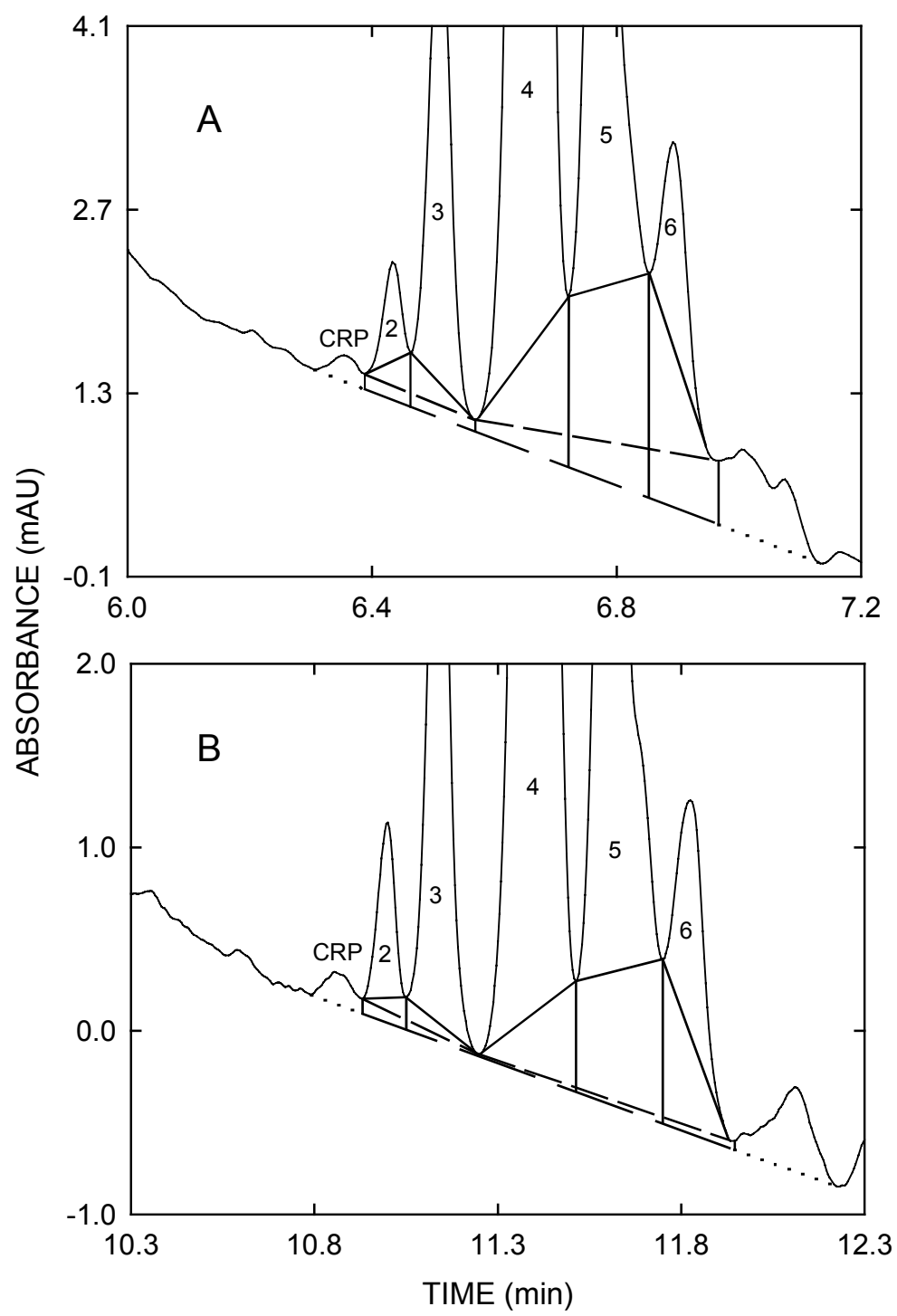

Figure 3: Visualization of the various peak integration methods for analysis of a reference sample with (A) the standard method and (B) the optimized method. The solid lines at the base of each peak refer to valley-to-valley integration. Baseline and extended baseline are marked with short dash lines and long dash lines, respectively. The dotted lines indicate the positioning of the extended baseline. For valley-tovalley, baseline, extended baseline and baseline/valley-to-valley integration, calculated Rs values for the separation between disialo- and trisialo-Tf are (A) 1.37, 1.25, 1.20 and 1.25, respectively, and (B) 1.55, 1.51, 1.48 and 1.51, respectively. Corresponding CDT levels are (A) 0.98, 1.29, 1.44 and $1.44 \%$, respectively, and (B) 1.27, 1.35, 1.51 and $1.45 \%$, respectively. Key: 2: disialo-Tf, 3: trisialo-Tf, 4: tetrasialo-Tf, 5: pentasialo-Tf, 6: hexasialo-Tf, CRP: C-reactive protein. 


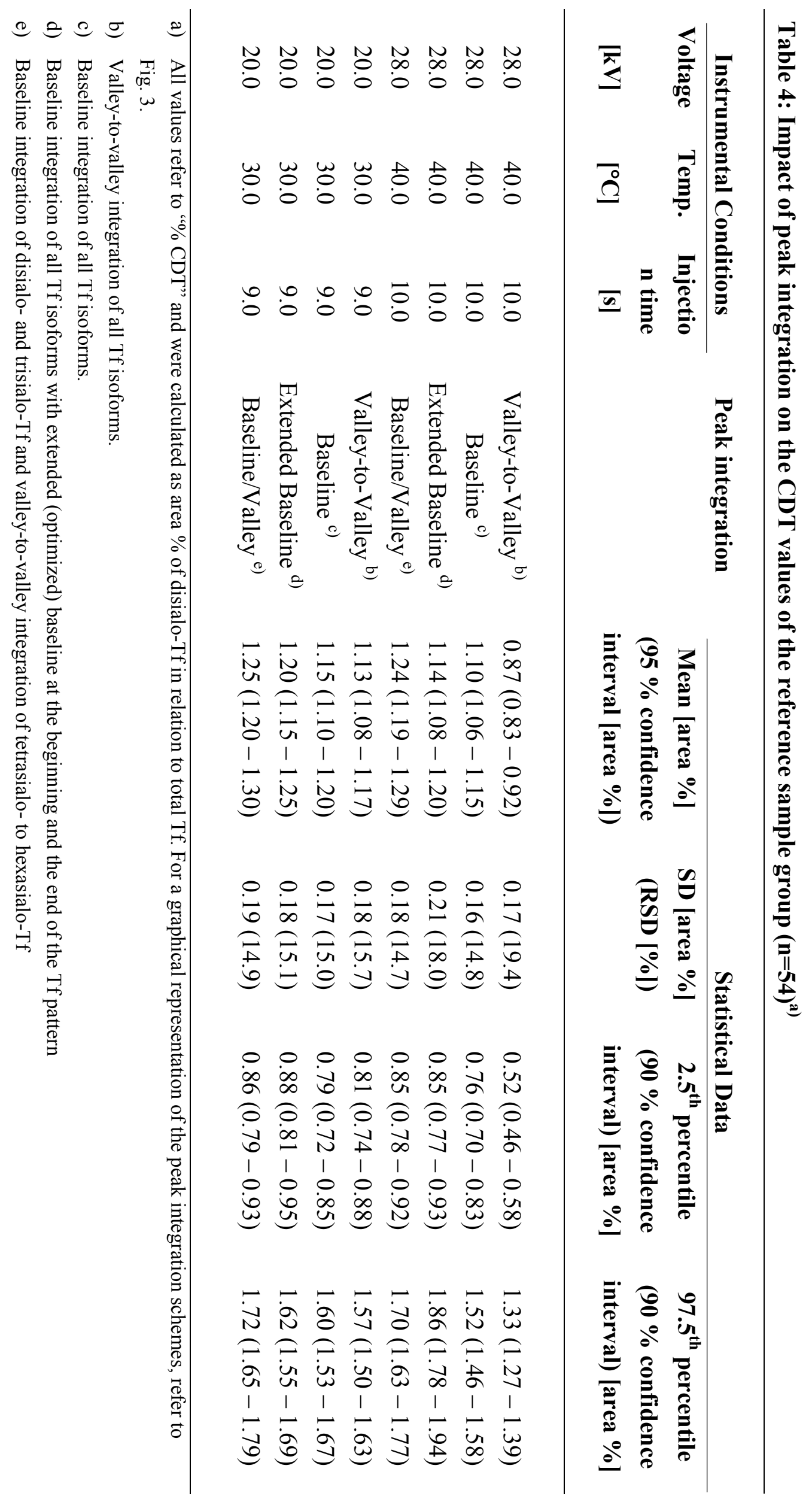


disialo-Tf and was found to correspond to a normal level $(<5 \mathrm{mg} / \mathrm{L})$ of this protein [16]. For 35 out of the 54 reference samples, CRP could not be detected at all (for an example refer to Fig. 1, bottom graphs), cases for which baseline and extended baseline under the peaks of disialo- and trisialo-Tf are almost identical. It is important to realize that the $y$ axis scales in the two panels of Fig. 3 are different and strongly elongated compared to those of Fig. 1. Not surprisingly, the separation between disialo- and trisialo-Tf was determined to be better at reduced power. Using valley-to-valley integration, Rs values for the data of Figs. $3 \mathrm{~A}$ and $3 \mathrm{~B}$ were calculated to be 1.37 and 1.55, respectively. For the different integration schemes, Rs values and CDT levels expressed as the amount of disialo-Tf in relation to total Tf are given in the legend of Fig. 3. Both, the Rs values and the CDT levels were found to be dependent on the integration scheme used.

Using valley-to-valley integration for all 54 reference samples resulted in the lowest mean, $2.5^{\text {th }}$ percentile and $97.5^{\text {th }}$ percentile values (Table 4). This was found to be true for both, the standard method $\left(28 \mathrm{kV}, 40{ }^{\circ} \mathrm{C}, 10 \mathrm{~s}\right.$ injection) and the optimized method $\left(20 \mathrm{kV}, 30{ }^{\circ} \mathrm{C}, 9 \mathrm{~s}\right.$ injection). Compared to baseline integration, the use of the extended baseline (Fig. 3) provided somewhat higher values (Table 4). For the case with incomplete separation of disialo- and trisialo-Tf (standard method), the upper reference limits for the four integration schemes were determined to range between 1.33 and 1.86 $\%$ (increase of $39.85 \%$, Table 4). A smaller difference was noted for the optimized method (1.57 to $1.72 \%$, increase of $9.55 \%)$. The data were also evaluated from a statistical point of view via pair-wise comparison of two distributions using the Wilcoxon signed rank test (Fig. 4). For the standard method (data of upper right corner of Fig. 4), all but one comparison revealed that the two groups of input data are statistically different $(\mathrm{P}<0.001)$. The data obtained with baseline and extended baseline integration were not found to be different $(\mathrm{P}=0.722$, Fig. 4). For the optimized method (data of lower left corner of Fig. 4), all compared pairs of input data were found to be statistically different $(\mathrm{P}<0.05)$. Comparison of the corresponding data of the two methods revealed differences except for the case with baseline integration for disialoand trisialo-Tf and valley-to-valley integration for all other isoforms (gray squares in Fig. 4). Furthermore, our data indicate that baseline integration for the standard method and valley-to-valley integration for the optimized method are providing almost identical reference limits ( $\mathrm{P}=0.142$, not included in Fig. 4).

Finally, employing baseline integration for disialo- and trisialo-Tf and valley-tovalley integration for all other $\mathrm{Tf}$ isoforms was found to provide the same reference limits for both methods ( $\mathrm{P}=0.304$, Fig. 4, Table 4). Using this peak integration scheme, the data obtained indicate that a common upper CDT reference limit of $1.7 \%$ could be 
employed. Furthermore, the same was found to be true for data evaluation based upon the $\mathrm{Tf}$ index. The reference intervals for the standard and optimized methods were calculated to be $1.11-2.28 \%$ and $1.11-2.29 \%$, respectively $(\mathrm{P}=0.555)$. Thus, a common upper reference limit of $2.3 \%$ could be employed for the Tf index. This value is identical to the $2.27 \%$ reported by Tagliaro et al. [8] for a different CE-based assay.

\section{STANDARD METHOD}

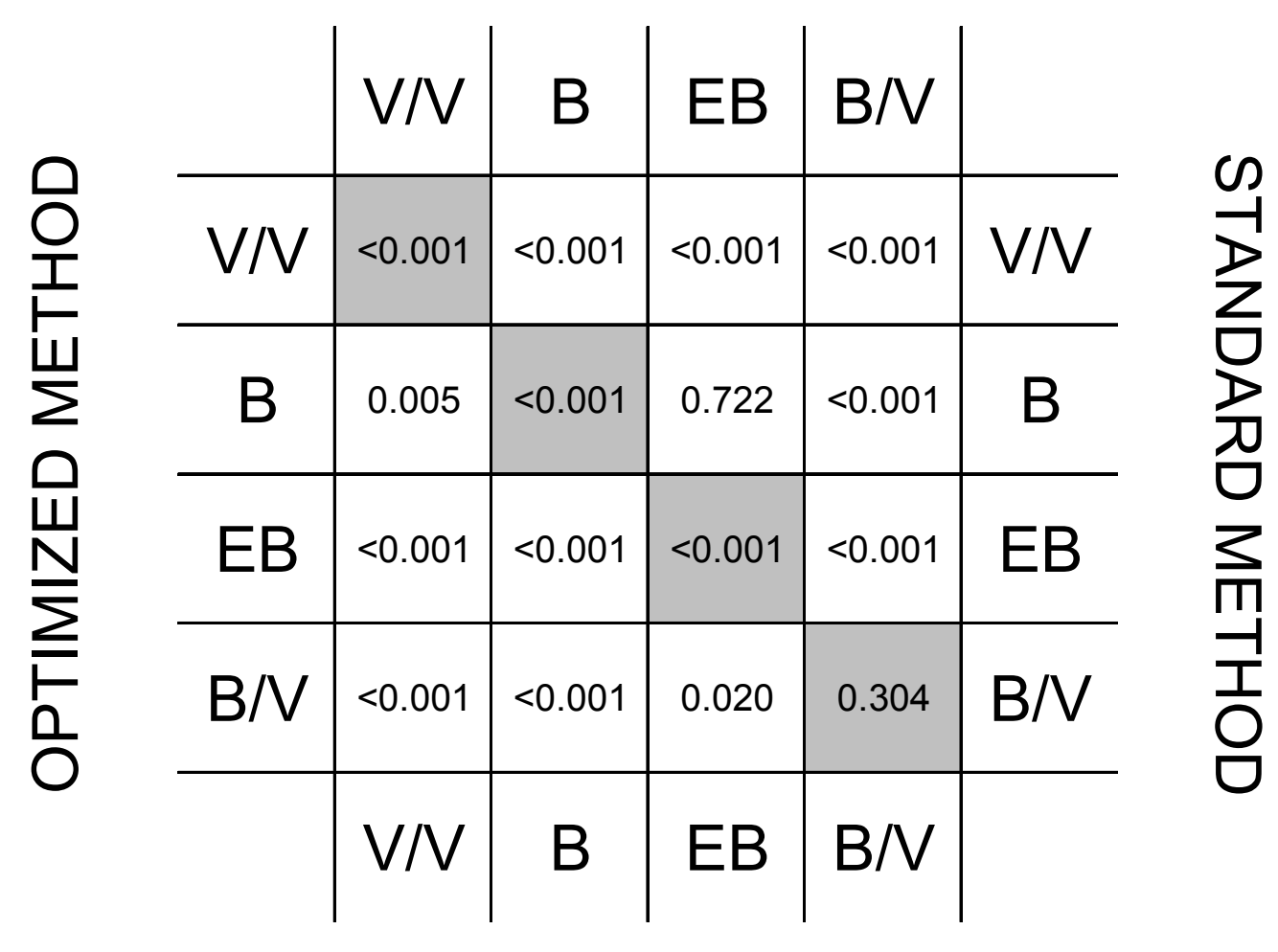

OPTIMIZED METHOD

Figure 4: Statistical data (P values) for data comparison of the 54 reference CDT values obtained with the four peak integration schemes (Table 4), namely valley-to-valley integration (V/V), baseline integration (B), extended baseline integration (EB) and baseline integration for disialo- and trisialo-Tf and valley-tovalley integration for the other $\mathrm{Tf}$ isoforms $(\mathrm{B} / \mathrm{V})$. For the standard and optimized methods, the data are centered around the upper right and lower left corners, respectively, of the overall square. The values in the gray squares refer to the comparison between the two methods. 


\section{Concluding remarks}

An important prerequisite for the accurate determination of $\mathrm{Tf}$ isoforms in serum is the absence of interfering compounds. Using immunosubtraction of $\mathrm{Tf}$ [13-16], comigrating peaks could not be detected. Very high levels of C-reactive proteins and certain immunoglobulins, however, were previously found to potentially interfere with the determination of disialo-Tf and asialo-Tf, respectively [16]. For the reference data presented in this paper, however, all samples were found to be free of these interferences. Employing the Analis CEofix-CDT kit on the P/ACE MDQ with the recommended $50 \mu \mathrm{m}$ I.D. fused-silica capillary of $60.2 \mathrm{~cm}(50.0 \mathrm{~cm}$ to the detector $)$ total length, the resolution between di- and trisialo-Tf was found to be dependent on the applied voltage and temperature. With the conditions suggested by the manufacturer of the kit $\left(28 \mathrm{kV}\right.$ and $40{ }^{\circ} \mathrm{C}, 10 \mathrm{~s}$ sample injection, power level of $\left.1.14 \mathrm{~W} / \mathrm{m}\right)$, the resolution was determined to be between 1.0 and 1.4, whereas with reduced voltage and/or temperature, increased resolution at the expense of elongated run times was observed. Best data with Rs $\geq 1.4$ were obtained at $20 \mathrm{kV}$ and $30{ }^{\circ} \mathrm{C}$ (power level of $0.48 \mathrm{~W} / \mathrm{m}$ ) and with an injection time interval between 9.0 and $10.0 \mathrm{~s}$. Furthermore, intra-day and inter-day RSD values for the determination of CDT were found to be significantly lower at the reduced power level.

For the determination of CDT in serum, incomplete separation of di- and trisialo-Tf is demonstrated to have an impact on the reference limits. Analyzing the sera of 54, carefully selected reference individuals (10 abstainers and 44 persons with moderate alcohol consumption of $<20 \mathrm{~g}$ of ethanol per day who did not reveal any asialo-Tf in their sera) with valley-to-valley peak integration and expression of disialo$\mathrm{Tf}$ in relation to total $\mathrm{Tf}$, the upper reference limits for the two power levels were determined to be $1.33 \%$ and $1.57 \%$, respectively. The corresponding lower reference limits were 0.52 and $0.81 \%$, respectively. From a statistical point of view, the two sets of data are significantly different $(\mathrm{P}<0.001)$. Similarly, such a difference was also found for the amount of disialo-Tf in relation to tetrasialo-Tf, the so-called Tf index, for which the upper reference limits were determined to be 1.78 and $2.10 \%$, respectively ( $P$ $<0.001)$. Furthermore, the data obtained indicate that baseline integration for the standard method and valley-to-valley integration for the optimized method are providing almost identical reference limits. Using baseline integration for disialo- and trisialo-Tf and valley-to-valley integration for all other $\mathrm{Tf}$ isoforms was found to provide the same reference limits for both methods $(1.7 \%$ and $2.3 \%$ for CDT data in relation to total $\mathrm{Tf}$ and tetrasialo-Tf, respectively). 
Peak integration is shown to have a considerable impact on the reference limits. For the case of incomplete separation between disialo- and trisialo-Tf (such as the standard method mentioned above) and without employing a sophisticated peak analyzing software, baseline integration is demonstrated to provide better data than valley-to-valley integration. The latter, simpler approach can however be recommended for the conditions with complete resolution between di- and trisialo-Tf, such as the optimized method described in this paper. It is demonstrated in this paper that optimization can be reached by reduction of applied electric power and capillary temperature. Alternatively, improved resolution between di- and trisialo-Tf can also be obtained via elongation of the capillary [15] or, to a lesser degree, via an adjustment of the initial sample plug length. Furthermore, as the temperature was found to play a key role for the separation of the two isoforms in question and the intracapillary temperature is strongly dependent on the applied power level and the effectiveness of the heat removal [27], a method transfer from an instrument featuring liquid capillary cooling to one with forced air thermostating will require a careful selection of the major parameters such as voltage, temperature and initial sample plug length. The same is true in case of a change of the capillary dimensions and/or material. Thus, in order to avoid that each laboratory has to elaborate on data evaluation and establishment of reference limits, CDT by CE should be determined using baseline integration of the disialo- and trisialo-Tf peaks. Valley-to-valley peak integration should only be employed for conditions with complete resolution between disialo- and trisialo-Tf.

\section{Acknowledgments}

The authors acknowledge stimulating discussions with Dr. F. Tagliaro and the receipt of the Analis CEofix CDT kits from its manufacturer (Analis, Namur, Belgium). This work was supported by the Liver Foundation, Bern, Switzerland and the Swiss National Science Foundation. 


\section{References}

[1] Arndt, T., Clin. Chem. 2001, 47, 13-27.

[2] Tagliaro, F., Bortolotti, F., Crivellente, F., Cittadini, F., Forensic Sci. Rev. 2000, $12,134-148$.

[3] Stibler, H., Kjellin, K.G., J. Neurol. Sci. 1976, 30, 269-285.

[4] Stibler, H., Allgulander, C., Borg, S., Kjellin, K.G., Acta med. Scand. 1978, 204, 49-56.

[5] Stibler, H., Borg, S., Allgulander, C., Acta med. Scand. 1979, 206, 275-281.

[6] Jeppsson, J.O., Kristensson, H., Fimiani, C., Clin. Chem. 1993, 39, 2115-2120.

[7] Crivellente, F., Fracasso, G., Valentini, R., Manetto, G., Riviera, A.P., Tagliaro, F., J. Chromatogr. B 2000, 739, 81-93.

[8] Tagliaro, F., Bortolotti, F., Zuliani, M., Crivellente, F., Manetto, G., Pascali, V.L., Marigo, M., J. Cap. Elec. 1999, 6, 137-143.

[9] Oda, R.P., Landers, J.P., Electrophoresis 1996, 17, 431-437.

[10] Wuyts, B., Delanghe, J.R., Kasvosve, I., Wauters, A., Neels, H., Janssens, J., Clin. Chem. 2001, 47, 247-255.

[11] Giordano, B.C., Muza, M., Trout, A., Landers, J.P., J. Chromatogr. B 2000, 742, 79-89.

[12] Beisler, A.T., Kelly, R.H., Landers, J.P., Anal. Biochem. 2000, 285, 143-150.

[13] Legros, F.J., Nuyens, V., Minet, E., Emonts, P., Zouaoui Boudjeltia, K., Courbe, A., Ruelle, J.-L., Colicis, J., de L'Escaille, F., Henry, J.-P., Clin. Chem. 2002, $48,2177-2186$.

[14] Legros, F.J., Nuyens, V., Baudoux, M., Zouaoui Boudjeltia, K., Ruelle, J.-L., Colicis, J., Cantraine, F., Henry, J.-P., Clin. Chem. 2003, 49, 440-449.

[15] Lanz, C., Kuhn, M., Bortolotti, F., Tagliaro, F., Thormann, W., J. Chromatogr. A 2002, 979, 43-57.

[16] Lanz, C., Marti, U., Thormann, W., J. Chromatogr. A 2003, 1013, 131-147.

[17] How to define and determine reference intervals in the clinical laboratory; approved guideline-second edition. NCCLS document C28-A2, NCCLS, Wayne, PA, USA

[18] Solberg, H.E., J. Clin. Chem. Clin. Biochem. 1987, 25, 337-342.

[19] PetitClerc, C., Solberg, H.E., J. Clin. Chem. Clin. Biochem. 1987, 25, 639-644.

[20] Solberg, H.E., PetitClerc, C., J. Clin. Chem. Clin. Biochem. 1988, 26, 593-598.

[21] Solberg, H.E., Stamm, D., Eur. J. Clin. Chem. Clin. Biochem. 1991, 29, 531 535. 
[22] Solberg, H.E., J. Clin. Chem. Clin. Biochem. 1987, 25, 645-656.

[23] Dybkær, R., Solberg, H.E., J. Clin. Chem. Clin. Biochem. 1987, 25, 657-662.

[24] Helander, A., Eriksson, G., Stibler, H., Jeppsson, J.-O., Clin. Chem. 2001, 47, 1225-1233.

[25] Approved recommendation (1978) on quality control in clinical chemistry. Part 1. General principles and terminology. J. Clin. Chem. Clin. Biochem. 1980, 18, 69-77.

[26] Turpeinen, U., Methuen, T., Alfthan, H., Laitinen, K., Salaspuro, M., Stenman, U.-H., Clin. Chem. 2001, 47, 1782-1787.

[27] Lacey, M.E., Webb, A.G., Sweedler, J.V., Anal. Chem. 2000, 72, 4991-4998. 


\section{B.4. IMPROVED CAPILLARY ELECTROPHORESIS METHOD FOR THE DETERMINATION OF CARBOHYDRATE-DEFICIENT TRANSFERRIN IN PATIENT SERA}

Published in: Electrophoresis, 25 (2004) 2309-2318 


\title{
IMPROVED CAPILLARY ELECTROPHORESIS METHOD FOR THE DETERMINATION OF CARBOHYDRATE-DEFICIENT TRANSFERRIN IN PATIENT SERA
}

\author{
Christian Lanz ${ }^{1}$, Martina Kuhn ${ }^{1}$, Veronika Deiss ${ }^{2}$ and Wolfgang Thormann ${ }^{1}$ \\ 1) Department of Clinical Pharmacology, University of Bern, \\ 3010 Bern, Switzerland.
}

2) Meditest Bulle SA, Bulle, Switzerland. 


\begin{abstract}
Capillary electrophoresis with a dynamic double coating formed by charged polymeric reagents represents a very effective tool for the separation of iron-saturated transferrin (Tf) isoforms and thus the determination of carbohydrate-deficient transferrin (CDT, sum of asialo-, monosialo- and disialo-Tf in relation to total Tf) in human serum. Using the CEofix-CDT reagents, a $50 \mu \mathrm{m}$ I.D. capillary of $60 \mathrm{~cm}$ total length and the $\mathrm{P} / \mathrm{ACE}$ MDQ under optimized instrumental conditions $\left(20 \mathrm{kV}\right.$ and $\left.30{ }^{\circ} \mathrm{C}\right)$ is demonstrated to provide outstanding assay precision for the determination of CDT in human serum. For CDT levels of $1.0 \%$ and $4.5 \%$, precision RSD values $(n=8)$ were determined to be $<3.0 \%$ and $<1.5 \%$, respectively. During the first year of operation under routine conditions, more than 600 patient samples were analyzed in a total of 62 sets of runs. Except for selected samples of patients with severe liver diseases, interference-free Tf patterns were detected. Asialo-Tf was not detected in control sera and in patient sera with a CDT level $<1.70 \%$, but became detectable in $89.6 \%$ of sera with $>2.3 \%$ disialo-Tf. Monosialo-Tf was only detected in two sera containing $>13.3$ $\%$ CDT. The optimized CZE assay was applied to confirm positive CDT results produced by an immunoassay during long-term monitoring of a patient which led to the determination of the elimination kinetics of asialo-Tf, disialo-Tf and CDT after an episode of high alcohol consumption (estimated apparent half lifes of 4.86, 7.24 and 6.74 days, respectively). The optimized CZE assay with an upper reference limit for CDT of $1.70 \%$ represents a very attractive alternative to HPLC. It features simpler sample preparation, faster analysis time and higher isoform resolution compared to the most recent HPLC approach and can thus be regarded as a new candidate of a reference method for CDT.
\end{abstract}




\section{Introduction}

The abuse of alcohol represents one of the major public health problems in many different cultures with tremendous consequences for society, medicine and economy. Selfreporting of alcohol consumption is notoriously unreliable and the diagnosis of alcohol abuse based on clinical symptoms is difficult. Therefore, laboratory markers are strongly required as diagnostic tools for an early and more reliable detection of excessive alcohol consumption and alcoholism. A big variety of compounds have been investigated and some of them like gamma-glutamyl transferase, aspartate aminotransferase, alanine aminotransferase and mean corpuscular volume are widely used in the clinical practice, however with questionable sensitivities and / or specificities [1-4].

Since its first description in the 1970 s by Stibler et. al. [5-7], carbohydratedeficient transferrin (CDT) has gained more and more attention and importance in the detection of chronic, heavy alcohol consumption and in the monitoring of alcohol abusers, including the detection of relapse drinking. CDT encompasses isoforms of the glycoprotein transferrin ( $\mathrm{Tf}$ ) with zero up to two sialic acid residues in the carbohydrate side chains of the molecule. Tf, the most important iron transporting protein, contains two carbohydrate chains with a total of up to eight end-standing sialic acid residues, with the major isoform - accounting for about $80 \%$ of total $\mathrm{Tf}$ - being tetrasialo-Tf with four sialic acid residues [2,8]. Today, CDT is considered as the most specific biological marker for the identification of chronic excessive alcohol intake [8-9].

In the last two decades, different analytical approaches for the quantitative determination of CDT in human serum have been developed, including isoelectric focusing, anion exchange chromatography followed by immunochemical determinations, high performance liquid chromatography (HPLC) and capillary zone electrophoresis (CZE) [1,2,4,10-12]. With the adoption of dynamic capillary coatings, preventing proteins from adsorbing to the capillary surface, CZE in fused-silica capillaries turned out to be a very effective analytical approach for the separation and quantitative determination of the $\mathrm{Tf}$ isoforms and thus CDT [2, 13-16]. Recently, commercial reagents producing a dynamic double coating and serving as the separation buffer (CEofix-CDT kit, Analis, Namur, Belgium) have been introduced and shown to provide the best performance characteristics in terms of reproducibility, detector response and analysis time [16]. To produce the bilayer of the double coating, the fusedsilica capillary is first rinsed with an initiator solution containing a polymeric polycation 
(such as Polybrene) that is adsorbed to the wall surface followed by introduction of the running buffer with a polymeric polyanion (such as poly(vinylsulfonate) that is forming a second layer and thus providing the negative charge for a strong electroosmotic flow towards the cathode. Further investigations with this CDT assay revealed outstanding precision performance according to the internationally accepted National Committee for Clinical Laboratory Standards (NCCLS) EP5-A protocol [17] as well as high clinical sensitivity and specificity that were determined by receiver operating characteristic (ROC) plots $[18,19]$. The bottleneck of the CEofix-CDT test was identified as being the incomplete separation between di- and trisialo-Tf [16,17], a problem that was recently addressed and optimized via reduction of applied voltage and temperature. In the same work, different peak integration schemes were evaluated and the health associated reference limits of CDT were determined under different instrumental conditions [20].

In this paper, we wish to report the assessment of the precision performance of the optimized CEofix-CDT-based method for the quantification of CDT and to provide insight into the first experience with this method under routine conditions for screening and confirmation analysis of CDT in patient sera. A discussion on the occurrence of asialo- and monosialo-Tf and the long-term monitoring of a patient revealing an episode of relapse drinking are also presented.

\section{Materials and Methods}

\subsection{Reagents and samples}

Buffers and reagents of the CEofix-CDT kit for the quantification of CDT in human serum with the P/ACE MDQ (Kit No. 10-004740) were obtained from Analis (Namur, Belgium). Rabbit anti-human Tf antibody (titer: $2800 \mathrm{mg} / \mathrm{L}$ ) was from Dako (Glostrup, Denmark) and $0.9 \%$ sodium chloride solution from Bichsel (Interlaken, Switzerland). Patient sera were from the departmental analytical laboratory where they were received for determination of CDT. They were stored in $8.0 \mathrm{~mL}$ polypropylene tubes at $-20{ }^{\circ} \mathrm{C}$. Control sera were divided into $60 \mu \mathrm{L}$ aliquots and stored in $0.5 \mathrm{~mL}$ polypropylene vials at $-20^{\circ} \mathrm{C}$.

\subsection{Sample preparation}

Frozen samples were defrosted in a water bath at $37{ }^{\circ} \mathrm{C}$ for $15 \mathrm{~min}$ and vortex mixed for a few seconds. Then, $60 \mu \mathrm{L}$ of sample and $60 \mu \mathrm{L}$ of the ferric solution of the 
reagent kit (Analis) were combined in a $0.5 \mathrm{~mL}$ polypropylene vial and vortex mixed. No further incubation time for iron saturation of the sample was required, and the vial was put into the corresponding sample tray of the P/ACE MDQ (Beckman Coulter, Fullerton, USA). For immunosubtraction of Tf, $80 \mu \mathrm{L}$ of serum was incubated with 160 $\mu \mathrm{L}$ of anti-human $\mathrm{Tf}$ antibody in a polypropylene vial for $45 \mathrm{~min}$ at room temperature. After centrifugation at $8^{\prime} 000 \mathrm{~g}$ and $4{ }^{\circ} \mathrm{C}$ for $20 \mathrm{~min}, 60 \mu \mathrm{L}$ of the supernatant was collected and combined with $30 \mu \mathrm{L}$ of the ferric solution. For data comparison with untreated sample, $30 \mu \mathrm{L}$ of serum was combined with $60 \mu \mathrm{L}$ of $0.9 \% \mathrm{NaCl}$ solution and $45 \mu \mathrm{L}$ of the ferric solution.

\subsection{Instrumentation, data evaluation and running conditions}

A P/ACE MDQ capillary electrophoresis system (Beckman Coulter), equipped with a fused-silica capillary (Polymicro Technologies, Phoenix, AZ, USA) of $50 \mu \mathrm{m}$ I.D. (375 $\mu \mathrm{m}$ O.D.) and $60.2 \mathrm{~cm}$ (50 $\mathrm{cm}$ to the detector) total length, was used. Samples were introduced from $0.5 \mathrm{~mL}$ polypropylene vials by applying a negative pressure of 0.3 psi $(1 \mathrm{psi}=6894.76 \mathrm{~Pa})$ for $9-10 \mathrm{~s}$. The temperature control of the circulating cooling fluid of the capillary cartridge was set to $30.0{ }^{\circ} \mathrm{C}$ and the temperature of the sample tray was kept at $15.0^{\circ} \mathrm{C}$. The applied voltage was $20.0 \mathrm{kV}$, resulting in a current of about $14.4 \mu \mathrm{A}$ and a power level of $0.48 \mathrm{~W} / \mathrm{m}$. It is important to note that these instrumental conditions are different from those proposed by the manufacturer of the CEofix-CDT kit that were employed in a previous publication [17] and are those found to provide optimized resolution between disialo- and trisialo-Tf without changing capillary dimensions and running buffer [20]. UV detection was effected with a $200 \mathrm{~nm}$ interference filter. The 32 Karat software, version 4.01 (Beckman) was used for data registration and evaluation. Peak areas were determined by valley-to-valley integration and the data were evaluated on the basis of corrected peak areas (peak area divided by detection time). The amounts of single Tf isoforms and CDT (sum of asialo-, monosialo- and disialo-Tf) were calculated as area \% in relation to the sum of the corrected peak areas of all detected Tf isoforms.

New capillaries were rinsed for 5 min with $0.2 \mathrm{M} \mathrm{NaOH}$ with a pressure of 20 psi from the inlet end to the outlet end, followed by $0.2 \mathrm{M} \mathrm{NaOH}$ by simultaneous application of a pressure of 20 psi from the inlet end and a constant current of $80 \mu \mathrm{A}$ for $10 \mathrm{~min}$. The same procedure was employed for the conditioning at the beginning of a new day. At the end of a day, the capillary was rinsed from the inlet to the outlet side 
with $0.2 \mathrm{M} \mathrm{NaOH}$ and water for 5 min each with a pressure of $60 \mathrm{psi}$, and the capillaries were stored wet with the ends kept in water. Between runs the capillary was first rinsed with $0.2 \mathrm{M} \mathrm{NaOH}$ for $1 \mathrm{~min}$ with a pressure of 60 psi applied at the inlet end, followed by $0.2 \mathrm{M} \mathrm{NaOH}$ with applying simultaneously a pressure of 20 psi from the inlet to the outlet end and a constant current of $80 \mu \mathrm{A}$ for $1.5 \mathrm{~min}$. At the beginning of each run, the capillary was first conditioned from the inlet side with the initiator buffer by applying a pressure of $15 \mathrm{psi}$ for $1.5 \mathrm{~min}$ and then with the run buffer for $2 \mathrm{~min}$ by applying the same pressure. All Tf isoforms were detected within $12 \mathrm{~min}$ and the total time interval required for analysis and conditioning was $22 \mathrm{~min}$.

\subsection{CDT determination with the \%CDT TIA test and determination of serum alcohol}

CDT was determined with the turbidimetric immunoassay \%CDT TIA of AxisShield (distributed through Bio-Rad, Hercules, CA, USA) according to the recommendations of the kit manufacturer using the Cobas Mira plus (Roche Diagnostics, Rotkreuz, Switzerland). A CDT value $<2.6 \%$ of total Tf was considered to be within the normal range. Serum alcohol was determined on the Vitros 250 using the Vitros ALC slide technology (Ortho Clinical Diagnostics, Neckargemund, Germany).

\section{Results and Discussion}

\subsection{Precision performance and quality control}

It was shown in a previous publication that the CEofix-CDT kit, employed according to the instructions of the manufacturer, resulted in an excellent precision performance. The bottleneck of the assay, however, was identified to be the incomplete separation of disialo- and trisialo-Tf, with the resolution between these two peaks being typically $<1.3$ for sera of healthy individuals and alcohol abusers [17]. Without changing the capillary dimensions and the composition of the running buffer, baseline separation of di- and trisialo-Tf could be achieved by lowering the applied voltage and the temperature of the capillary cartridge [20], resulting in the optimized method described in this manuscript.

The precision performance of the optimized method in regard to migration times of $\mathrm{Tf}$ isoforms and quantification of single $\mathrm{Tf}$ isoforms and CDT was evaluated. Customary intra-day and inter-day precision data were calculated for a serum of a 
healthy volunteer containing a CDT amount below the cut-off value, referred to as "sample low", and for a patient serum with an elevated CDT level, referred to as "sample high". Electropherograms of the two sera are depicted in Fig. 1. For the intraday data, eight samples of each serum were prepared separately and analyzed in one series. For the gathering of the inter-day data, one sample of each serum was prepared and analyzed on eight consecutive working days. The precision data are presented in Tables 1 and 2. RSD values for the intra-day precision of migration times of all Tf peaks were found to range between 0.18 and $0.29 \%$. Corresponding values for the inter-day data were between 0.58 and $0.66 \%$, values that are more than two-fold lower compared to those obtained employing the instrumental conditions proposed by the manufacturer referred to as the standard method [17]. In agreement with previous findings [17], RSD values for the quantitative determination of single Tf isoforms and CDT were found to be dependent on the isoform level, with the lowest RSD values $(<0.26 \%)$ for tetrasialo-

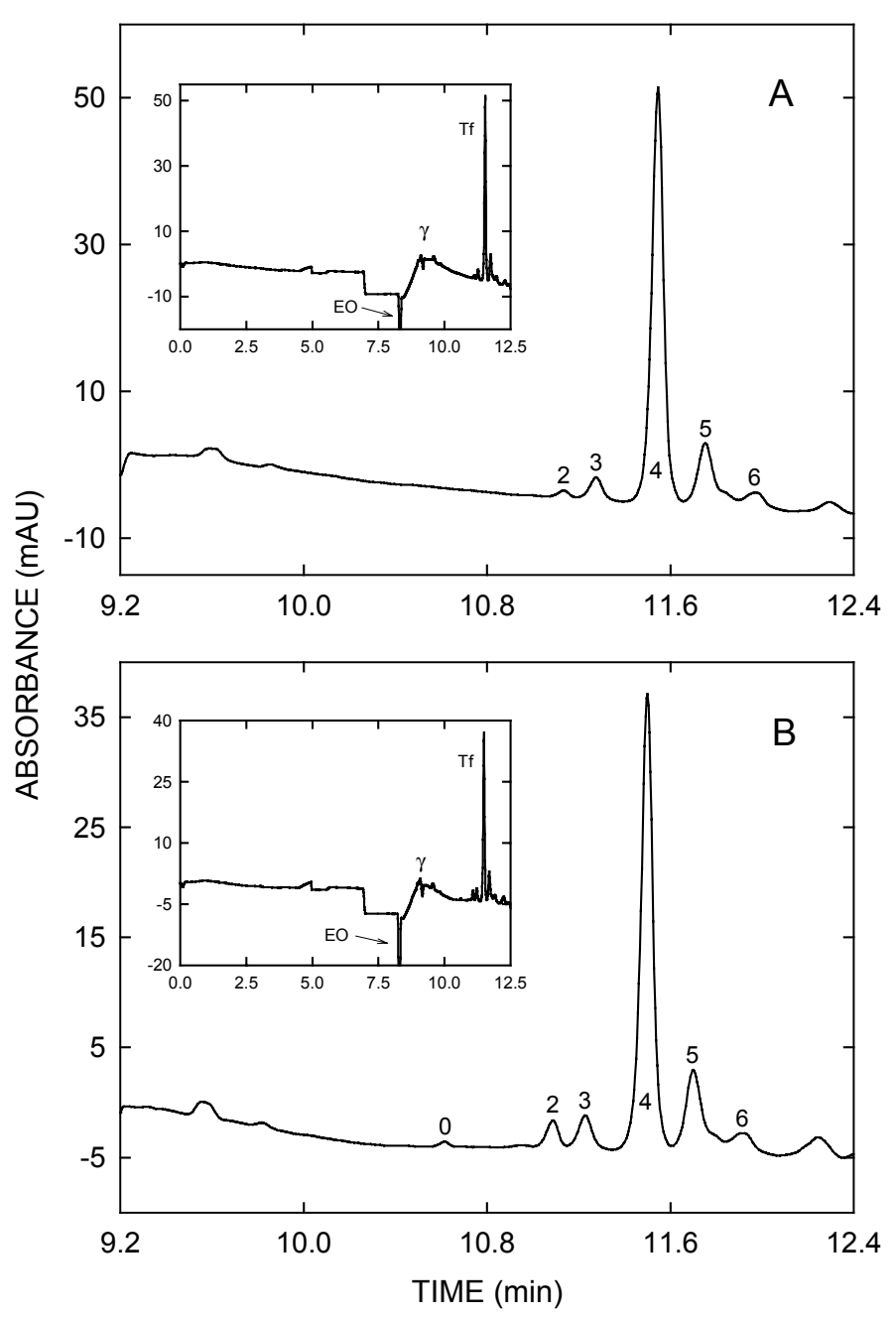

Figure 1: Typical CZE Tf patterns obtained with a sample containing (A) a normal CDT level of $1.05 \%$ and (B) an elevated CDT level of $4.6 \%$. The inserts depict the entire electropherograms. EO and $\gamma$ refer to electroosmosis peak and $\gamma$-region, respectively. Isoform levels are given in Table 1 and experimental conditions are provided in Sections 2.2 and 2.3. Key: 0: asialo-Tf, 2: disialo-Tf, 3: trisialo-Tf, 4: tetrasialo-Tf, 5: pentasialo-Tf, 6: hexasialo-Tf. 


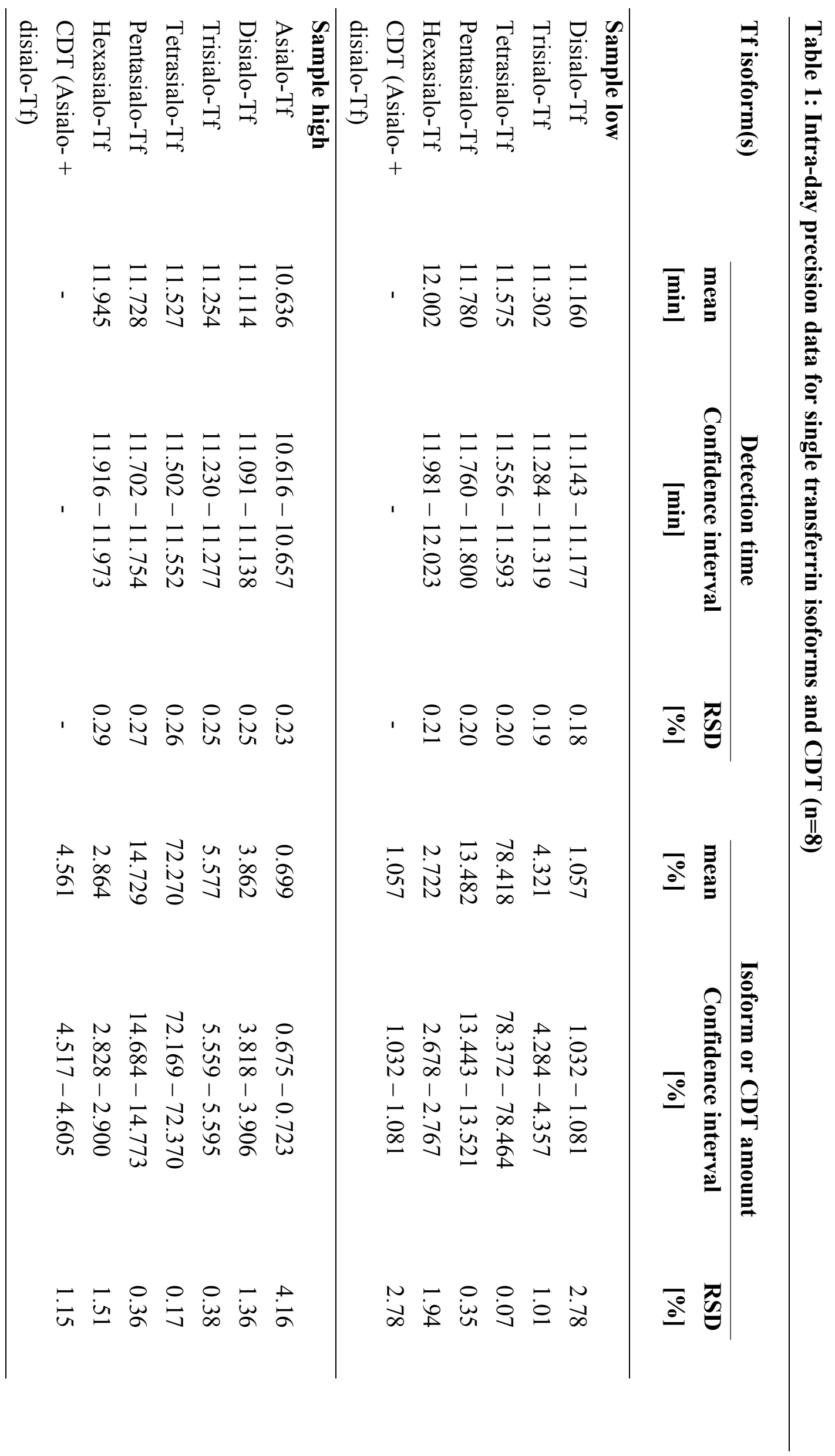




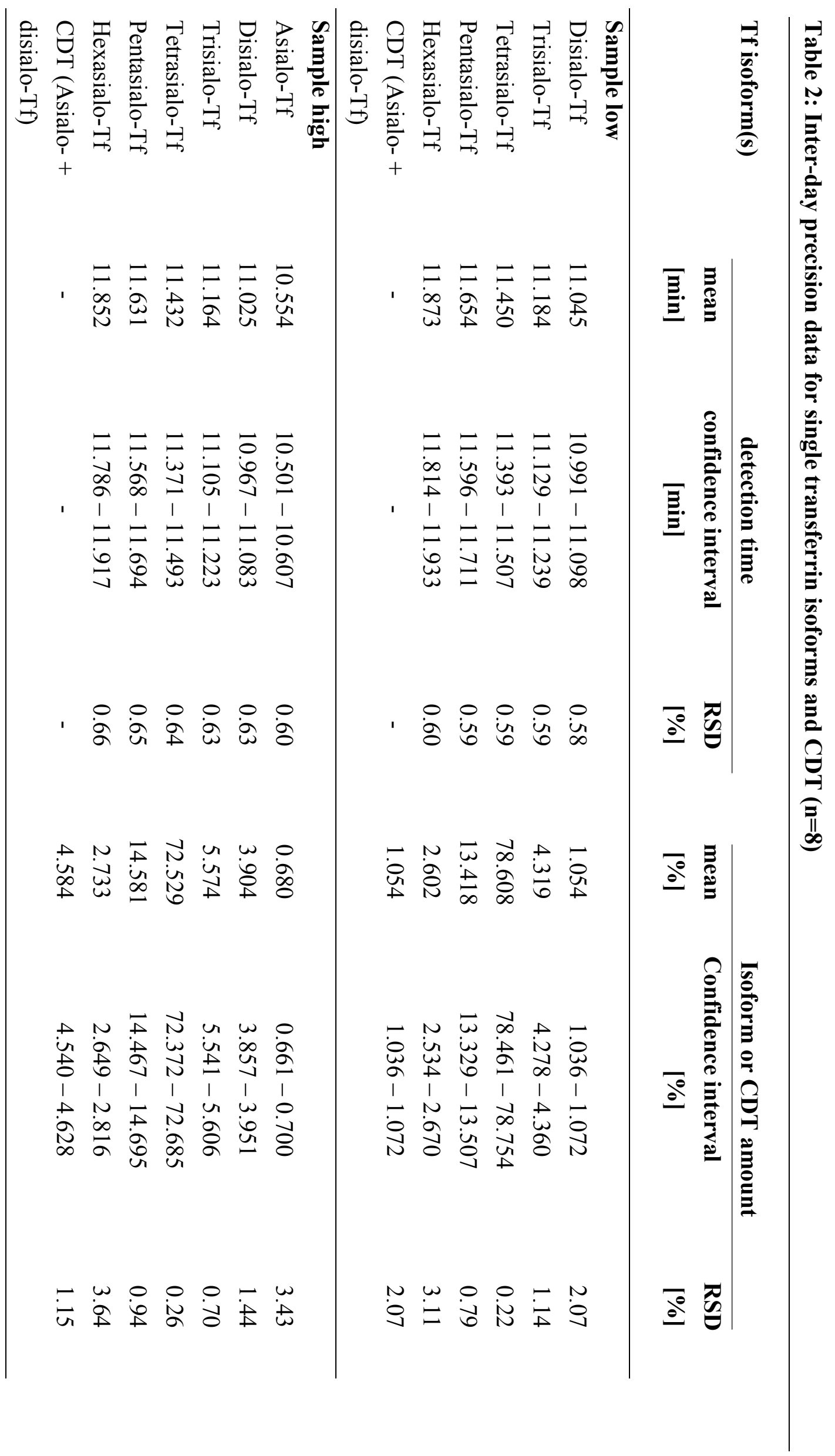


Tf and the highest for asialo-Tf $(<4.2 \%$ ) (Tables 1 and 2$)$. The observed RSD values are $2-3.5$ times lower than those obtained with the standard method $[17,20]$.

The quantification and detection limits for single isoforms were assessed for asialo- and disialo-Tf and determined to be $0.10 \%$ and $0.05 \%$, respectively. Peaks leading to values lower than $0.10 \%$ were not accounted for the determination of CDT. For the "sample low" with a CDT level of about $1.06 \%$, no asialo- and monosialo-Tf could be detected. Intra-day and inter-day RSD values for CDT were found to be 2.78 and $2.07 \%$, respectively. No monosialo-Tf could be detected in the "sample high" whose CDT level was determined to be about $4.6 \%$ and intra-day and inter-day RSD values were found to be $1.15 \%$ (Tables 1 and 2).

For internal quality control, a control sample stemming from the serum of a healthy volunteer was included in each set of runs. The monitored electropherogram did not exhibit an asialo-Tf peak. In the first year of routine use of the assay, 62 sets of samples were analyzed. The same capillary was employed during the entire time period during which a total of about 1000 analyses (total of patient, control and research samples) were analyzed and there were no instrumental problems. The mean (RSD) disialo-Tf or CDT level calculated from the 62 values was determined to be $1.01 \%$ (3.79\%). Similarly, the mean (RSD) trisialo-Tf amount in the same sample was found to be $4.19 \%(2.24 \%)$. These data document both the excellent precision of the assay and the above discussed dependence of isoform level on the RSD value. Thus, the data generated thus far demonstrate that the optimized method is very robust and reproducible.

\subsection{Determination of CDT in patient samples}

As discussed in detail elsewhere [20], the CDT reference limits were calculated by non-parametric methods from the $2.5^{\text {th }}$ and $97.5^{\text {th }}$ percentiles of the disialo-Tf reference values of 54 reference samples (asialo-Tf was not detected in these samples) that stemmed from individuals with no or moderate $(\leq 20 \mathrm{~g}$ ethanol per day) alcohol consumption. For the optimized separation conditions, the mean of the CDT values was $1.13 \%$ (SD: $0.18 \%$; median: $1.11 \%$; range: $0.80-1.59 \%$ ) and the upper $(90 \%$ confidence interval) and lower (90\% confidence interval) reference limits were determined to be $1.57 \%(1.50-1.63 \%)$ and $0.81 \%(0.74-0.88 \%)$, respectively. Based on these data, the threshold value used for routine analysis was set to $1.7 \%$ which is essentially the mean $+3 \mathrm{SD}$. 
The optimized CZE assay was introduced as routine method for the determination of CDT levels in patient sera in our departmental analytical laboratory. During the first year of operation, more than 600 samples were analyzed. The majority of samples provided straightforward patterns such as those depicted in Fig. 1. Sera whose electropherograms showed unusual peak distributions were reanalyzed after immunosubtraction of Tf (Fig. 2, lower graphs) and/or identified as discussed previously [17] (Fig. 3). A total of 6 sera revealed heterozygous Tf-BC variants (Fig. 3A) and one serum showed a heterozygous Tf-CD variant. 5 samples comprised elevated peaks in the $\gamma$-globulin region (Fig. 3B), and specimens with elevated C-reactive protein (CRP) levels were monitored with a peak detected shortly before disialo-Tf (Fig. 3C). Furthermore, for selected samples stemming from patients treated at the hepatology outpatient clinic, a broad interference under the Tf pattern was detected (Fig. 3D). Elevated immunoglobulin A levels are assumed to be the reason for the interference.

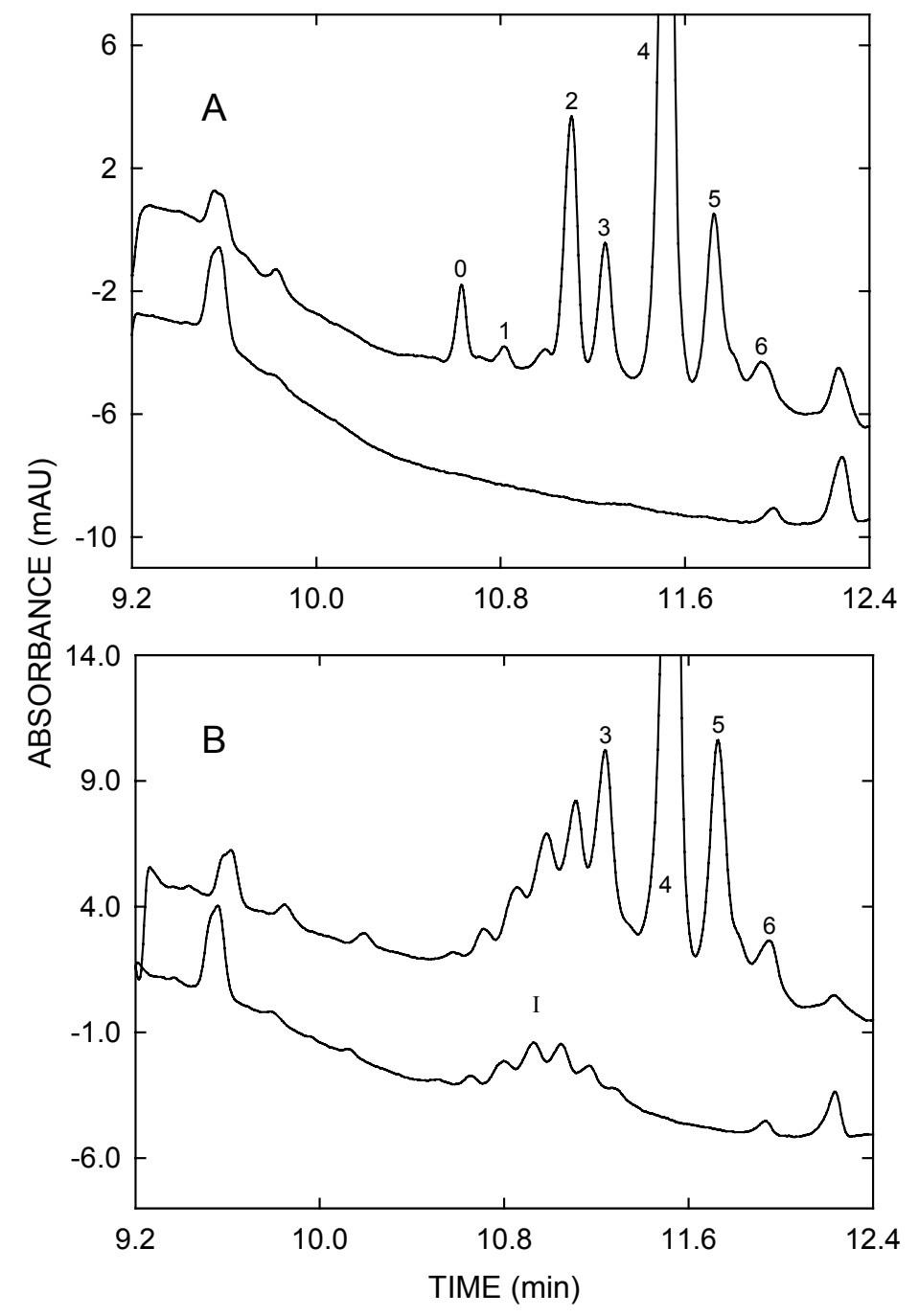

Figure 2: Electropherograms before (upper graphs) and after (lower graphs) immunosubtraction of Tf obtained with (A) the serum of an alcohol abuser that revealed the presence of monosialo-Tf and (B) the serum of a patient comprising an unknown, multiplepeak interference (marked with I in lower graph). The CDT level of the serum whose data are depicted in panel A was determined to be $18.77 \%$ (14.28\% of disialo-Tf, $3.59 \%$ of asialo-Tf and $0.90 \%$ of monosialo-Tf). For experimental conditions refer to Sections 2.2 and 2.3. Key: 0: asialo-Tf, 2: disialo-Tf, 3: trisialo-Tf, 4: tetrasialo-Tf, 5: pentasialo-Tf, 6: hexasialo-Tf. 

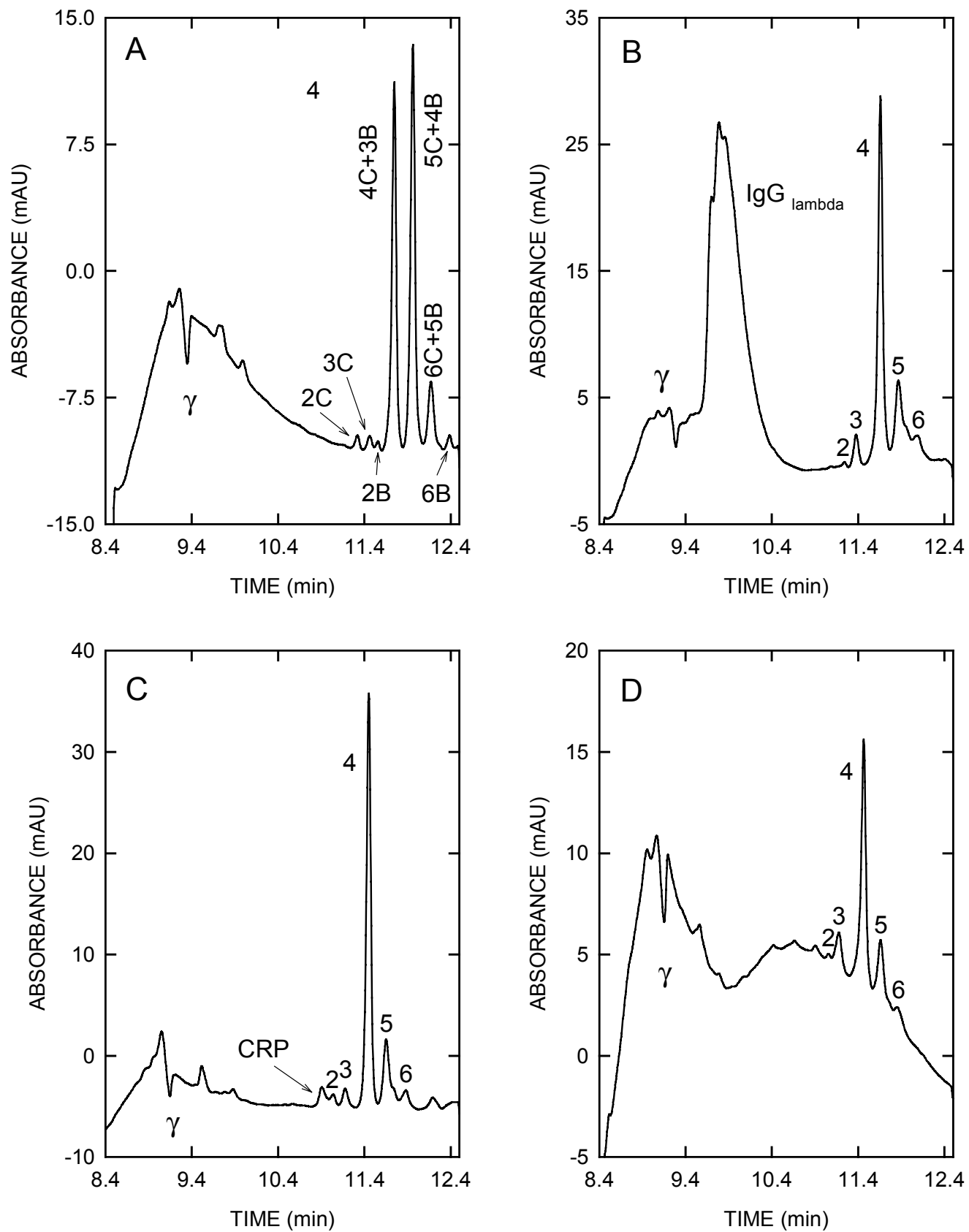

Figure 3: Unusual CZE electropherograms obtained for (A) a heterozygous Tf-BC variant, (B) a sample with a large peak in the $\gamma$-region ( $\operatorname{IgG}_{\text {lambda }}$, same sample as for Fig. 6A in [15]), (C) a serum with an elevated CRP level of $93 \mathrm{mg} / \mathrm{L}$, and (D) a serum with a broad interference in the Tf region. For experimental conditions refer to Sections 2.2 and 2.3. Key: 0: asialo-Tf, 2: disialo-Tf, 3: trisialo-Tf, 4: tetrasialo-Tf, 5: pentasialo-Tf, 6: hexasialo-Tf. 
However, our efforts in removing the interfering compounds failed thus far and more work is required for elimination and identification of this problem. Two sera of one patient drawn 6-month apart revealed the presence of an unknown interference that prevented the determination of CDT with this assay (Fig. 2B).

The levels of asialo- and disialo-Tf of part of the 589 patient samples are depicted in Fig. 4 (presented in the order of increasing disialo-Tf values) and the frequency diagrams of disialo-Tf and CDT levels of all 589 samples are shown in Fig. 5. Disialo-Tf levels ranged between 0.31 and $14.41 \%$ (mean: $1.52 \%$, median: $1.18 \%$ ), asialo-Tf was detected in 71 samples whereas asialo-Tf was quantitated in 49 sera (range: $0.14-3.63 \%$, mean: $0.85 \%$, median: $0.41 \%$ ). CDT levels were found to be between 0.31 and $18.04 \%$ (mean: $1.59 \%$, median: $1.18 \%$ ). In serum samples revealing CDT levels below the upper reference limit determined for this assay $(1.70 \%$, total of 479 samples), no asialo-Tf was detected. The same was essentially found to be true for samples with disialo-Tf levels below $2.30 \%$ (up to sample 522). Up to this level, small asialo-Tf peaks could be monitored in 11 (quantitated in 2) sera only (Fig. 4). Asialo-Tf was detected in 60 (quantitated in 47) of the 67 samples with disialo-Tf levels $>2.30 \%$. The fact that in some cases even in sera with CDT levels up to $3.71 \%$ no asialo-Tf was

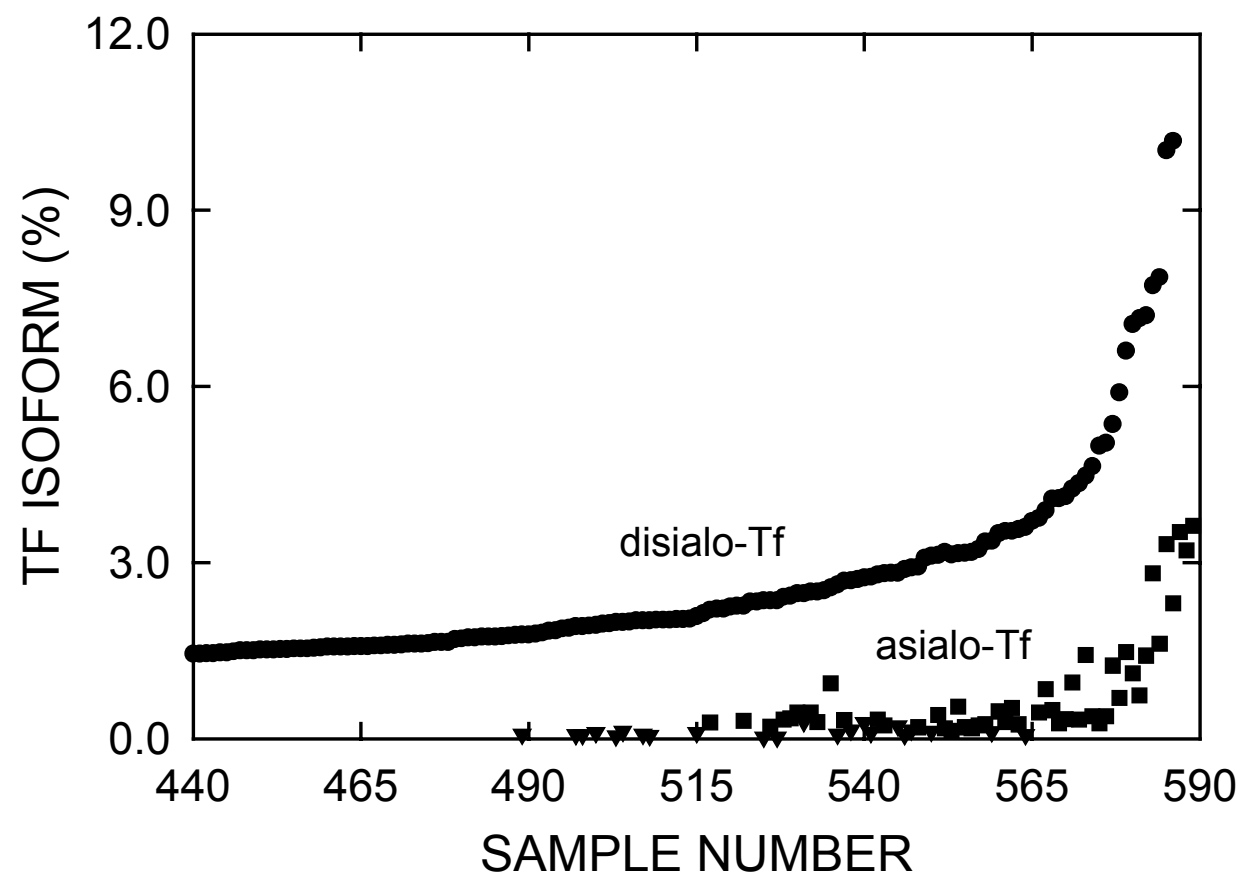

Figure 4: Disialo-Tf (circles) and asialo-Tf (triangles for detectable peaks between 0.05 and $0.1 \%$ and squares for quantitated peaks $\geq 0.1 \%$ ) levels of the samples 440 to 589 presented in the order of increasing disialo-Tf values. 
present may be explained by the inter-individualvariation of the response in CDT isoform formation $[21,22]$.

In a patient sample with a CDT value of $18.77 \%(14.28 \%$ of disialo-Tf, $3.59 \%$ of asialo-Tf), monosialo-Tf was detected with a relative amount of $0.90 \%$ (Fig. 2A, upper graph). Based on the data obtained after immunosubtraction (Fig. 2A, lower graph), and from the pattern change obtained after neuraminidase treatment [17], the peak detected after $10.81 \mathrm{~min}$ (relative migration time compared to tetrasialo- $\mathrm{Tf}$ of 0.939) was assigned to monosialo-Tf. Mårtensson et al. [23] reported monosialo-Tf to be present in trace amounts in the sera of social drinkers. These monosialo-Tf concentrations are believed to be below the detection limit of our CZE assay. Furthermore, the monosialo-Tf increase during chronic alcohol consumption is reported to be much less pronounced compared to the increase of the other CDT isoforms [23]. These data are in good agreement with our findings as no peak could be attributed to monosialo-Tf in samples with CDT levels $<13.3 \%$. It can be speculated that

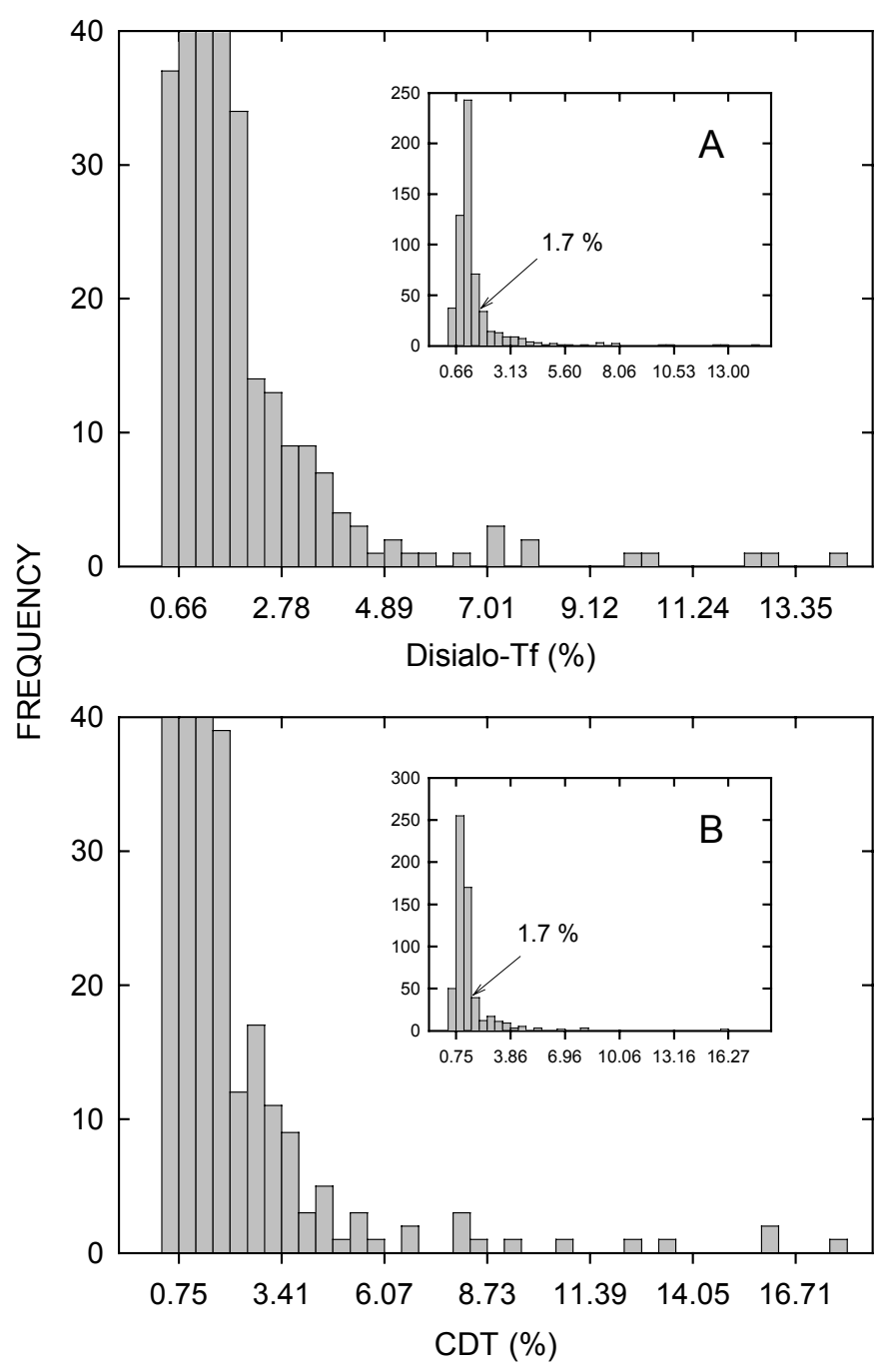

Figure 5: Frequency diagrams of (A) disialo-Tf and (B) CDT levels of the 589 patient samples. The widths of the bars are 0.35 and 0.44 $\%$, respectively. 
monosialo-Tf reaches the detection limit of the employed CZE assay only after chronic intake of highest amounts of alcohol, producing extremely high values of asialo- and disialo-Tf.

\subsection{Long-term CDT monitoring}

Although the value of CDT for identifying high risk drinkers and alcohol abusers is still discussed controversely, with reported test sensitivities and specificities depending on the investigated patient collective and control group, the study design and the analytical principle employed, CDT is widely used for assisting the diagnosis of alcohol abuse and alcohol dependence [2,3,24-26]. Longitudinal CDT monitoring of individuals with a history of alcohol abuse is important for the early detection of relapse drinking after abstinence and in legal cases [4,25]. The use of an individual cut-off drawn from an abstinent or drinking baseline of the monitored person, instead of a common upper reference limit derived from the statistical distribution of a reference sample group (see above), has been recommended for that purpose. The biological variation of CDT values between individuals can thereby be overcome, while the intraindividual serum CDT levels are reported to be fairly constant in teetotallers and social drinkers [2,21,22,25,27-29].

CDT of one patient was monitored with the turbidimetric immunoassay $\%$ CDT TIA of Axis-Shield over an extended period of time (for values refer to the last column in Table 3). Although alcohol could not be determined in these samples, CDT was found to be markedly elevated at days $31,36,43$ and 50 , with CDT values being above the upper reference limit of $2.6 \%$ adopted for this immunoassay according to the manufacturer's instructions and above the $3.0 \%$ treshold value often employed in clinical practice [30]. As this increase was unexpected, the samples were subjected to confirmation analysis by CZE. The time courses of the thereby determined levels of single CDT isoforms and CDT are presented in Fig. 6 and the values of all Tf isoforms and CDT are summarized in Table 3. Fig. 7 depicts selected electropherograms. Using CZE, the four samples in question were also found to be positive. Linear regression analysis of the two sets of data revealed a linear relationship with CDT(\%CDT TIA) $=$ $0.744 \times \operatorname{CDT}(\mathrm{CZE})+1.256(\mathrm{r}=0.990, \mathrm{n}=18)$.

With the CZE-based confirmation analyses data, detailed information about the temporal behavior of all $\mathrm{Tf}$ isoforms is obtained. The first two samples, drawn within 10 days, showed almost identical CDT values $(0.98 \%$ and $0.96 \%)$ and no asialo-Tf (Table 3 ). These CDT values are similar to the mean of the reference samples (see above), are 


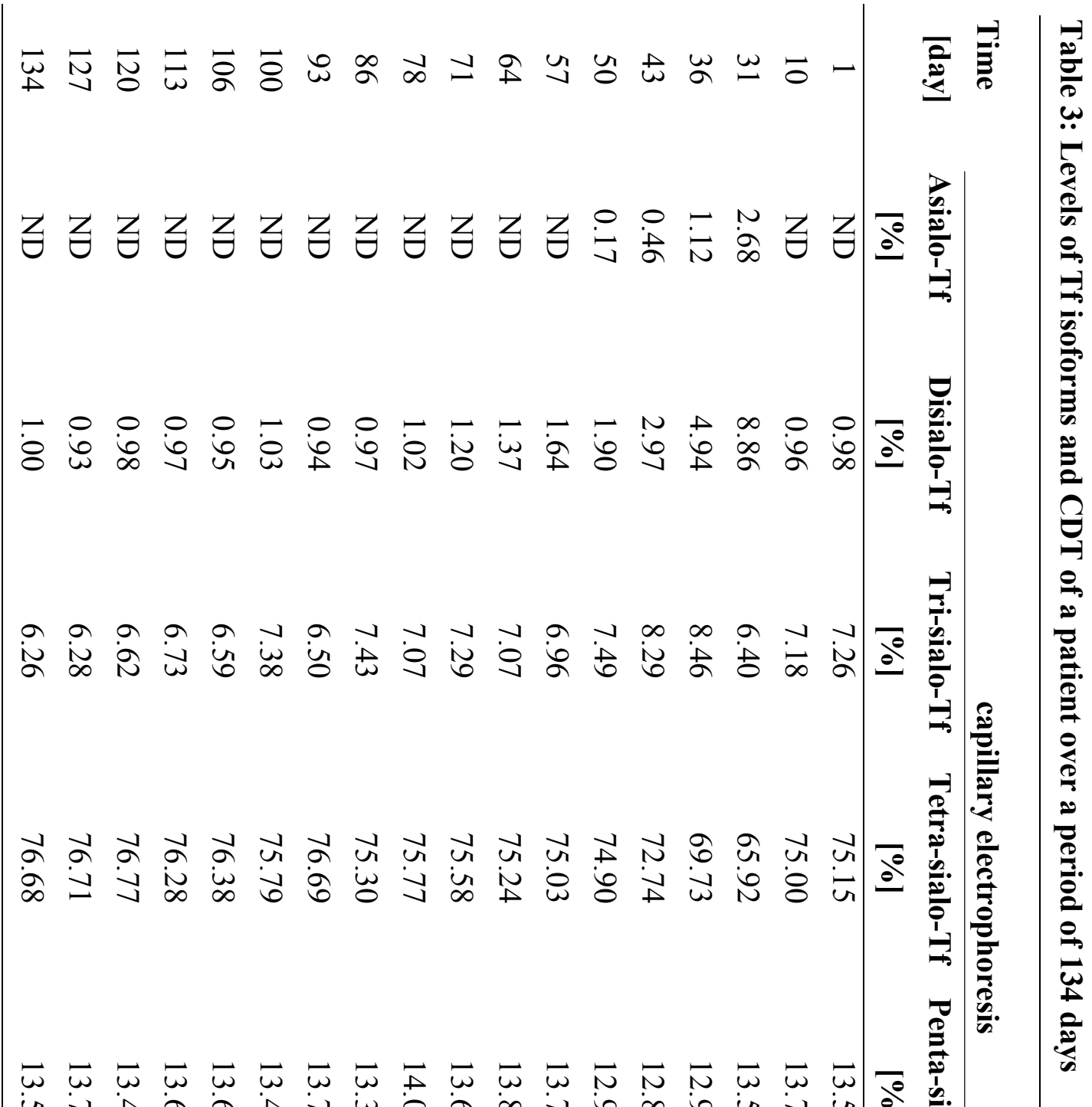

$$
\begin{aligned}
& \text { 出 } \overrightarrow{0} \text { b }
\end{aligned}
$$

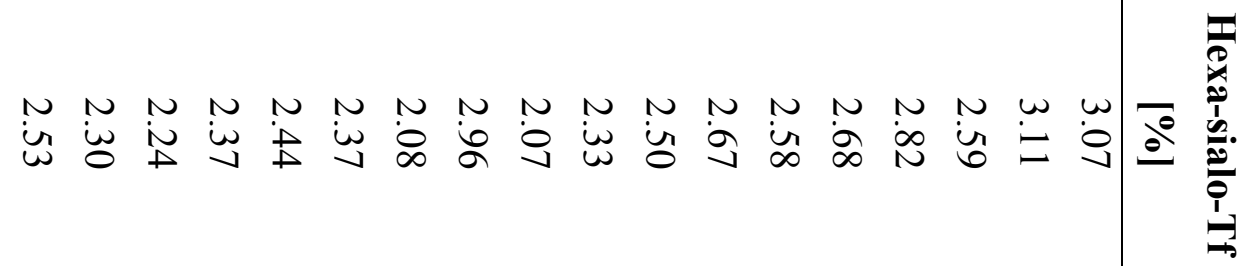

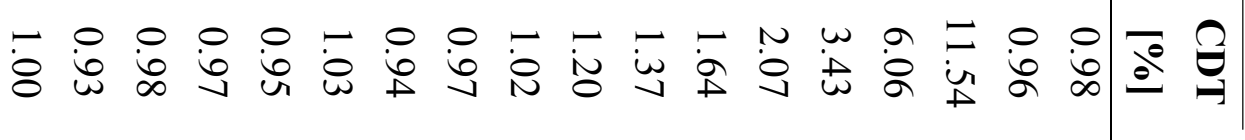

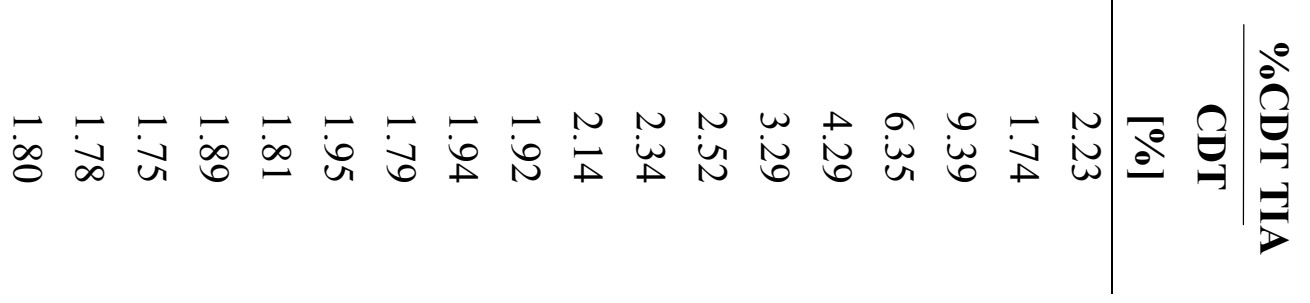


far below the upper reference limit of the method $(1.70 \%)$ and are considered to represent the baseline value of this patient (see below). Three weeks later, about a twelve-fold increase of CDT (11.54\%) with detectable asialo-Tf (2.68\%) was monitored. Thereafter, a continuous decrease of the levels of asialo-Tf, disialo-Tf and thus CDT was noticed during the following 7 weeks, reaching the previous baseline value of CDT at day 78 (Fig. 6A). CDT levels above the cutoff value of $1.70 \%$ were obtained up to day 50. Asialo-Tf was detected in the same time interval and monosialoTf could not be detected at any time. From days $57-71$, CDT was below the upper reference limit of the assay $(1.70 \%)$ but above the individual patient's baseline value. The level of asialo-Tf was found to decrease exponentially (Fig. 6B). Using a PC compatible, one-compartment model for elimination of an exogenous compound (Excel 97 SR2, PK functions for Microsoft Excel, www.boomer.org), the apparent half life of the elimination was determined to be 4.86 days. Disialo-Tf and CDT serum levels did not decrease in the same manner (Fig. 6B) and apparent half lifes were estimated to be

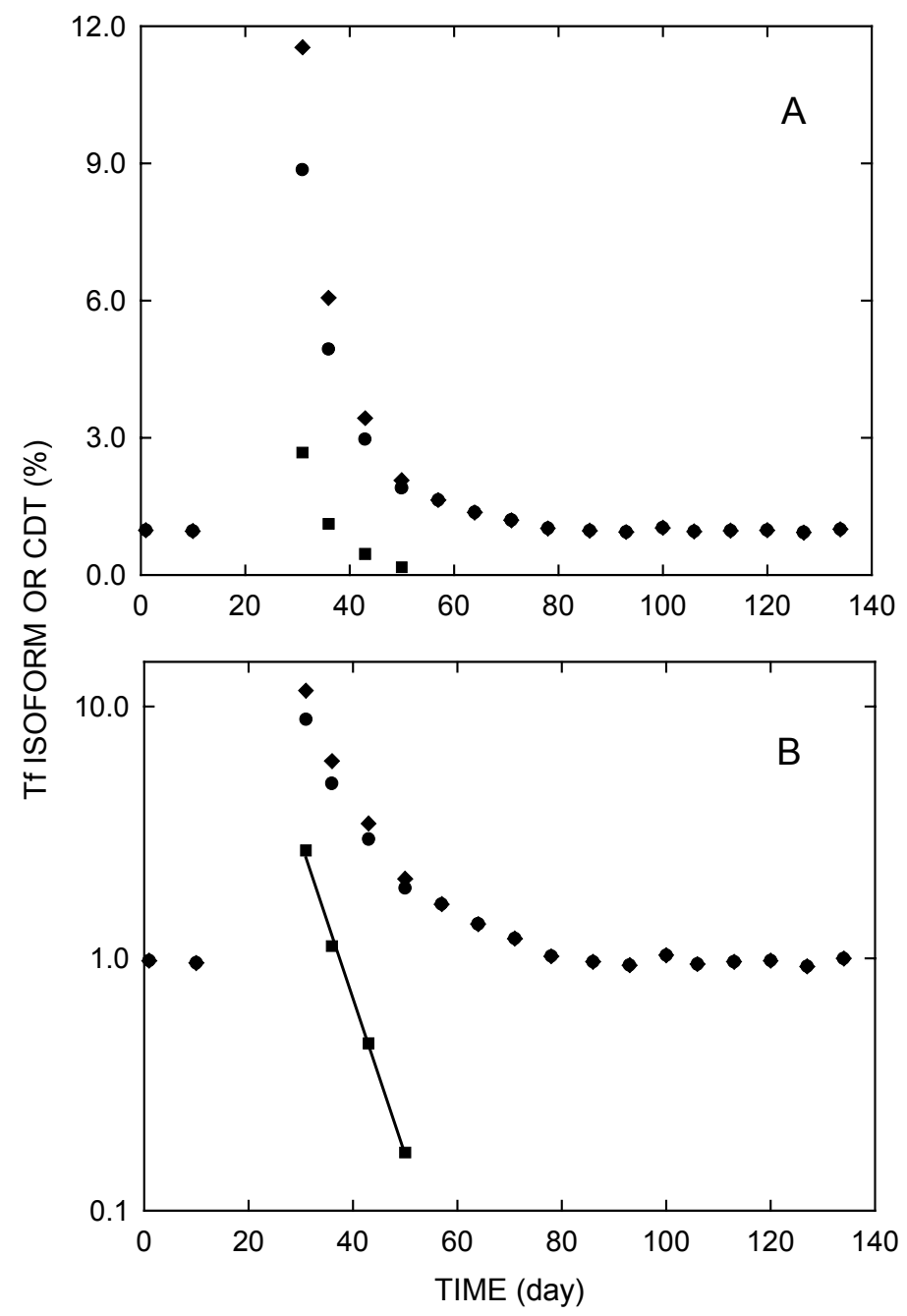

Figure 6: CZE data of long-term CDT monitoring: Disialo-Tf (circle), asialo-Tf (square) and CDT (diamond) levels of a patient determined by CZE during a 134day period. The data in panel $\mathrm{B}$ are the same as those of panel A but presented in a semi-log format. The straight line in panel $B$ represents the regression line for asialo-Tf $(y=-0.0619 x+2.3196$, $r=0.9984)$. 
7.24 and 6.74 days, respectively. These values compare well with those reported in the literature [1-4,31].

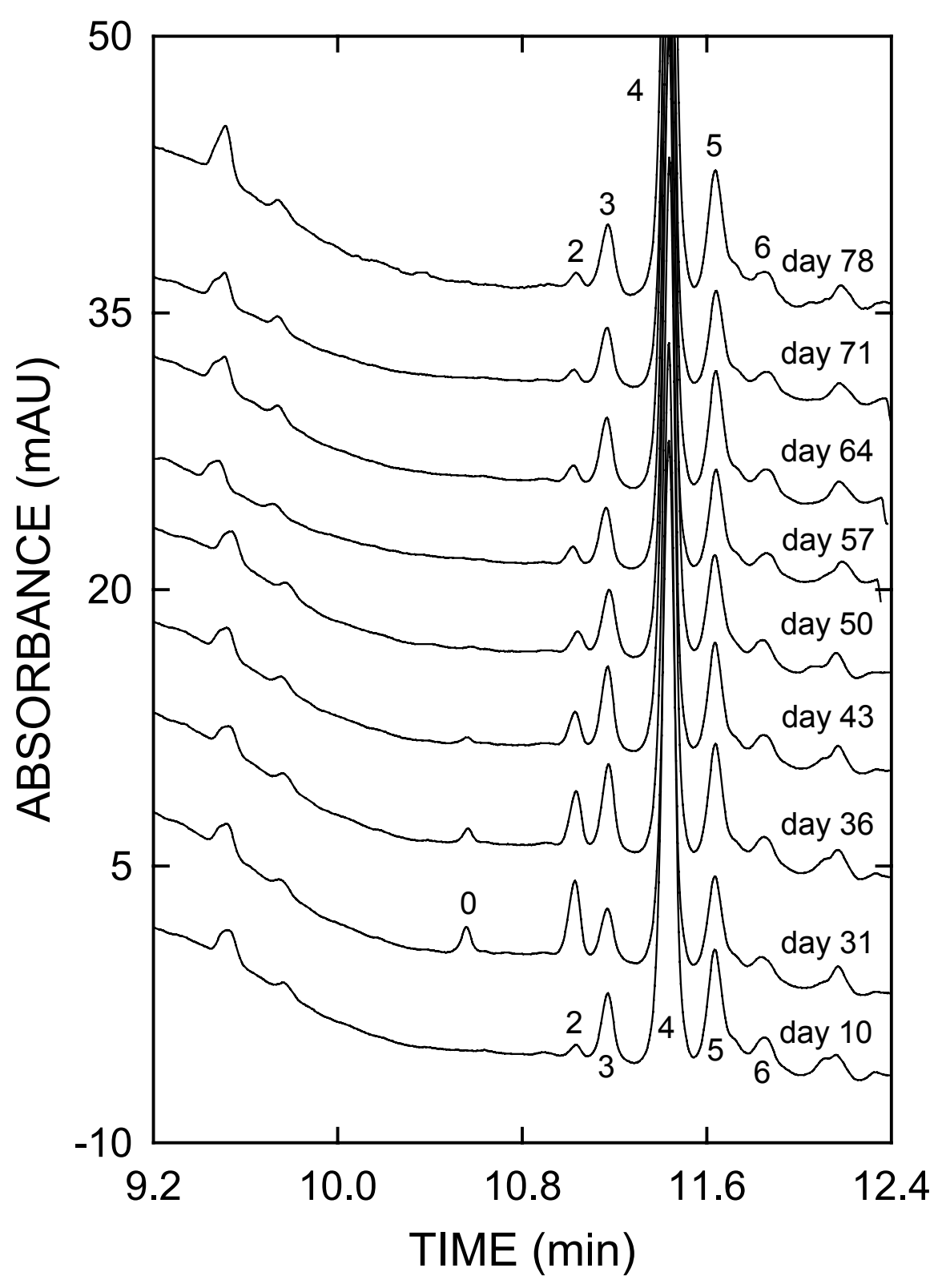

Figure 7: Selected electropherograms for the data of Fig. 6. The data are presented with y-axis offsets and the $\mathrm{x}$-axis being adjusted for equal appearance of tetrasialo-Tf. For experimental conditions refer to Sections 2.2 and 2.3. Key: 0: asialo-Tf, 2: disialo-Tf, 3: trisialo-Tf, 4: tetrasialo-Tf, 5: pentasialo-Tf, 6 : hexasialo-Tf. 
The mean value of the 11 baseline values (samples at days 1, 10, and 78 to 134) was calculated to be $0.98 \% \pm 0.03 \%$. The considerably smaller variation compared to that reported by Borg et al. [28] can at least partly be attributed to the much higher reproducibility of the CZE assay (see section 3.1.) compared to that of the immunoassay for which an assay precision of $10 \%$ is reported. Adopting the method described in [28] for identification of relapse drinking, namely an increase of CDT from the minimum or baseline value of three or four times the coefficient of variation of the baseline values, would lead to a cut-off level for this patient of 1.07 to $1.10 \%$. These findings are in good agreement with the data of Anton et al. who reported a mean elevation of CDT levels of $10 \%$ among relapse drinkers during a twelve week outpatient trial [22].

Trisialo-Tf values varied between 6.26 and $8.46 \%$ (mean: $7.07 \%$, RSD: 8.77 $\%)$. For the 11 samples with baseline CDT values, mean and RSD were determined to be $6.85 \%$ and $6.28 \%$, respectively. The highest trisialo-Tf level was detected for the sample at day 36 with its value being $23.5 \%$ above the mean value of the samples with baseline CDT. Whether this small increase can be attributed to an effect related to the alcohol intake is unclear. In agreement with the literature, no significant change in the trisialo-Tf levels could otherwise be observed $[8,32,33]$. Not surprisingly, compared to disialo-Tf, tetrasialo-Tf and to a much smaller extent pentasialo-Tf showed a reciprocal change of the relative amount. The lowest value of tetrasialo-Tf (13.3\% below the 76.09 $\%$ mean value of the 11 samples with baseline CDT values) was noticed when CDT was highest (day 31), while the lowest value of pentasialo-Tf (5.6 \% less than the $13.63 \%$ mean value of samples revealing baseline values of CDT) was determined with a time shift of twelve days (day 43). Hexasialo-Tf did not change significantly during the entire episode.

There is still some confusion about the daily amount of ethanol and the duration of regular alcohol intake needed to elevate CDT. Reports claiming that daily intake of $50-80 \mathrm{~g}$ pure ethanol over a period of at least one week resulted in increased CDT levels $[1-4,7,34]$ are contradicted by drinking experiments with healthy individuals, concluding that an intake of up to $60-80 \mathrm{~g}$ ethanol per day for three weeks did not increase CDT [35] or showed a significant but only moderate elevation without reaching the upper reference limit for most individuals [36]. It was speculated that the response of CDT to alcohol consumption of subjects with no drinking history may be different from that of individuals with alcohol dependence, leading to sensitization caused by regular drinking [3,22,35,37,38]. The huge increase of CDT found in the monitored patient was unexpected and can only be a result of very high alcohol consumption between days 10 and 31 . 


\section{Concluding remarks}

Using the CEofix-CDT reagents and the P/ACE MDQ under optimized instrumental conditions $\left(20 \mathrm{kV}\right.$ and $30^{\circ} \mathrm{C}$ ) is demonstrated to provide outstanding assay precision for the determination of CDT in human serum. Compared to the conditions recommended by the kit manufacturer $\left(28 \mathrm{kV}\right.$ and $\left.40{ }^{\circ} \mathrm{C}\right)$, resolution between diasiloand trisialo-Tf is improved and complete such that the CDT relevant $\mathrm{Tf}$ isoforms can be quantitated precisely. Intra-day and inter-day precision data for asialo- and disialo-Tf were found to be isoform level dependent. For isoform levels of about $0.7 \%, 1.0 \%$ and $4.0 \%$, RSD values $(\mathrm{n}=8)$ were determined to be $<4.2 \%,<3.0 \%$ and $<1.5 \%$, respectively. During the first year of operation under routine conditions, more than 600 patient samples were analyzed in a total of 62 sets of runs and without changing the capillary. The control sample analyzed in this one-year period $(n=62)$ revealed RSD values of $3.79 \%$ and $2.24 \%$ for mean isoform levels of $1.01 \%$ and $4.19 \%$, respectively. Except for selected samples received from patients being treated at the hepatology outpatient clinic, interference-free Tf patterns were detected. Asialo-Tf was not detected in control sera and in patient sera with a CDT level $<1.70 \%$, but became detectable in $89.6 \%$ of sera with $>2.3 \%$ disialo-Tf. Monosialo-Tf was only detected in a few sera containing $>13.3 \%$ CDT. The optimized CZE assay was applied to confirm positive CDT results produced by an immunoassay during long-term monitoring of a patient which led to the determination of the elimination kinetics of asialo-Tf, disialo-Tf and CDT after an episode of high alcohol consumption. Apparent elimination half lifes could thereby be estimated to be 4.86, 7.24 and 6.74 days, respectively. The optimized CZE assay with an upper reference limit for CDT of $1.70 \%$ represents a very attractive alternative to HPLC. It features simpler sample preparation, faster analysis time, higher isoform resolution and much simpler column reconditioning compared to the most recent improved HPLC approach [12]. The CZE approach can thus be employed for both, screening and confirmation analysis alike and fulfills the requirements of a reference method for CDT. The improved assay could also be used for legal cases in forensics.

\section{Acknowledgements}

The authors acknowledge the stimulating discussions with Prof. B.H. Lauterburg and the calculation of the kinetic data by Dr. S. Russmann. This work was supported by Analis (Namur, Belgium), the Liver Foundation (Bern, Switzerland) and the Swiss National Science Foundation. 


\section{References}

[1] Musshoff, F., Daldrup, Th., J. Chromatogr. B 1998, 713, 245-264.

[2] Tagliaro, F., Bortolotti, F., Crivellente, F., Cittadini, F., Forensic Sci. Rev. 2000, $12,134-148$.

[3] Sharpe, P.C., Ann. Clin. Biochem. 2001, 38, 652-664.

[4] Musshoff, F., J. Chromatogr. B 2002, 781, 457-480.

[5] Stibler, H., Kjellin, K.G., J. Neurol. Sci. 1976, 30, 269-285.

[6] Stibler, H., Allgulander, C., Borg, S., Kjellin, K.G., Acta med. Scand. 1978, 204, 49-56.

[7] Stibler, H., Borg, S., Allgulander, C., Acta med. Scand. 1979, 206, 275-281.

[8] Arndt, T., Clin. Chem. 2001, 47, 13-27.

[9] Meerkerk, G-J., Njoo, K.H., Bongers, I.M.B., Trienekens, P., van Oers, J.A.M., Alcohol. Clin. Exp. Res. 1998, 22, 908-913.

[10] Jeppsson, J.O., Kristensson, H., Fimiani, C., Clin. Chem. 1993, 39, 2115-2120.

[11] Helander, A., Eriksson, G., Stibler, H., Jeppsson, J.-O., Clin. Chem. 2001, 47, 1225-1233.

[12] Helander, A., Husa, A., Jeppsson, J.-O., Clin. Chem. 2003, 49, 1881-1890.

[13] Oda, R.P., Landers, J.P., Electrophoresis 1996, 17, 431-437.

[14] Crivellente, F., Fracasso, G., Valentini, R., Manetto, G., Riviera, A.P., Tagliaro, F., J. Chromatogr. B 2000, 739, 81-93.

[15] Giordano, B.C., Muza, M., Trout, A., Landers, J.P., J. Chromatogr. B 2000, 742, 79-89.

[16] Lanz, C., Kuhn, M., Bortolotti, F., Tagliaro, F., Thormann, W., J. Chromatogr. A $2002,979,43-57$.

[17] Lanz, C., Marti, U., Thormann, W., J. Chromatogr. A 2003, 1013, 131-147.

[18] Legros, F.J., Nuyens, V., Minet, E., Emonts, P., Zouaoui Boudjeltia, K., Courbe, A., Ruelle, J.-L., Colicis, J., de L'Escaille, F., Henry, J.-P., Clin. Chem. 2002, $48,2177-2186$.

[19] Legros, F.J., Nuyens, V., Baudoux, M., Zouaoui Boudjeltia, K., Ruelle, J.-L., Colicis, J., Cantraine, F., Henry, J.-P., Clin. Chem. 2003, 49, 440-449.

[20] Lanz, C., Thormann, W., Electrophoresis 2003, 24, 4272-4281.

[21] Anton, R.F., Sillanaukee, P., Alcohol. Clin. Exp. Res.1996, 20, 54A-56A.

[22] Anton, R.F., Moak, D.H., Latham, P., Alcohol. Clin. Exp. Res. 1996, 20, 841846. 
[23] Mårtenson, O., Härlin, A., Brandt, R., Seppä, K., Sillanaukee, P., Alcohol. Clin. Exp. Res. 1997, 21, 1710-1715.

[24] Scouller, K., Conigrave, K.M., Macaskill, P., Irwig, L., Whitfield, J.B., Clin. Chem. 2000, 46, 1894-1902.

[25] Anton, R.F., Alcohol 2001, 25, 185-188.

[26] Reynaud, M., Schellenberg, F., Loisequx-Meunier, M.-N., Schwan, R., Maradeix, B., Planche, F., Gillet, C., Alcohol. Clin. Exp. Res. 2000, 24, 14141419.

[27] Voltaire Carlsson, A., Hiltunen, A.J., Beck, O., Stibler, H., Borg, S., Alcohol. Clin. Exp. Res. 1993, 17, 703-708.

[28] Borg, S., Helander, A., Voltaire Carlsson, A., Högström Brandt, A.-M., Alcohol. Clin. Exp. Res. 1995, 19, 961-963.

[29] Huseby, N.-E., Bjordal, E., Nilssen, O., Barth, T., Alcohol. Clin. Exp. Res. 1997, 21, 1343-1346.

[30] Helander, A., Fors, M., Zakrisson, B., Alcohol Alcohol. 2001, 36, 406-412.

[31] Hultberg, B., Isaksson, A., Berglund, M., Alling, C., Alcohol. Clin. Exp. Res. $1995,19,452-456$.

[32] Dibbelt, L., Clin. Chem. 2000, 46, 1203-1205.

[33] Lipowski, M., Dibbelt, L., Seyfarth, M., Clin. Biochem. 2000, 33, 635-641.

[34] Stibler, H., Clin. Chem. 1991, 37, 2029-2037.

[35] Lesch, O., Walter, H., Antal, J., Heggli, D.-E., Kovacz, A., Leitner, A., Neumeister, A., Stumpf, I., Sundrehagen, E., Kasper, S., Alcohol and Alcoholism 1996, 31, 265-271.

[36] Salmela, K.S., Laitinen, K., Nyström, M., Salaspuro, M., Alcohol. Clin. Exp. Res. 1994, 18, 228-230.

[37] Allen, J.P., Litten, R.Z., Anton, R.F., Cross, G.M., Alcohol. Clin. Exp. Res. 1994 18, 799-812.

[38] Behrens, U.J., Worner, T.M., Lieber, C.S., Alcohol. Clin. Exp. Res. 1988, 12, 539-544. 


\section{Conclusions}

The evaluation of various dynamic capillary coatings revealed that this principle is highly effective for the CZE separation and quantitation of Tf isoforms and thus the determination of CDT in human serum. For all investigated buffer additives, optimal results were obtained employing $50 \mu \mathrm{m}$ I.D. fused-silica capillaries of $50 \mathrm{~cm}$ effective length and a capillary cartridge temperature of $20-25^{\circ} \mathrm{C}$. Minimal sample preparation was required, consisting only in the saturation of the serum Tf with iron. Using a boratebased running buffer of $\mathrm{pH} 8.3$ with either $3.0 \mathrm{mM}$ DAB or $0.02 \mathrm{mM}$ spermine as capillary conditioners provided results of remarkable similarity in terms of resolution, migration times and signal intensity for di-, tri-, tetra- and pentasialo-Tf. With the double coating asialo- to hexasialo-Tf could be separated and quantitated. Due to somewhat shorter migration times, higher signal intensity and better reproducibility, the approach with the double coating could be identified as being superior to the two amine-based coatings (chapter B.1).

Further investigations of the reagents producing the double coating revealed an impressive precision performance of the system. The precision was assessed according to the internationally accepted guidelines of the EP5-A protocol from NCCLS (National Committee for Clinical Laboratory Standards) [41]. Inter-day RSD values $(n=20)$ for migration times were found to be $<1.7 \%$. RSD values for the quantitation of CDT isoforms were determined to be dependent on the isoform level and ranged between 2.2 $\%$ and $17.6 \%$ (chapter B.2). The bottleneck of the assay run at $28 \mathrm{kV}$ and $40{ }^{\circ} \mathrm{C}$ was identified as being the separation between disialo- and trisialo-Tf, i.e. the cut between CDT and non-CDT Tf isoforms. Therefore, a systematic study about the separation of these two isoforms was undertaken. Optimization of the method without changing the buffer composition or the capillary dimensions was achieved via reduction of the cartridge temperature and the applied voltage. The final method, run at $20 \mathrm{kV}$ and 30 ${ }^{\circ} \mathrm{C}$, resulted in baseline separation of all $\mathrm{Tf}$ isoforms and exhibited outstanding precision performances. RSD values for CDT, calculated as the sum of asialo-, monosialo- and disialo-Tf, were found to be $<3 \%$ for a normal serum with a CDT value of $1.0 \%$, and $<1.5 \%$ for the serum of an alcohol abuser with a CDT level of $4.5 \%(n=8)$. Detection times were highly constant, with the RSD value being $<1 \%$, revealing that this assay was robust, reproducible and reliable and thus fulfilling the criteria for the introduction of this assay into routine analysis (chapters B.3 and B.4). The health associated 
reference interval for CDT was established and found to be dependent on the instrumental conditions and the integration scheme. The upper reference limit for the optimized method was determined to be $1.7 \%$, a value that is in good agreement with the recent HPLC literature $[21,22,42]$ (chapter B.3).

After introduction of the optimized assay into the routine determination of CDT, more than 600 patient sera were analyzed in the first year and the suitability of the method for routine application could thereby be demonstrated. The internal quality control over this time period $(n=62)$ showed the outstanding reproducibility of the assay with RSD values of $3.8 \%$ and $2.2 \%$ for mean $\mathrm{Tf}$ isoform levels of $1.01 \%$ and $4.19 \%$, respectively. Interference free patterns were monitored except for selected sera of patients with severe liver diseases. Tf patterns obtained with rare heterozygous genetic Tf-BC and Tf-CD variants could easily be identified. Furthermore, the nature of unusual non-Tf peaks detected in some sera was clarified, such as the presence of C-reactive protein and monoclonal and biclonal gammopathia. Application of the assay to confirm positive results produced by an immunoassay during long-term monitoring of a patient showed that the CZE method was appropriate for detecting relapse drinking. Apparent elimination half lifes for asialo-, disialo-Tf and CDT were estimated to be 4.9, 7.2 and 6.7 days, respectively (chapter B.4).

The optimized CZE method for the determination of CDT in human serum represents a highly reproducible, fast and reliable assay with simpler sample preparation, easier column regeneration, faster analysis time and higher resolution of $\mathrm{Tf}$ isoforms compared to the most recent and optimized HPLC approach [23]. The CZE-based assay can therefore be considered as an attractive alternative to HPLC and fulfills the requirements of a reference method. It can be employed for screening purposes, longitudinal monitoring of patients during alcohol withdrawal, detection of relapse drinking and confirmation analyses in clinical chemistry and forensic medicine. 


\section{References}

[1] Musshoff, F., Daldrup, Th., J. Chromatogr. B 1998, 713, 245-264.

[2] Tagliaro, F., Bortolotti, F., Crivellente, F., Cittadini, F., Forensic Sci. Rev. 2000, 12, 134-148.

[3] Sharpe, P.C., Ann. Clin. Biochem. 2001, 38, 652-664.

[4] Musshoff, F., J. Chromatogr. B 2002, 781, 457-480.

[5] Ernst, C., Alkoholmissbrauch - Alkoholabhängigkeit - Alkoholismus, ein Leitfaden für Ärzte und andere in der Alkoholismmus-Behandlung tätige Personen, 3. neubearbeitete und erweiterte Auflage 1989, Eidg. Drucksachenund Materialzentrale, Bern

[6] Gutjahr, E., Gmel, G., Die epidemiologischen Grundlagen zur Schätzung der sozialen Kosten des Alkoholismus in der Schweiz, 1995 - 1998, Schweizerische Fachstelle für Alkohol- und andere Drogenprobleme SFA/ISPA, Lausanne, 161174.

[7] www.bag.admin.ch

[8] Stibler, H., Clin. Chem. 1991, 37, 2029-2037.

[9] Stibler, H., Kjellin, K.G., J. Neurol. Sci. 1976, 30, 269-285.

[10] Stibler, H., Allgulander, C., Borg, S., Kjellin, K.G., Acta med. Scand. 1978, 204, 49-56.

[11] Stibler, H., Borg, S., Allgulander, C., Acta med. Scand. 1979, 206, 275-281.

[12] De Jong, G., van Ejik, H.G., Electrophoresis 1988, 9, 589-598.

[13] Van Eijk, H.G., van Noort, W.L., de Jong, G., Koster, J.F., Clin. Chim. Acta 1987, 165, 141-145.

[14] Landberg, E., Påhlsson, P., Lundblad, A., Arnetorp, A., Jeppsson, J.-O., Biochem. Biophys. Res. Commun. 1995, 210, 267-274.

[15] Inoue, T., Yamauchi, M., Ohkawa, K., Electrophoresis 1999, 20, 452-457.

[16] Sillanaukee, P., Strid, N., Allen, J.P., Litten, R.Z., Alcohol. Clin. Exp. Res. 2001, 25, 34-40.

[17] Arndt, T., Clin. Chem. 2001, 47, 13-27.

[18] Stibler, H., Borg, S., Joustra, M., Alcohol. Clin. Exp. Res. 1986, 10, 535-544.

[19] Stibler, H., Borg, S., Joustra, M., Alcohol Alcohol. 1991, 1 (Suppl.), 451-454.

[20] Helander, A., Fors, M., Zakrisson, B., Alcohol Alcohol. 2001, 36, 406-412.

[21] Jeppsson, J.O., Kristensson, H., Fimiani, C., Clin. Chem. 1993, 39, 2115-2120.

[22] Helander, A., Eriksson, G., Stibler, H., Jeppsson, J.-O., Clin. Chem. 2001, 47, 1225-1233.

[23] Helander, A., Husa, A., Jeppsson, J.-O., Clin. Chem. 2003, 49, 1881-1890. 
[24] Thormann, W., Lurie, I.S., McCord, B., Marti, U., Cenni, B., Malik, N., Electrophoresis 2001, 22, 4216-4243.

[25] Thormann, W., Ther. Drug. Monit. 2002, 24, 222-231.

[26] Kašička, V., Electrophoresis 2001, 22, 4139-4162.

[27] Hutterer, K., Dolník, V., Electrophoresis 2003, 24, 3998-4012.

[28] Rodriguez, I., Li, S.F.Y., Analytica Chimica Acta 1999, 383, 1-26.

[29] Horvath, J., Dolník, V., Electrophoresis 2001, 22, 644-655.

[30] Righetti, P.G., Gelfi, C., Verzola, B., Castelletti, L., Electrophoresis 2001, 22, 603-611.

[31] Stathakis, C., Arriaga, E.A., Lewis, D.F., Dovichi, N.J., J. Chromatogr. A 1998, 817, 227-232.

[32] Katayama, H., Ishihama, Y., Asakawa, N., Anal. Sci. 1998, 14, 407-408.

[33] Katayama, H., Ishihama, Y., Asakawa, N., Anal. Chem. 1998, 70, 2254-2260.

[34] Katayama, H., Ishihama, Y., Asakawa, N., Anal. Chem. 1998, 70, 5272-5277.

[35] Bendahl, L., Hansen, S.H., Gammelgaard, B., Electrophoresis 2001, 22, $2565-$ 2573.

[36] Graul, T.W., Schlenoff, J.B., Anal. Chem. 1999, 71, 4007-4013.

[37] Oda, R., Landers, J.P., Electrophoresis 1996, 17, 431-437.

[38] Giordano, B.C., Muza, M., Trout, A., Landers, J.P., J. Cromatogr. B 2000, 742, 79-89.

[39] Tagliaro, F., Bortolotti, F., Zuliani, M., Crivellente, F., Manetto, G., Pascali, V.L., Marigo, M., J. Cap. Elec. 1999, 6, 137-143.

[40] Crivellente, F., Fracasso, G., Valentini, R., Manetto, G., Riviera, A.P., Tagliaro, F., J. Chromatogr. B 2000, 739, 81-93.

[41] Kennedy, J.W., Carey, R.N., Coolen, R.B., Garber, C.C., Lee, H.T., Levine, J.B., Osberg, I.M., Evaluation of Precision Performance of Clinical Chemistry Devices; Approved Guideline. NCCLS document EP5-A, NCCLS, Wayne, PA, USA, 1999.

[42] Turpeinen, U., Methuen, T., Alfthan, H., Laitinen, K., Salaspuro, M., Stenman, U.-H., Clin. Chem. 2001, 47, 1782-1787. 


\section{E. Publications}

Lanz, C., Kuhn, M., Bortolotti, F., Tagliaro, F., Thormann, W. Evaluation and optimization of capillary zone electrophoresis with different dynamic capillary coatings for the determination of carbohydrate-deficient transferrin in human serum. J. Chromatogr. A 2002, 979, $43-57$.

Lanz, C., Marti, U., Thormann, W. Capillary zone electrophoresis with a dynamic double coating for analysis of carbohydrate-deficient transferrin in human serum: Precision performance and pattern recognition. J. Chromatogr. A 2003, 1013, 131 147.

Lanz, C., Thormann, W. Capillary zone electrophoresis with a dynamic double coating for analysis of carbohydrate-deficient transferrin in human serum: Impact of resolution between disialo- and trisialotransferrin on reference limits. Electrophoresis 2003, 24, $4272-4281$.

Lanz, C., Kuhn, M., Deiss, V., Thormann, W. Improved capillary electrophoresis method for the determination of carbohydrate-deficient transferrin in patient sera. Electrophoresis 2004, 25, 2309 - 2318. 